\title{
LiCl-Mediated Preparation of Highly \\ Functionalized Benzylic Zinc Chlorides
}

\author{
Albrecht Metzger, Matthias A. Schade and Paul Knochel* \\ Department of Chemistry and Biochemistry, Ludwig-Maximilians-University Munich, \\ Butenandtstrasse 5-13, Haus F, 81377 Munich (Germany)
}

\section{Supporting Information}

\author{
$\underline{\text { Table of contents }}$ \\ General and Typical Procedures (2 - 4) \\ Experimental Procedures and Analytical Data (4 - 29) \\ NMR Spectra (30 - 60)
}


General All reactions were carried out under an argon atmosphere in dried glassware. All starting materials which were purchased from commercial sources were used without further purification. Solvents were dried according to standard method by distillation over drying agents under nitrogen atmosphere as follows: DCM $\left(\mathrm{CaH}_{2}\right)$, toluene $(\mathrm{Na})$, diethyl ether (Na/benzophenon). THF was continuously refluxed and freshly distilled from sodium benzophenone ketyl under nitrogen before use. Yields refer to isolated yields of compounds estimated to be $>95 \%$ pure as determined by ${ }^{1} \mathrm{H}-\mathrm{NMR}$ and capillary GC.

\section{Preparation of the reagent $\mathrm{CuCN} \cdot 2 \mathrm{LiCl}\left(1 \mathrm{M}\right.$ solution in THF). ${ }^{1}$}

$\mathrm{CuCN} \cdot 2 \mathrm{LiCl}$ was prepared by drying $\mathrm{CuCN}(896 \mathrm{mg}, 10.0 \mathrm{mmol})$ and $\mathrm{LiCl}(848 \mathrm{mg}$, $20.0 \mathrm{mmol}$ ) in a Schlenk-flask under vacuum for $5 \mathrm{~h}$ at $140{ }^{\circ} \mathrm{C}$. After cooling to $25^{\circ} \mathrm{C}$, dry THF $(10 \mathrm{~mL})$ was added and stirring was continued until the salts were dissolved $(24 \mathrm{~h})$.

\section{Preparation of the reagent $\mathrm{ZnCl}_{2}$ (1 M solution in THF).}

A dry and argon flushed $250 \mathrm{~mL}$ Schlenk-flask, equipped with a magnetic stirring bar and a septum, was charged with $\mathrm{ZnCl}_{2}(13.6 \mathrm{~g}, 100 \mathrm{mmol})$. The salt was heated to $140{ }^{\circ} \mathrm{C}$ under high vacuum for $4 \mathrm{~h}$. After cooling to $25^{\circ} \mathrm{C}$, dry THF $(100 \mathrm{~mL})$ was added and stirring was continued until the salt was dissolved $(4 \mathrm{~h})$.

\section{Preparation of the reagent TMPMgCl·LiCl.}

A dry and argon flushed $250 \mathrm{~mL}$ Schlenk-flask, equipped with a magnetic stirring bar and a septum, was charged with freshly titrated $i \mathrm{PrMgCl} \cdot \mathrm{LiCl}^{2}(100 \mathrm{~mL}, 1.2 \mathrm{M}$ in THF, $120 \mathrm{mmol})$. 2,2,6,6-Tetramethylpiperidine (TMPH, $19.8 \mathrm{~g}, 126 \mathrm{mmol}$ ) was added dropwise at $20^{\circ} \mathrm{C}$. The reaction mixture was stirred at $20^{\circ} \mathrm{C}$ until gas evolution was completed (24-48 h).

TP1: Typical Procedure for the preparation of 1-[3-(chloromethyl)phenyl]-substituted ketones (2g-i).

$\mathrm{ZnCl}_{2}$ solution $(18.8 \mathrm{~mL}, 18.8 \mathrm{mmol}, 1 \mathrm{M}$ in $\mathrm{THF})$ was added to the Grignard compound $(18.0 \mathrm{mmol})$ at the required temperature. The solution was stirred for $30 \mathrm{~min}$. $\mathrm{CuCN} \cdot 2 \mathrm{LiCl}$ solution (19.5 mL, $19.5 \mathrm{mmol}, 1 \mathrm{M}$ in THF) was added and the reaction mixure was stirred for additional $30 \mathrm{~min}$. 3-(Chloromethyl)benzoyl chloride $(2.84 \mathrm{~g}, 15.0 \mathrm{mmol})$ was added dropwise and the solution was stirred for $2 \mathrm{~h}$. The reaction mixture was quenched with $60 \mathrm{~mL}$ of a mixture of sat. aqueous $\mathrm{NH}_{4} \mathrm{Cl} / \mathrm{NH}_{3}\left(25 \%\right.$ in $\left.\mathrm{H}_{2} \mathrm{O}\right)=2: 1$. The layers were separated and the organic layer was extracted with $60 \mathrm{~mL}$ of a mixture of sat. aqueous $\mathrm{NH}_{4} \mathrm{Cl} / \mathrm{NH}_{3}(25 \%$ in 
$\left.\mathrm{H}_{2} \mathrm{O}\right)=2: 1$. The combined aqueous layers were extracted with $\mathrm{Et}_{2} \mathrm{O}(3 \times 250 \mathrm{~mL})$. The combined organic extracts were dried over $\mathrm{MgSO}_{4}$. Evaporation of the solvents in vacuo and purification by column chromatography afforded the expected ketones.

TP2: Typical Procedure for the preparation of the benzylic zinc reagents (1a-m).

A Schlenk-flask equipped with a magnetic stirring bar and a septum was charged with $\mathrm{LiCl}$ (1.5-2.0 equiv). The flask was heated with a heat gun $\left(400^{\circ} \mathrm{C}\right)$ for $10 \mathrm{~min}$ under high vacuum. After cooling to $25^{\circ} \mathrm{C}$, the flask was flushed with argon (3 times). Zinc dust ${ }^{3}$ (1.52.0 equiv) was added followed by THF. 1,2-Dibromethane was added ( $5 \mathrm{~mol} \%$ ) and the reaction mixture was heated until ebullition occurs. After cooling to $25^{\circ} \mathrm{C}$, trimethylsilyl chloride $(1 \mathrm{~mol} \%)$ was added and the mixture was heated again until ebullition occurs. The benzylic chloride ( 1.0 equiv) was added at the required temperature (usually $25^{\circ} \mathrm{C}$ ) as a solution in THF (usually $4 \mathrm{M}$ ). When capillary GC analysis of a hydrolyzed aliquot containing an internal standard showed a conversion of $>98 \%$, the Schlenk-flask was centrifuged for $75 \mathrm{~min}$ at $2000 \mathrm{rpm}$ or the reaction mixture was allowed to settle down for some hours. The yield of the resulting benzylic zinc chloride was determined by iodiometric titration. $^{2}$

TP3: Typical Procedure for the reaction of benzylic zinc chlorides with aldehydes (preparation of secondary benzyl alcohols).

The aldehyde (1.0 equiv) was dissolved in $\mathrm{THF}$ at $0{ }^{\circ} \mathrm{C}$ and the benzylic zinc chloride solution (1.3 equiv) was added dropwise. The resulting solution was allowed to warm slowly to $25{ }^{\circ} \mathrm{C}$ and was stirred for the required time. Then, sat. aqueous $\mathrm{NH}_{4} \mathrm{Cl}(20 \mathrm{~mL})$ solution was added. The phases were separated and the aqueous layer was extracted with $\mathrm{Et}_{2} \mathrm{O}$ $(3 \times 20 \mathrm{~mL})$. The combined organic extracts were dried over $\mathrm{MgSO}_{4}$. Evaporation of the solvents in vacuo and purification by flash chromatography afforded the expected alcohols.

\section{TP4: Typical Procedure for the reaction of benzylic zinc chlorides with acid chlorides (preparation of substituted ketone derivatives).}

To a $\mathrm{CuCN} \cdot 2 \mathrm{LiCl}$ solution (1.4 equiv) at $-25{ }^{\circ} \mathrm{C}$ was added dropwise the desired benzylic zinc chloride solution ( 1.4 equiv). The resulting reaction mixture was stirred for $15 \mathrm{~min}$ at this temperature. Then, the solution was cooled to the required temperature and the acid chloride (1.0 equiv) was added dropwise. The reaction mixture was stirred overnight and allowed to warm to $25^{\circ} \mathrm{C}$. Then, a mixture of sat. aqueous $\mathrm{NH}_{4} \mathrm{Cl} / \mathrm{NH}_{3}\left(25 \%\right.$ in $\left.\mathrm{H}_{2} \mathrm{O}\right)=2: 1$ was added, 
the layers were separated and the aqueous layer was extracted with $\mathrm{Et}_{2} \mathrm{O}(3 \times 100 \mathrm{~mL})$. The combined organic extracts were dried over $\mathrm{MgSO}_{4}$. Evaporation of the solvents in vacuo and purification by flash chromatography afforded the expected ketones.

\section{TP5: Typical Procedure for the reaction of benzylic zinc chlorides with unsaturated ketones (preparation of 3-benzylsubstituted cyclic ketones).}

To a $\mathrm{CuCN} \cdot 2 \mathrm{LiCl}$ solution ( 1.25 equiv) at $-25^{\circ} \mathrm{C}$ was added dropwise the desired benzylic zinc chloride solution (1.25 equiv). The resulting reaction mixture was stirred for $15 \mathrm{~min}$ at this temperature. Then, the solution was cooled to the required temperature and a mixture of the unsaturated ketone ( 1.0 equiv), trimethylsilyl chloride ( 2.5 equiv) and THF was added dropwise. The reaction mixture was stirred overnight and allowed to reach $25^{\circ} \mathrm{C}$. Then, a mixture of sat. aqueous $\mathrm{NH}_{4} \mathrm{Cl} / \mathrm{NH}_{3}\left(25 \%\right.$ in $\left.\mathrm{H}_{2} \mathrm{O}\right)=2: 1$ was added. The layers were separated and the aqueous layer was extracted with $\mathrm{Et}_{2} \mathrm{O}(3 \times 100 \mathrm{~mL})$. The combined extracts were dried over $\mathrm{MgSO}_{4}$. Evaporation of the solvents in vacuo and purification by flash chromatography afforded the expected ketones.

\section{Starting materials}

Preparation of benzylic chlorides of type 2.

3-(Chloromethyl)benzonitrile (2f):

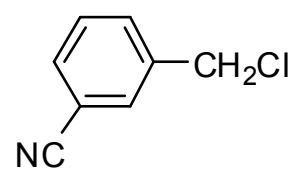

$\mathrm{LiCl}$ (6.36 g, $150 \mathrm{mmol}$ ) was dried (high vacuum, heat gun $400{ }^{\circ} \mathrm{C}, 10 \mathrm{~min}$ ). 3(Bromomethyl)benzonitrile $(9.8 \mathrm{~g}, 50.0 \mathrm{mmol})$ was added followed by THF $(100 \mathrm{~mL})$ at $0{ }^{\circ} \mathrm{C}$. The reaction mixture was refluxed for $5 \mathrm{~h}$. The resulting suspension was transferred into a separation funnel, washed with water $(3 \times 150 \mathrm{~mL})$ and dried over $\mathrm{MgSO}_{4}$ followed by the evaporation of the solvents in vacuo. Again, $\mathrm{LiCl}(6.36 \mathrm{~g}, 150 \mathrm{mmol})$ was dried (high vacuum, heat gun $400{ }^{\circ} \mathrm{C}, 10 \mathrm{~min}$ ) and the crude product was added followed by THF $(100 \mathrm{~mL})$ at $0{ }^{\circ} \mathrm{C}$. The reaction mixture was refluxed for $5 \mathrm{~h}$. The resulting suspension was transferred into a separation funnel, washed with water $(3 \times 150 \mathrm{~mL})$ and dried over $\mathrm{MgSO}_{4}$ followed by the evaporation of the solvents in vacuo. Once again, $\mathrm{LiCl}(6.36 \mathrm{~g}, 150 \mathrm{mmol})$ was dried (high vacuum, heat gun $400{ }^{\circ} \mathrm{C}, 10 \mathrm{~min}$ ) and the crude product was added followed by THF $(100 \mathrm{~mL})$ at $0{ }^{\circ} \mathrm{C}$. The resulting suspension was transferred into a separation funnel, washed with water $(3 \times 150 \mathrm{~mL})$ and dried over $\mathrm{MgSO}_{4}$ followed by the evaporation of the 
solvents in vacuo. Purification by flash chromatography (short column, silica gel, pentane $\left./ \mathrm{Et}_{2} \mathrm{O}=9: 1\right)$ afforded the benzylic chloride $2 \mathbf{f}(7.47 \mathrm{~g}, 99 \%)$ as a white solid. $\mathbf{m p}\left({ }^{\circ} \mathrm{C}\right)=73.3-74.5$.

${ }^{1} \mathbf{H}-\mathbf{N M R}\left(300 \mathrm{MHz}, \mathrm{CDCl}_{3}\right): \delta / \mathrm{ppm}=7.70-7.65(\mathrm{~m}, 1 \mathrm{H}), 7.65-7.57(\mathrm{~m}, 2 \mathrm{H}), 7.52-7.43$ $(\mathrm{m}, 1 \mathrm{H}), 4.58(\mathrm{~s}, 2 \mathrm{H})$.

${ }^{13}$ C-NMR $\left(300 \mathrm{MHz}, \mathrm{CDCl}_{3}\right): \delta / \mathrm{ppm}=138.8,132.8,131.9,131.9,129.6,118.2,112.9$, 44.6.

IR (Diamond-ATR, neat): $\tilde{v} / \mathrm{cm}^{-1}=3060$ (w), 2227 (m), $1584(\mathrm{w}), 1484(\mathrm{~m}), 1445$ (m), 1275 (m), 1240 (m), 1153 (m), 930 (w), 907 (m), 894 (w), 804 (s), 718 (m), 701 (vs), 679 (vs).

MS (EI, $70 \mathrm{eV}): \mathrm{m} / \mathrm{z}(\%)=151$ (100), 117 (17), $116(83), 89$ (45), 63 (11).

HRMS ( $\left.\mathbf{C}_{8} \mathbf{H}_{6} \mathbf{C I N}\right)$ : calc.: 151.0189; found: $151.0183\left(\mathrm{M}^{+}\right)$.

\section{1-[3-(Chloromethyl)phenyl]pentan-1-one (2g):}<smiles>O=C(CBr)c1cccc(CCl)c1</smiles>

According to TP1 butylmagnesium chloride (12.2 mL, 18.0 mmol, 1.48 $\mathrm{M}$ in THF/toluene) was reacted with $\mathrm{ZnCl}_{2}$ solution $(18.8 \mathrm{~mL}, 18.8 \mathrm{mmol}, 1 \mathrm{M}$ in THF), $\mathrm{CuCN} \cdot 2 \mathrm{LiCl}$ solution (19.5 mL, $19.5 \mathrm{mmol}, 1 \mathrm{M}$ in THF) and 3-(chloromethyl)benzoyl chloride (2.84 g, $15.0 \mathrm{mmol}$ ) at $-25^{\circ} \mathrm{C}$. Purification by flash chromatography (silica gel, pentane $/ \mathrm{Et}_{2} \mathrm{O}=2: 1$ ) afforded the benzylic chloride $2 \mathrm{~g}(2.89 \mathrm{~g}, 91 \%)$ as a colourless liquid.

${ }^{1} \mathbf{H}-\mathbf{N M R}\left(300 \mathrm{MHz}, \mathrm{CDCl}_{3}\right): \delta / \mathrm{ppm}=7.97-7.95(\mathrm{~m}, 1 \mathrm{H}), 7.90(\mathrm{dt}, J=7.7 \mathrm{~Hz}, J=1.3 \mathrm{~Hz}$, 1H), 7.60-7.56 (m, 1H), $7.45(\mathrm{t}, J=7.7 \mathrm{~Hz}, 1 \mathrm{H}), 4.62(\mathrm{~s}, 2 \mathrm{H}), 2.96(\mathrm{t}, J=7.1 \mathrm{~Hz}, 2 \mathrm{H}), 1.77-$ $1.66(\mathrm{~m}, 2 \mathrm{H}), 1.47-1.34(\mathrm{~m}, 3 \mathrm{H}), 0.95(\mathrm{t}, J=7.3 \mathrm{~Hz}, 3 \mathrm{H})$.

${ }^{13}$ C-NMR (75 MHz, $\left.\mathrm{CDCl}_{3}\right): \delta / \mathrm{ppm}=200.2,138.3,137.8,133.1,129.3,128.3,128.3,45.9$, $38.7,26.6,22.7,14.2$.

IR (Diamond-ATR, neat): $\widetilde{v} / \mathrm{cm}^{-1}=2957$ (m), 2931 (w), 2871 (w), 1717 (w), 1682 (s), 1443 (w), 1260 (m), 1233 (w), 1199 (w), 1179 (m), 1162 (m), 1109 (w), 1036 (w), 790 (w), 760 (w), $704(\mathrm{vs}), 654(\mathrm{~m})$.

MS (EI, $70 \mathrm{eV}): \mathrm{m} / \mathrm{z}(\%)=210\left(\mathrm{M}^{+}, 6\right), 175$ (13), 170 (19), 168 (54), 155 (33), 154 (12), 153 (100), 125 (25), 89 (18).

HRMS ( $\left.\mathbf{C}_{\mathbf{1 2}} \mathbf{H}_{\mathbf{1 5}} \mathbf{C l O}\right)$ : calc.: 210.0811; found: $210.0798\left(\mathrm{M}^{+}\right)$. 


\section{1-[3-(Chloromethyl)phenyl]propan-1-one (2h):}

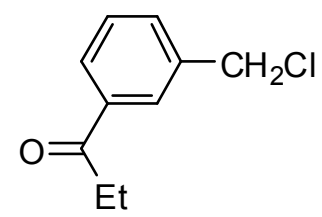

According to TP1 ethylmagnesium bromide (21.2 mL, 18.0 mmol, 0.85 $\mathrm{M}$ in $t$-BuOMe) was reacted with $\mathrm{ZnCl}_{2}$ solution $(18.8 \mathrm{~mL}, 18.8 \mathrm{mmol}, 1 \mathrm{M}$ in THF), $\mathrm{CuCN} \cdot 2 \mathrm{LiCl}$ solution (19.5 mL, $19.5 \mathrm{mmol}, 1 \mathrm{M}$ in THF) and 3-(chloromethyl)benzoyl chloride (2.84 g, $15.0 \mathrm{mmol}$ ) at $-25{ }^{\circ} \mathrm{C}$. Purification by flash chromatography (silica gel, pentane $/ \mathrm{Et}_{2} \mathrm{O}=1: 1$ ) afforded the benzylic chloride $\mathbf{2 h}(2.89 \mathrm{~g}, 94 \%)$ as a colourless liquid.

${ }^{1} \mathbf{H}-\mathbf{N M R}\left(300 \mathrm{MHz}, \mathrm{CDCl}_{3}\right): \delta / \mathrm{ppm}=7.98-7.95(\mathrm{~m}, 1 \mathrm{H}), 7.90(\mathrm{dt}, J=7.7 \mathrm{~Hz}, J=1.3 \mathrm{~Hz}$, 1H), 7.60-7.56 (m, 1H), $7.45(\mathrm{t}, J=7.5 \mathrm{~Hz}, 1 \mathrm{H}), 4.62(\mathrm{~s}, 2 \mathrm{H}), 3.00(\mathrm{t}, J=7.3 \mathrm{~Hz}, 2 \mathrm{H}), 1.22$ (t, $J=7.3 \mathrm{~Hz}, 3 \mathrm{H})$.

${ }^{13}$ C-NMR $\left(75 \mathrm{MHz}, \mathrm{CDCl}_{3}\right): \delta / \mathrm{ppm}=200.5,138.3,137.6,133.1,129.3,128.2,128.2,45.9$, 32.1, 8.4.

IR (Diamond-ATR, neat): $\widetilde{v} / \mathrm{cm}^{-1}=2978$ (w), 1682 (s), 1604 (w), $1586(\mathrm{w}), 1444$ (w), 1378 (w), 1350 (m), 1270 (w), 1242 (s), 1184 (m), 1164 (s), 974 (m), 786 (m), 704 (vs).

MS (EI, $70 \mathrm{eV}): \mathrm{m} / \mathrm{z}(\%)=182\left(\mathrm{M}^{+}, 7\right), 153$ (100), 147 (14), 125 (27), 90 (14), 89 (19), 44 (16).

HRMS ( $\left.\mathbf{C}_{10} \mathbf{H}_{11} \mathbf{C l O}\right)$ : calc.: 182.0498; found: $182.0472\left(\mathrm{M}^{+}\right)$.

\section{1-[3-(Chloromethyl)phenyl]propan-1-one (2i):}<smiles>CC(=O)c1cccc(CCl)c1</smiles>

According to TP1 methylmagnesium chloride (7.03 mL, $18.0 \mathrm{mmol}, 2.56 \mathrm{M}$ in THF) was reacted with $\mathrm{ZnCl}_{2}$ solution $(18.8 \mathrm{~mL}, 18.8 \mathrm{mmol}, 1 \mathrm{M}$ in THF), CuCN.2LiCl solution (19.5 mL, $19.5 \mathrm{mmol}, 1 \mathrm{M}$ in THF) and 3-(chloromethyl)benzoyl chloride (2.84 g, $15.0 \mathrm{mmol}$ ) at $-10{ }^{\circ} \mathrm{C}$. Purification by flash chromatography (silica gel, pentane $/ \mathrm{Et}_{2} \mathrm{O}=3: 1$ ) afforded the benzylic chloride $\mathbf{2 i}(2.46 \mathrm{~g}, 97 \%)$ as a colourless liquid.

${ }^{1} \mathbf{H}-\mathbf{N M R}\left(600 \mathrm{MHz}, \mathrm{C}_{6} \mathrm{D}_{6}\right): \delta / \mathrm{ppm}=7.74-7.71(\mathrm{~m}, 1 \mathrm{H}), 7.58(\mathrm{dt}, J=7.7 \mathrm{~Hz}, J=1.4 \mathrm{~Hz}$, 1H), 7.12-7.04 (m, 1H), 6.93 (t, J=7.7 Hz, 1H), 4.02 (s, 1H), 2.06 (s, 1H). 
${ }^{13}$ C-NMR (150 MHz, $\left.\mathrm{C}_{6} \mathrm{D}_{6}\right): \delta / \mathrm{ppm}=196.0,138.2,137.9,132.8,128.9,128.4,128.2,45.5$, 26.1 .

IR (Diamond-ATR, neat): $\widetilde{v} / \mathrm{cm}^{-1}=1680$ (vs), 1604 (m), 1586 (w), 1440 (m), 1428 (m), 1356 (s), 1280 (s), 1258 (s), 1192 (s), 1174 (m), 976 (w), 956 (w), 798 (m), 702 (vs), 688 (s). MS (EI, $70 \mathrm{eV}): \mathrm{m} / \mathrm{z}(\%)=168\left(\mathrm{M}^{+}, 3\right), 164$ (13), 153 (17), 149 (100), 121 (17), 65 (19), 43 (19).

HRMS (C9H9 $\mathbf{9}$ ClO): calc.: 168.0342; found: $168.0317\left(\mathrm{M}^{+}\right)$.

\section{Preparation of benzyl zinc chlorides of type 1.}

2-Chlorobenzylzinc chloride (1a):

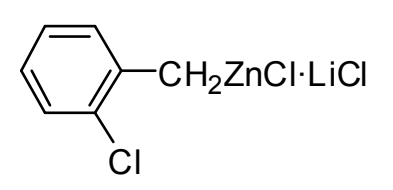

According to TP2 2-chlorobenzyl chloride (2a) (3.22 g, $20.0 \mathrm{mmol}$, in $5 \mathrm{~mL}$ THF) was added dropwise at $0{ }^{\circ} \mathrm{C}$ to a suspension of $\mathrm{LiCl}(1.27 \mathrm{~g}, 30.0 \mathrm{mmol})$ and zinc dust $(1.96 \mathrm{~g}$, $30.0 \mathrm{mmol})$ in $5 \mathrm{~mL}$ THF (activation: $\mathrm{BrCH}_{2} \mathrm{CH}_{2} \mathrm{Br}(0.09 \mathrm{~mL}, 5 \mathrm{~mol} \%)$, TMSCl $(0.03 \mathrm{~mL}$, $1 \mathrm{~mol} \%)$ ). The reaction mixture was stirred for $15 \mathrm{~min}$ at $0{ }^{\circ} \mathrm{C}$ followed by $1.75 \mathrm{~h}$ at $25^{\circ} \mathrm{C}$. After centrifugation iodometric titration ${ }^{2}$ of $1 \mathbf{a}$ indicates a yield of $99 \%$.

\section{4-Fluorobenzylzinc chloride (1b):}<smiles></smiles>

According to TP2 4-fluorobenzyl chloride (2b) (2.17 g, $15.0 \mathrm{mmol}$, in $4 \mathrm{~mL}$ THF) was added dropwise at $25^{\circ} \mathrm{C}$ to a suspension of $\mathrm{LiCl}(1.27 \mathrm{~g}, 30.0 \mathrm{mmol})$ and zinc dust $(1.96 \mathrm{~g}$, $30.0 \mathrm{mmol})$ in $3.5 \mathrm{~mL}$ THF (activation: $\mathrm{BrCH}_{2} \mathrm{CH}_{2} \mathrm{Br}(0.07 \mathrm{~mL}, 5 \mathrm{~mol} \%)$, TMSCl $(0.02 \mathrm{~mL}$, $1 \mathrm{~mol} \%)$ ). The reaction mixture was stirred for $24 \mathrm{~h}$ at $25{ }^{\circ} \mathrm{C}$. After centrifugation iodometric titration $^{2}$ of $\mathbf{1 b}$ indicates a yield of $87 \%$.

\section{3-Bromobenzylzinc chloride (1c):}

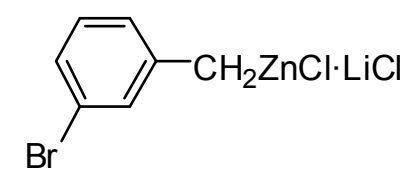

According to TP2 3-bromobenzyl chloride (2c) (4.11 g, $20.0 \mathrm{mmol}$, in $5 \mathrm{~mL}$ THF) was added dropwise at $25^{\circ} \mathrm{C}$ to a suspension of $\mathrm{LiCl}(1.27 \mathrm{~g}, 30.0 \mathrm{mmol})$ and zinc dust $(1.96 \mathrm{~g}$, 
$30.0 \mathrm{mmol})$ in $3.5 \mathrm{~mL}$ THF (activation: $\mathrm{BrCH}_{2} \mathrm{CH}_{2} \mathrm{Br}(0.09 \mathrm{~mL}, 5 \mathrm{~mol} \%)$, TMSCl $(0.03 \mathrm{~mL}$, $1 \mathrm{~mol} \%)$ ). The reaction mixture was stirred for $4 \mathrm{~h}$ at $25^{\circ} \mathrm{C}$. After centrifugation iodometric titration $^{2}$ of $1 \mathrm{c}$ indicates a yield of $95 \%$.

\section{2-Iodobenzylzinc chloride (1d):}

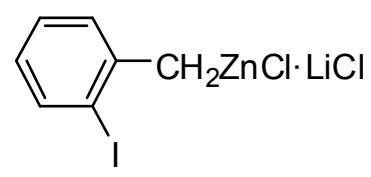

According to TP2 2-iodobenzyl chloride (2d) (5.05 g, $20.0 \mathrm{mmol}$, in $5 \mathrm{~mL}$ THF) was added dropwise at $0{ }^{\circ} \mathrm{C}$ to a suspension of $\mathrm{LiCl}(1.27 \mathrm{~g}, 30.0 \mathrm{mmol})$ and zinc dust $(1.96 \mathrm{~g}$, $30.0 \mathrm{mmol})$ in $5 \mathrm{~mL}$ THF (activation: $\mathrm{BrCH}_{2} \mathrm{CH}_{2} \mathrm{Br}(0.09 \mathrm{~mL}, 5 \mathrm{~mol} \%)$, TMSCl $(0.03 \mathrm{~mL}$, $1 \mathrm{~mol} \%)$ ). The reaction mixture was stirred for $20 \mathrm{~min}$ at $0{ }^{\circ} \mathrm{C}$ followed by $100 \mathrm{~min}$ at $25^{\circ} \mathrm{C}$. After centrifugation iodometric titration ${ }^{2}$ of $\mathbf{1 d}$ indicates a yield of $99 \%$.

\section{3-Ethoxycarbonylbenzylzinc chloride (1e):}

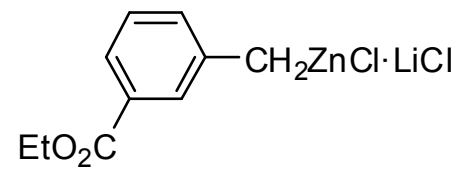

According to TP2 3-ethoxycarbonylbenzyl chloride (2e) (3.97 g, $20.0 \mathrm{mmol}$, in $5 \mathrm{~mL}$ THF) was added dropwise at $25^{\circ} \mathrm{C}$ to a suspension of $\mathrm{LiCl}(1.70 \mathrm{~g}, 40.0 \mathrm{mmol})$ and zinc dust (2.62 g, $40.0 \mathrm{mmol})$ in $5 \mathrm{~mL}$ THF (activation: $\mathrm{BrCH}_{2} \mathrm{CH}_{2} \mathrm{Br}(0.09 \mathrm{~mL}, 5 \mathrm{~mol} \%)$, TMSCl $(0.03 \mathrm{~mL}, 1 \mathrm{~mol} \%))$. The reaction mixture was stirred for $3.5 \mathrm{~h}$ at $25^{\circ} \mathrm{C}$. After centrifugation iodometric titration $^{2}$ of $1 \mathrm{e}$ indicates a yield of $85 \%$.

\section{3-Cyanobenzylzinc chloride (1f):}

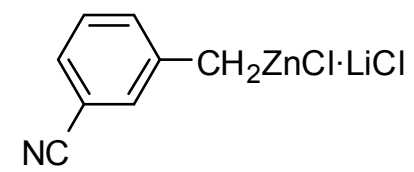

According to TP2 3-cyanobenzyl chloride (2f) (3.03 g, $20.0 \mathrm{mmol}$, in $5 \mathrm{~mL}$ THF) was added dropwise at $0{ }^{\circ} \mathrm{C}$ to a suspension of $\mathrm{LiCl}(1.27 \mathrm{~g}, 30.0 \mathrm{mmol})$ and zinc dust $(1.96 \mathrm{~g}$, $30.0 \mathrm{mmol})$ in $5 \mathrm{~mL}$ THF (activation: $\mathrm{BrCH}_{2} \mathrm{CH}_{2} \mathrm{Br}(0.09 \mathrm{~mL}, 5 \mathrm{~mol} \%)$, TMSCl $(0.03 \mathrm{~mL}$, 
$1 \mathrm{~mol} \%)$ ). The ice bath was removed and the reaction mixture was stirred for $3 \mathrm{~h}$ at $25^{\circ} \mathrm{C}$. After centrifugation iodometric titration ${ }^{2}$ of $\mathbf{1 f}$ indicates a yield of $93 \%$.

\section{3-Pentanoylbenzylzinc chloride (1g):}<smiles></smiles>

According to TP2 3-pentanoylbenzyl chloride (2g) (4.21 g, $20.0 \mathrm{mmol}$, in $5 \mathrm{~mL}$ THF) was added dropwise at $25^{\circ} \mathrm{C}$ to a suspension of $\mathrm{LiCl}(1.27 \mathrm{~g}, 30.0 \mathrm{mmol})$ and zinc dust $(1.96 \mathrm{~g}$, $30 \mathrm{mmol})$ in $5 \mathrm{~mL}$ THF (activation: $\mathrm{BrCH}_{2} \mathrm{CH}_{2} \mathrm{Br}(0.09 \mathrm{~mL}, 5 \mathrm{~mol} \%)$, TMSCl $(0.03 \mathrm{~mL}$, $1 \mathrm{~mol} \%)$ ). The reaction mixture was stirred for $3.5 \mathrm{~h}$ at $25^{\circ} \mathrm{C}$. After centrifugation iodometric titration ${ }^{2}$ of $\mathbf{1 g}$ indicates a yield of $72 \%$.

\section{3-Propionylbenzylzinc chloride (1h):}<smiles></smiles>

According to TP2 3-propionylbenzyl chloride (2h) (2.01 g, $11.0 \mathrm{mmol}$, in $3.5 \mathrm{~mL}$ THF) was added dropwise at $25^{\circ} \mathrm{C}$ to a suspension of $\mathrm{LiCl}(0.70 \mathrm{~g}, 16.5 \mathrm{mmol})$ and zinc dust $(1.08 \mathrm{~g}$, $16.5 \mathrm{mmol})$ in $3 \mathrm{~mL}$ THF (activation: $\mathrm{BrCH}_{2} \mathrm{CH}_{2} \mathrm{Br}(0.05 \mathrm{~mL}, 5 \mathrm{~mol} \%)$, TMSCl $(0.01 \mathrm{~mL}$, $1 \mathrm{~mol} \%)$ ). The reaction mixture was stirred for $3 \mathrm{~h}$ at $25^{\circ} \mathrm{C}$. After centrifugation iodometric titration $^{2}$ of $\mathbf{1 h}$ indicates a yield of $72 \%$.

\section{3-Acetylbenzylzinc chloride (1i):}<smiles></smiles>

According to TP2 3-acetylbenzyl chloride (2il) (1.85 g, $11.0 \mathrm{mmol}$, in $2.5 \mathrm{~mL}$ THF) was added dropwise at $25^{\circ} \mathrm{C}$ to a suspension of $\mathrm{LiCl}(0.70 \mathrm{~g}, 16.5 \mathrm{mmol})$ and zinc dust $(1.08 \mathrm{~g}$, $16.5 \mathrm{mmol})$ in $3 \mathrm{~mL}$ THF (activation: $\mathrm{BrCH}_{2} \mathrm{CH}_{2} \mathrm{Br}(0.05 \mathrm{~mL}, 5 \mathrm{~mol} \%)$, TMSCl (0.01 mL, 
$1 \mathrm{~mol} \%)$ ). The reaction mixture was stirred for $3.5 \mathrm{~h}$ at $25^{\circ} \mathrm{C}$. After centrifugation iodometric titration $^{2}$ of $1 \mathbf{i}$ indicates a yield of $68 \%$.

\section{1-Phenylethylzinc chloride (1j):}

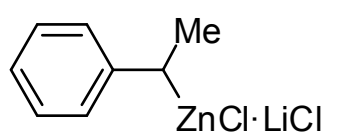

According to TP2 1-phenylethyl chloride (2j) (2.81 g, $20.0 \mathrm{mmol}$, in $5 \mathrm{~mL}$ THF) was added dropwise at $0{ }^{\circ} \mathrm{C}$ to a suspension of $\mathrm{LiCl}(1.27 \mathrm{~g}, 30.0 \mathrm{mmol})$ and zinc dust $(1.96 \mathrm{~g}$, $30.0 \mathrm{mmol})$ in $5 \mathrm{~mL}$ THF (activation: $\mathrm{BrCH}_{2} \mathrm{CH}_{2} \mathrm{Br}(0.09 \mathrm{~mL}, 5 \mathrm{~mol} \%)$, TMSCl $(0.03 \mathrm{~mL}$, $1 \mathrm{~mol} \%)$ ). The ice bath was removed and the reaction mixture was stirred for $11 \mathrm{~h}$ at $25^{\circ} \mathrm{C}$. After centrifugation iodometric titration ${ }^{2}$ of $\mathbf{1 j}$ indicates a yield of $85 \%$.

\section{3,4,5-Trimethoxybenzylzinc chloride (1k):}<smiles>COc1cc(C[C+]Cl)cc(OC)c1OC</smiles>

According to TP2 3,4,5-trimethoxybenzyl chloride (2k) $(2.71 \mathrm{~g}, 12.5 \mathrm{mmol}$, solution in $3 \mathrm{~mL}$ THF) was added dropwise at $0{ }^{\circ} \mathrm{C}$ to a suspension of $\mathrm{LiCl}(1.06 \mathrm{~g}, 25.0 \mathrm{mmol})$ and zinc dust (1.64 g, $25.0 \mathrm{mmol})$ in $3.5 \mathrm{~mL}$ THF (activation: $\mathrm{BrCH}_{2} \mathrm{CH}_{2} \mathrm{Br}(0.05 \mathrm{~mL}, 5 \mathrm{~mol} \%)$, TMSCl $(0.02 \mathrm{~mL}, 1 \mathrm{~mol} \%))$. The ice bath was removed and the reaction mixture was stirred for $3 \mathrm{~h}$ at $25^{\circ} \mathrm{C}$. After centrifugation iodometric titration ${ }^{2}$ of $1 \mathbf{k}$ indicates a yield of $78 \%$.

\section{6-Chloro-1,3-benzodioxol-5-ylmethylzinc chloride (11):}<smiles>Clc1cc2c(cc1CC(Cl)Cl)OCO2</smiles>

According to TP2 6-chloro-1,3-benzodioxol-5-ylmethyl chloride (21) (4.10 g, $20.0 \mathrm{mmol}$, solution in $5 \mathrm{~mL}$ THF) was added dropwise at $0{ }^{\circ} \mathrm{C}$ to a suspension of $\mathrm{LiCl}(1.27 \mathrm{~g}$, $30.0 \mathrm{mmol}$ ) and zinc dust $(1.96 \mathrm{~g}, 30.0 \mathrm{mmol})$ in $5 \mathrm{~mL}$ THF (activation: $\mathrm{BrCH}_{2} \mathrm{CH}_{2} \mathrm{Br}$ $(0.09 \mathrm{~mL}, 5 \mathrm{~mol} \%), \mathrm{TMSCl}(0.03 \mathrm{~mL}, 1 \mathrm{~mol} \%))$. The ice bath was removed and the reaction mixture was stirred for $1 \mathrm{~h}$ at $25^{\circ} \mathrm{C}$. After centrifugation iodometric titration ${ }^{2}$ of 11 indicates a yield of $93 \%$. 
Preparation of the products of type 4 and 5 (reaction of the benzylic zinc reagents with electrophiles).

1-Chloro-2-(cyclohex-2-en-1-ylmethyl)benzene (4a):<smiles>Clc1ccccc1CC1C=CCCC1</smiles>

3-Bromocyclohexene (3a) (419 mg, $2.6 \mathrm{mmol}$ ) was added to 2-chlorobenzylzinc chloride (1a) $(1.23 \mathrm{~mL}, 2.0 \mathrm{mmol}, 1.62 \mathrm{M}$ in $\mathrm{THF})$ at $0^{\circ} \mathrm{C}$, followed by adding $\mathrm{CuCN} \cdot 2 \mathrm{LiCl}$ solution ( 2 drops). The solution was stirred for $1.5 \mathrm{~h}$ at $25^{\circ} \mathrm{C}$. The reaction mixture was quenched with sat. aqueous $\mathrm{NH}_{4} \mathrm{Cl}$ solution. The phases were separated and the aqueous layer was extracted with $\mathrm{Et}_{2} \mathrm{O}(3 \times 5 \mathrm{~mL})$. The combined extracts were dried over $\mathrm{MgSO}_{4}$. Evaporation of the solvents in vacuo and purification by flash chromatography (silica gel, pentane) afforded the cyclohexene 4a (389 mg, 94\%) as a colourless liquid.

${ }^{1} \mathbf{H}-\mathbf{N M R}\left(600 \mathrm{MHz}, \mathrm{CDCl}_{3}\right): \delta / \mathrm{ppm}=7.33(\mathrm{dd}, J=7.7 \mathrm{~Hz}, J=1.3 \mathrm{~Hz}, 1 \mathrm{H}), 7.20-7.11(\mathrm{~m}$, $3 \mathrm{H}), 5.72-5.68(\mathrm{~m}, 1 \mathrm{H}), 5.58-5.54(\mathrm{~m}, 1 \mathrm{H}), 2.77-2.72(\mathrm{~m}, 1 \mathrm{H}), 2.69-2.65(\mathrm{~m}, 1 \mathrm{H}), 2.51-2.43$ (m, 1H), 2.02-1.96 (m, 1H), 1.77-1.66 (m, 2H), 1.55-1.47 (m, 1H), 1.33-1.27 (m, 1H).

${ }^{13}$ C-NMR $\left(150 \mathrm{MHz}, \mathrm{CDCl}_{3}\right): \delta / \mathrm{ppm}=138.5,134.3,131.4,131.0,129.5,127.5,127.3$, 126.4, 40.0, 35.4, 28.8, 25.4, 21.2.

IR (Diamond-ATR, neat): $\widetilde{v} / \mathrm{cm}^{-1}=3017$ (m), 2922 (s), 2857 (m), 2834 (m), 1473 (s), 1446 (m), 1439 (m), 1052 (m), 1032 (m), 746 (vs), 718 (m), 683 (m), 665 (m).

MS (EI, $70 \mathrm{eV}): \mathrm{m} / \mathrm{z}(\%)=208\left(\mathrm{M}^{+}, 9\right), 206$ (31), 125 (22), 82 (12), 81 (24), 80 (100), 79 (24).

HRMS ( $\left.\mathbf{C}_{13} \mathbf{H}_{15} \mathbf{C l}\right)$ : calc.: 206.0862; found: $206.0840\left(\mathrm{M}^{+}\right)$.

\section{1-Chloro-2-[2-(4-nitrophenyl)ethyl]benzene (4b):}<smiles>O=[N+]([O-])c1ccc(CCc2ccccc2Cl)cc1</smiles>

To a solution of 4-nitrobenzyl bromide (3b) $(594 \mathrm{mg}, 2.75 \mathrm{mmol})$ in $2.7 \mathrm{~mL}$ THF at $0{ }^{\circ} \mathrm{C}$ was added successively 2-chlorobenzylzinc chloride (1a) $(2.17 \mathrm{~mL}, 3.3 \mathrm{mmol}, 1.62 \mathrm{M}$ in THF) and $\mathrm{CuCN} \cdot 2 \mathrm{LiCl}$ solution ( 2 drops). The mixture was stirred for $3 \mathrm{~h}$ at $0{ }^{\circ} \mathrm{C}$. The reaction mixture was quenched with sat. aqueous $\mathrm{NH}_{4} \mathrm{Cl}$ solution. The phases were separated and the 
aqueous layer was extracted with $\mathrm{Et}_{2} \mathrm{O}(5 \times 5 \mathrm{~mL})$. The combined extracts were dried over $\mathrm{MgSO}_{4}$. Evaporation of the solvents in vacuo and purification by flash chromatography (silica gel, pentane $\left./ \mathrm{Et}_{2} \mathrm{O}=98: 2\right)$ afforded the diarylethane $\mathbf{4 b}(643 \mathrm{mg}, 89 \%)$ as a white solid. $\mathbf{m p}\left({ }^{\circ} \mathrm{C}\right)=66.9-67.9^{\circ} \mathrm{C}$.

${ }^{1} \mathbf{H}-\mathbf{N M R}\left(600 \mathrm{MHz}, \mathrm{CDCl}_{3}\right): \delta / \mathrm{ppm}=8.14-8.11(\mathrm{~m}, 2 \mathrm{H}), 7.38-7.34(\mathrm{~m}, 1 \mathrm{H}), 7.32-7.29(\mathrm{~m}$, 2H), 7.18-7.13 (m, 2H), 7.09-7.06 (m, 1H), 3.07-3.00 (m, 2H).

${ }^{13}$ C-NMR $\left(150 \mathrm{MHz}, \mathrm{CDCl}_{3}\right): \delta / \mathrm{ppm}=149.3,138.2,134.1,130.7,129.9,129.6,128.1$, $127.1,123.9,36.0,35.4$.

IR (Diamond-ATR, neat): $\widetilde{v} / \mathrm{cm}^{-1}=2932$ (w), 2854 (w), 1596 (m), 1509 (s), 1470 (m), 1457 (m), 1444 (m), 1334 (m), 1313 (m), 1256 (m), 1107 (m), 1049 (m), 1036 (m), 829 (s), 750 (vs), 698 (s).

MS (EI, $70 \mathrm{eV}): \mathrm{m} / \mathrm{z}(\%)=263(11), 261\left(\mathrm{M}^{+}, 29\right), 127$ (33), 125 (100), 89 (13).

HRMS ( $\left.\mathbf{C}_{14} \mathbf{H}_{12} \mathbf{C I N O}_{2}\right)$ : calc.: 261.0557; found: $261.0560\left(\mathrm{M}^{+}\right)$.

\section{Ethyl 4-(2-chlorobenzyl)benzoate (4c):}<smiles>CCOC(=O)c1ccc(Cc2ccccc2Cl)cc1</smiles>

To a solution of ethyl 4-iodobenzoate (3c) $(690 \mathrm{mg}, 2.5 \mathrm{mmol})$ in $2.5 \mathrm{~mL}$ THF at $25{ }^{\circ} \mathrm{C}$ was added successively 2-chlorobenzylzinc chloride (1a) $(1.96 \mathrm{~mL}, 3.0 \mathrm{mmol}, 1.53 \mathrm{M}$ in THF) and $\mathrm{Pd}\left(\mathrm{PPh}_{3}\right)_{4}(69 \mathrm{mg}, 2 \mathrm{~mol} \%)$. The resulting reaction mixture was heated to $60{ }^{\circ} \mathrm{C}$ for $5 \mathrm{~h}$. After cooling to $25^{\circ} \mathrm{C}$, the reaction mixture was diluted with $\mathrm{Et}_{2} \mathrm{O}(5 \mathrm{~mL})$ and quenched with sat. aqueous $\mathrm{NH}_{4} \mathrm{Cl}$ solution. The phases were separated and the aqueous layer was extracted with $\mathrm{Et}_{2} \mathrm{O}(5 \times 5 \mathrm{~mL})$. The combined extracts were dried over $\mathrm{MgSO}_{4}$. Evaporation of the solvents in vacuo and purification by flash chromatography (silica gel, pentane $/ \mathrm{Et}_{2} \mathrm{O}=9: 1$ ) afforded the diarylmethane $4 \mathbf{c}(667 \mathrm{mg}, 97 \%)$ as a pale yellow liquid.

${ }^{1}$ H-NMR $\left(300 \mathrm{MHz}, \mathrm{CDCl}_{3}\right): \delta / \mathrm{ppm}=8.06-8.01(\mathrm{~m}, 3 \mathrm{H}), 7.46-7.42(\mathrm{~m}, 1 \mathrm{H}), 7.34-7.28$ (m, 2H), 7.27-7.18 (m, 2H), $4.42(\mathrm{q}, J=7.2 \mathrm{~Hz}, 2 \mathrm{H}), 4.21(\mathrm{~s}, 2 \mathrm{H}), 1.44(\mathrm{t}, J=7.2 \mathrm{~Hz}, 3 \mathrm{H})$.

${ }^{13}$ C-NMR $\left(75 \mathrm{MHz}, \mathrm{CDCl}_{3}\right): \delta / \mathrm{ppm}=166.8,145.0,138.0,134.5,131.3,130.0,129.9$, $129.1,128.9,128.2,127.2,61.1,39.5,14.6$.

IR (Diamond-ATR, neat): $\tilde{v} / \mathrm{cm}^{-1}=2980(\mathrm{w}), 1712$ (vs), $1610(\mathrm{~m}), 1473(\mathrm{w}), 1443(\mathrm{~m})$, 1415 (m), 1366 (w), 1271 (vs), 1177 (m), 1103 (s), 1050 (m), 1039 (m), 1020 (m), 747 (s). MS (EI, $70 \mathrm{eV}): \mathrm{m} / \mathrm{z}(\%)=276$ (23), 275 (15), $274\left(\mathrm{M}^{+}, 77\right), 248$ (109, 246 (30), 239 (13), 232 (38), 231 (17), 230 (100), 211 (21), 203 (12), 201 (32), 167 (20), 166 (399, 165 (67). 
HRMS ( $\left.\mathbf{C}_{16} \mathbf{H}_{15} \mathbf{C l O}_{2}\right)$ : calc.: 274.0671; found: $274.0748\left(\mathrm{M}^{+}\right)$.

\section{3-(2-Chlorobenzyl)cyclohexanone (4d):}<smiles>O=C1CCCC(Cc2ccccc2Cl)C1</smiles>

According to TP5 a mixture of cyclohex-2-en-1-one (3d) $(480 \mathrm{mg}, 5.0 \mathrm{mmol})$ and TMSCl $(1.6 \mathrm{~mL}, 12.5 \mathrm{mmol})$ in $2 \mathrm{~mL}$ THF was added dropwise to a mixture of $\mathrm{CuCN} \cdot 2 \mathrm{LiCl}(6.3 \mathrm{~mL}$, $6.3 \mathrm{mmol}$ ) and 2-chlorobenzylzinc chloride (1a) (3.83 mL, $6.25 \mathrm{mmol}, 1.63 \mathrm{M}$ in THF) at $-40{ }^{\circ} \mathrm{C}$. The reaction mixture was allowed to reach $25^{\circ} \mathrm{C}$ overnight and was quenched with a mixture of sat. aqueous $\mathrm{NH}_{4} \mathrm{Cl} / \mathrm{NH}_{3}\left(25 \%\right.$ in $\left.\mathrm{H}_{2} \mathrm{O}\right)=2: 1(20 \mathrm{~mL})$. Purification by flash chromatography (silica gel, pentane $/ \mathrm{Et}_{2} \mathrm{O}=4: 1$ ) afforded the ketone $\mathbf{4 d}(1.03 \mathrm{~g}, 93 \%)$ as a colourless liquid.

${ }^{1} \mathbf{H}-\mathbf{N M R}\left(300 \mathrm{MHz}, \mathrm{CDCl}_{3}\right): \delta / \mathrm{ppm}=7.33-7.27(\mathrm{~m}, 1 \mathrm{H}), 7.18-7.05(\mathrm{~m}, 3 \mathrm{H}), 2.81-2.62$ (m, 2H), 2.38-1.94 (m, 6H), 1.89-1.78 (m, 1H), 1.66-1.48 (m, 1H), 1.47-1.32 (m, 1H).

${ }^{13}$ C-NMR $\left(75 \mathrm{MHz}, \mathrm{CDCl}_{3}\right): \delta / \mathrm{ppm}=211.4,137.5,134.4,131.5,129.9,128.0,126.9,47.9$, 41.6, 40.6, 39.6, 31.2, 25.3.

IR (Diamond-ATR, neat): $\widetilde{v} / \mathrm{cm}^{-1}=2936$ (w), 2864 (w), 1708 (vs), 1476 (m), 1444 (m), $1348(\mathrm{w}), 1312(\mathrm{w}), 1224(\mathrm{~m}), 1128(\mathrm{w}), 1052$ (m), 1036 (m), 748 (vs), 680 (s), $596(\mathrm{w})$.

MS (EI, $70 \mathrm{eV}): \mathrm{m} / \mathrm{z}(\%)=222\left(\mathrm{M}^{+}, 3\right), 187$ (39), 186 (23), 164 (18), 142 (19), 130 (10), 129 (24), 127 (11), 125 (28), 115 (16), 97 (87), 91 (29), 89 (14), 69 (100), 55 (46), 44 (15), 41 (58).

HRMS ( $\left.\mathbf{C}_{\mathbf{1 3}} \mathbf{H}_{\mathbf{1 5}} \mathbf{C l O}\right)$ : calc.: 222.0811; found: $222.0800\left(\mathrm{M}^{+}\right)$.

\section{1-(4-Fluorophenyl)-3,3-dimethylbutan-2-one (4e):}<smiles>CC(C)(C)CC(=O)Cc1ccc(F)cc1</smiles>

According to TP4 3,3-dimethylbutyryl chloride (3e) (377 mg, $2.8 \mathrm{mmol}$ ) was added dropwise to a mixture of $\mathrm{CuCN} \cdot 2 \mathrm{LiCl}(3.92 \mathrm{~mL}, 3.9 \mathrm{mmol})$ and 4-fluorobenzylzinc chloride (1b) (2.69 $\mathrm{mL}, 3.9 \mathrm{mmol}, 1.46 \mathrm{M}$ in $\mathrm{THF}$ ) at $-40^{\circ} \mathrm{C}$. The reaction mixture was allowed to reach $25{ }^{\circ} \mathrm{C}$ overnight and was quenched with a mixture of sat. aqueous $\mathrm{NH}_{4} \mathrm{Cl} / \mathrm{NH}_{3}(25 \%$ in $\left.\mathrm{H}_{2} \mathrm{O}\right)=4: 1\left(25 \mathrm{~mL}\right.$ ). Purification by flash chromatography (silica gel, pentane $/ \mathrm{Et}_{2} \mathrm{O}=98: 2$ ) afforded the ketone 4 e (555 $\mathrm{mg}, 95 \%)$ as a pale yellow liquid. 
${ }^{1} \mathbf{H}-\mathrm{NMR}\left(600 \mathrm{MHz}, \mathrm{CDCl}_{3}\right): \delta / \mathrm{ppm}=7.15-7.10(\mathrm{~m}, 2 \mathrm{H}), 7.02-6.97(\mathrm{~m}, 2 \mathrm{H}), 3.36(\mathrm{~s}, 1 \mathrm{H})$, $2.35(\mathrm{~s}, 1 \mathrm{H}), 1.00(\mathrm{~s}, 9 \mathrm{H})$.

${ }^{13}$ C-NMR $\left(150 \mathrm{MHz}, \mathrm{CDCl}_{3}\right): \delta / \mathrm{ppm}=207.8,162.9,161.3,131.2,131.2,130.1,130.1$, 115.7, 115.6, 54.4, 51.2, 31.3, 29.9.

IR (Diamond-ATR, neat): $\widetilde{v} / \mathrm{cm}^{-1}=2956$ (m), 1712 (s), 1508 (vs), 1364 (m), 1352 (m), 1220 (vs), 1160 (m), 1084 (m), 1064 (m), 824 (m), 780 (m), 524 (m).

MS (EI, $70 \mathrm{eV}): \mathrm{m} / \mathrm{z}(\%)=208$ (3), 109 (53), 99 (60), 71 (17), 57 (100), 43 (13), 42 (16).

HRMS ( $\left.\mathbf{C}_{\mathbf{1 3}} \mathbf{H}_{\mathbf{1 7}} \mathbf{F O}\right)$ : calc.: 208.1263; found: $208.1261\left(\mathrm{M}^{+}\right)$.

\section{2-(3-Bromophenyl)-1-(3,4-dichlorophenyl)ethanol (4f):}<smiles>OC(Cc1cccc(Br)c1)c1ccc(Cl)c(Cl)c1</smiles>

According to TP3 3-bromobenzylzinc chloride (1c) (1.72 mL, $2.7 \mathrm{mmol}, 1.56 \mathrm{M}$ in THF) was reacted with 3,4-dichlorobenzaldehyde (3f) $(361 \mathrm{mg}, 2.1 \mathrm{mmol}$, in $1.5 \mathrm{~mL}$ THF). After $17 \mathrm{~h}$ the reaction mixture was quenched with sat. aqueous $\mathrm{NH}_{4} \mathrm{Cl}$ solution. Purification by flash chromatography (silica gel, pentane / $\left.\mathrm{Et}_{2} \mathrm{O}=98: 2\right)$ afforded the alcohol $\mathbf{4 f}(699 \mathrm{mg}, 98 \%)$ as a white solid.

$\mathbf{m p}\left({ }^{\circ} \mathrm{C}\right)=64.2-65.4$.

${ }^{1} \mathbf{H}-\mathbf{N M R}\left(600 \mathrm{MHz}, \mathrm{CDCl}_{3}\right): \delta / \mathrm{ppm}=7.43(\mathrm{~d}, J=2.0 \mathrm{~Hz}, 1 \mathrm{H}), 7.41-7.34(\mathrm{~m}, 3 \mathrm{H}), 7.16(\mathrm{t}$, $J=7.7 \mathrm{~Hz}, 1 \mathrm{H}), 7.12(\mathrm{dd}, J=8.4 \mathrm{~Hz}, J=2.0 \mathrm{~Hz}, 1 \mathrm{H}), 7.06(\mathrm{~d}, J=7.5 \mathrm{~Hz}, 1 \mathrm{H}), 4.81$ (dd, $J=8.4 \mathrm{~Hz}, J=4.6 \mathrm{~Hz}, 1 \mathrm{H}), 2.96-2.85(\mathrm{~m}, 2 \mathrm{H}), 2.09$ (s, 1H).

${ }^{13}$ C-NMR $\left(150 \mathrm{MHz}, \mathrm{CDCl}_{3}\right): \delta / \mathrm{ppm}=143.6,139.6,132.6,132.4,131.5,130.4,130.1$, 130.0, 128.1, 127.8, 125.1, 122.6, 73.8, 45.4.

IR (Diamond-ATR, neat): $\widetilde{v} / \mathrm{cm}^{-1}=3288(\mathrm{~m}), 1564(\mathrm{~m}), 1470(\mathrm{~s}), 1424(\mathrm{~m}), 1202(\mathrm{~m})$, 1128 (m), 1070 (s), 1046 (s), 1026 (s), 998 (s), 884 (s), 782 (vs), 668 (vs).

MS (ESI): m/z (\%) = 328 (3), 175 (100), 111 (40), 91 (20), 75 (10).

HRMS ( $\left.\mathbf{C}_{15} \mathbf{H}_{12} \mathbf{B r C l}_{2} \mathbf{O}_{3}\right)$ : calc.: 388.9352 ; found: $388.9360\left(\mathrm{M}^{+}+\right.$formiate $)$.

1-(3-Chlorophenyl)-2-(2-iodophenyl)ethanol (4g):<smiles>OC(Cc1ccccc1I)c1cccc(Cl)c1</smiles> 
According to TP3 2-iodobenzylzinc chloride (1d) (1.28 mL, 2.0 mmol, 1.53 M in THF) was reacted with 3-chlorobenzaldehyde $(\mathbf{3 g})(211 \mathrm{mg}, 1.5 \mathrm{mmol}$, in $1.5 \mathrm{~mL}$ THF). After $5 \mathrm{~h}$ the reaction mixture was quenched with sat. aqueous $\mathrm{NH}_{4} \mathrm{Cl}$ solution. Purification by flash chromatography (silica gel, pentane $/ \mathrm{Et}_{2} \mathrm{O}=9: 1$ to $7: 3$ ) afforded the alcohol $\mathbf{4 g}$ (470 mg, $87 \%)$ as a pale yellow solid.

$\mathbf{m p}\left({ }^{\circ} \mathrm{C}\right)=67.6-69.5$.

${ }^{1} \mathbf{H}-\mathbf{N M R}\left(600 \mathrm{MHz}, \mathrm{CDCl}_{3}\right): \delta / \mathrm{ppm}=7.86(\mathrm{dd}, J=7.8 \mathrm{~Hz}, J=1.2 \mathrm{~Hz}, 1 \mathrm{H}), 7.45(\mathrm{~m}, 1 \mathrm{H})$, 7.30-7.24 (m, 3H), 7.18 (dd, $J=7.5 \mathrm{~Hz}, J=1.8 \mathrm{~Hz}, 1 \mathrm{H}), 5.01-4.97$ (m, 1H), 3.17-3.13 (m $1 \mathrm{H}), 3.08-3.03(\mathrm{~m}, 1 \mathrm{H}), 1.92(\mathrm{~d}, J=3.3 \mathrm{~Hz}, 1 \mathrm{H})$.

${ }^{13}$ C-NMR $\left(150 \mathrm{MHz}, \mathrm{CDCl}_{3}\right): \delta / \mathrm{ppm}=145.7,140.4,139.7,134.4,131.3,129.7,128.7$, $128.3,127.8,125.9,123.9,100.9,72.8,50.4$.

IR (Diamond-ATR, neat): $\widetilde{v} / \mathrm{cm}^{-1}=3322$ (w), 3252 (w), 1596 (w), 1575 (w), 1468 (m), 1435 (m), 1198 (m), 1055 (s), 1015 (s), 884 (m), 783 (s), 746 (s), 725 (s), 695 (vs).

MS (EI, $70 \mathrm{eV}): \mathrm{m} / \mathrm{z}(\%)=358\left(\mathrm{M}^{+}, 1\right), 218$ (100), 142 (8), 141 (27), 77 (13).

HRMS ( $\left.\mathbf{C}_{14} \mathbf{H}_{12} \mathbf{C I I O}\right)$ : calc.: 357.9621 ; found: $357.9629\left(\mathrm{M}^{+}\right)$.

\section{3-(2-Iodobenzyl)cyclohexanone (4h):}

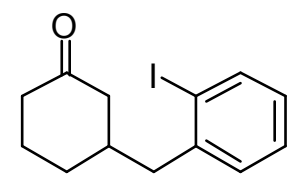

According to TP5 a mixture of cyclohex-2-en-1-one (3d) $(480 \mathrm{mg}, 5.0 \mathrm{mmol})$ and TMSCl $(1.6 \mathrm{~mL}, 12.5 \mathrm{mmol})$ in $2 \mathrm{~mL}$ THF was added dropwise to a mixture of $\mathrm{CuCN} \cdot 2 \mathrm{LiCl}(6.3 \mathrm{~mL}$, $6.3 \mathrm{mmol}$ ) and 2-iodobenzylzinc chloride (1d) (4.81 mL, 6.25 mmol, 1.30 M in THF) at $-40^{\circ} \mathrm{C}$. The reaction mixture was allowed to reach $25^{\circ} \mathrm{C}$ overnight and was quenched with a mixture of sat. aqueous $\mathrm{NH}_{4} \mathrm{Cl} / \mathrm{NH}_{3}\left(25 \%\right.$ in $\left.\mathrm{H}_{2} \mathrm{O}\right)=2: 1(20 \mathrm{~mL})$. Purification by flash chromatography (silica gel, pentane / $\mathrm{Et}_{2} \mathrm{O}=4: 1$ ) afforded the ketone $\mathbf{4 d}(1.13 \mathrm{~g}, 72 \%)$ as a colourless liquid.

${ }^{1} \mathbf{H}-\mathbf{N M R}\left(300 \mathrm{MHz}, \mathrm{CDCl}_{3}\right): \delta / \mathrm{ppm}=7.80(\mathrm{dd}, J=7.8 \mathrm{~Hz}, J=1.2 \mathrm{~Hz}, 1 \mathrm{H}), 7.28-7.22(\mathrm{~m}$, 1H), 7.13-7.09 (m, 1H), 6.91-6.85 (m, 1H), 2.82-2.64 (m, 2H), 2.43-1.98 (m, 6H), 1.95-1.83 (m, 1H), 1.70-1.53 (m, 1H), 1.53-1.37 (m, 1H).

${ }^{13}$ C-NMR $\left(75 \mathrm{MHz}, \mathrm{CDCl}_{3}\right): \delta / \mathrm{ppm}=211.3,142.2,139.7,130.4,128.1$ (overlap), 101.0, $47.6,47.2,41.4,39.5,30.9,25.1$. 
IR (Diamond-ATR, neat): $\widetilde{v} / \mathrm{cm}^{-1}=2933$ (m), 2863 (m), 1706 (vs), 1466 (m), 1446 (m), $1224(\mathrm{~m}), 1008$ (s), 744 (s), $646(\mathrm{~m})$.

MS (EI, $70 \mathrm{eV}): \mathrm{m} / \mathrm{z}(\%)=314\left(\mathrm{M}^{+}, 9\right), 217$ 818), 188 (13), 187 (100), 1269 (15), 115 (16), 97 (66), 91 (22), 89 (12), 69 (72), 55 (34), 41 (33).

HRMS ( $\left.\mathbf{C}_{13} \mathbf{H}_{15} \mathbf{I O}\right)$ : calc.: 314.0168 ; found: $314.0166\left(\mathrm{M}^{+}\right)$.

Ethyl 3-[2-(4-bromophenyl)-2-hydroxyethyl]benzoate (4i):<smiles>CCOC(=O)c1cccc(CC(O)c2ccc(Br)cc2)c1</smiles>

According to TP3 3-ethoxycarbonylbenzylzinc chloride (1e) $(4.1 \mathrm{~mL}, 5.4 \mathrm{mmol}, 1.3 \mathrm{M}$ in THF) was reacted with 4-bromobenzaldehyde (3h) (775 mg, $4.2 \mathrm{mmol}$, in $3 \mathrm{~mL}$ THF). After $4.5 \mathrm{~h}$ the reaction mixture was quenched with sat. aqueous $\mathrm{NH}_{4} \mathrm{Cl}$ solution. Purification by flash chromatography (silica gel, pentane $\left./ \mathrm{Et}_{2} \mathrm{O}=7: 3\right)$ afforded the alcohol $\mathbf{4 i}(1.33 \mathrm{~g}, 91 \%)$ as a white solid.

$\operatorname{mp}\left({ }^{\circ} \mathrm{C}\right)=64.5-65.8$.

${ }^{1}$ H-NMR $\left(600 \mathrm{MHz}, \mathrm{CDCl}_{3}\right): \delta / \mathrm{ppm}=7.92-7.90(\mathrm{~m}, 1 \mathrm{H}), 7.86-7.85(\mathrm{~m}, 1 \mathrm{H}), 7.47-7.44$ (m, 2H), 7.37-7.30 (m, 2H), 7.22-7.19 (m, 2H), 4.91-4.87 (m, 1H), 4.36 (q, $J=7.1 \mathrm{~Hz}, 2 \mathrm{H})$, 3.04-3.01 (m, 2H), $1.97(\mathrm{~d}, J=3.1 \mathrm{~Hz}, 1 \mathrm{H}), 1.39(\mathrm{t}, J=7.2 \mathrm{~Hz}, 3 \mathrm{H})$.

${ }^{13}$ C-NMR $\left(150 \mathrm{MHz}, \mathrm{CDCl}_{3}\right): \delta / \mathrm{ppm}=166.5,142.5,137.9,134.1,131.5,130.7,130.4$, $128.5,127.9,127.6,121.5,74.6,61.0,45.6,14.3$.

IR (Diamond-ATR, neat): $\widetilde{v} / \mathrm{cm}^{-1}=3466$ (w), 1704 (s), 1682 (s), 1484 (m), 1446 (m), 1400 (m), 1366 (m), 1278 (s), 1200 (s), 1108 (s), 1066 (s), 1024 (s), 1004 (s), 746 (vs), 698 (s).

MS (EI, $70 \mathrm{eV}): \mathrm{m} / \mathrm{z}(\%)=348\left(\mathrm{M}^{+},<1\right), 164$ (100), 136 (29), 135 (13), 118 (10), 92 (10), 91 (16), 90 (11), 78 (10), 77 (20).

HRMS ( $\left.\mathbf{C}_{17} \mathbf{H}_{17} \mathrm{BrO}_{3}\right)$ : calc.: 348.0361; found: $348.0372\left(\mathrm{M}^{+}\right)$.

Ethyl 3-[(3-oxocyclohexyl)methyl]benzoate (4j):<smiles>CCOC(=O)c1cccc(CC2CCCC(=O)C2)c1</smiles> 
According to TP5 a mixture of cyclohex-2-en-1-one (3d) (480 mg, $5.0 \mathrm{mmol})$ and TMSCl $(1.6 \mathrm{~mL}, 12.5 \mathrm{mmol})$ in $2 \mathrm{~mL}$ THF was added dropwise to a mixture of $\mathrm{CuCN} \cdot 2 \mathrm{LiCl}(6.3 \mathrm{~mL}$, $6.3 \mathrm{mmol})$ and 3-ethoxycarbonylbenzylzinc chloride (1e) $(4.46 \mathrm{~mL}, 6.25 \mathrm{mmol}, 1.40 \mathrm{M}$ in THF) at $-40{ }^{\circ} \mathrm{C}$. The reaction mixture was allowed to reach $25^{\circ} \mathrm{C}$ overnight and was quenched with a mixture of sat. aqueous $\mathrm{NH}_{4} \mathrm{Cl} / \mathrm{NH}_{3}\left(25 \%\right.$ in $\left.\mathrm{H}_{2} \mathrm{O}\right)=2: 1(20 \mathrm{~mL})$. Purification by flash chromatography (silica gel, pentane $/ \mathrm{Et}_{2} \mathrm{O}=5: 1$ to $1: 1$ ) afforded the ketone $4 \mathbf{j}(1.26 \mathrm{~g}, 97 \%)$ as a colourless liquid.

${ }^{1} \mathbf{H}-\mathbf{N M R}\left(300 \mathrm{MHz}, \mathrm{CDCl}_{3}\right): \delta / \mathrm{ppm}=7.82-7.78(\mathrm{~m}, 1 \mathrm{H}), 7.74-7.72(\mathrm{~m}, 1 \mathrm{H}), 7.28-7.24$ $(\mathrm{m}, 1 \mathrm{H}), 7.24-7.21(\mathrm{~m}, 1 \mathrm{H}), 4.28(\mathrm{q}, J=7.2 \mathrm{~Hz}, 2 \mathrm{H}), 2.63-2.53(\mathrm{~m}, 2 \mathrm{H}), 2.28-2.21(\mathrm{~m}, 2 \mathrm{H})$, $2.20-2.13(\mathrm{~m}, 1 \mathrm{H}), 2.01-1.89(\mathrm{~m}, 3 \mathrm{H}), 1.79-1.73(\mathrm{~m}, 1 \mathrm{H}), 1.57-1.47(\mathrm{~m}, 1 \mathrm{H}), 1.33-1.25(\mathrm{~m}$, $1 \mathrm{H}), 1.30(\mathrm{t}, J=7.2 \mathrm{~Hz}, 3 \mathrm{H})$.

${ }^{13}$ C-NMR $\left(75 \mathrm{MHz}, \mathrm{CDCl}_{3}\right): \delta / \mathrm{ppm}=211.2,166.7,140.0,133.7,130.8,130.2,128.6$, $127.7,61.1,47.8,42.9,41.5,40.9,31.0,25.2,14.5$.

IR (Diamond-ATR, neat): $\widetilde{v} / \mathrm{cm}^{-1}=2936$ (w), 1708 (vs), 1444 (m), 1368 (w), 1276 (vs), $1196(\mathrm{~s}), 1108$ (s), 1024 (m), $864(\mathrm{w}), 748$ (s), 700 (m), $672(\mathrm{w})$.

MS (EI, $70 \mathrm{eV}): \mathrm{m} / \mathrm{z}(\%)=260\left(\mathrm{M}^{+}, 30\right), 215$ (36), 214 (79), 164 (26), 129 (39), 121 (83), 115 (20), 97 (80), 91 (33), 69 (100), 55 (46), 41 (50).

HRMS $\left(\mathbf{C}_{16} \mathbf{H}_{20} \mathbf{O}_{3}\right)$ : calc.: 260.1412; found: $260.1386\left(\mathrm{M}^{+}\right)$.

\section{3-(3-Methoxybenzyl)benzonitrile (4k):}<smiles>COc1cccc(Cc2cccc(C#N)c2)c1</smiles>

To a solution of 3-iodoanisole $(585 \mathrm{mg}, 2.5 \mathrm{mmol})$ in $2.0 \mathrm{~mL}$ THF at $25^{\circ} \mathrm{C}$ was added successively 3-cyanobenzylzinc chloride (1f) $(2.03 \mathrm{~mL}, 3.0 \mathrm{mmol}, 1.48 \mathrm{M}$ in THF) and $\mathrm{Pd}\left(\mathrm{PPh}_{3}\right)_{4}(139 \mathrm{mg}, 5 \mathrm{~mol} \%)$. The resulting reaction mixture was heated to $60{ }^{\circ} \mathrm{C}$ for $5 \mathrm{~h}$. After cooling to $25^{\circ} \mathrm{C}$, the reaction mixture was diluted with $\mathrm{Et}_{2} \mathrm{O}(5 \mathrm{~mL})$ and quenched with sat. aqueous $\mathrm{NH}_{4} \mathrm{Cl}$ solution. The phases were separated and the aqueous layer was extracted with $\mathrm{Et}_{2} \mathrm{O}(5 \times 5 \mathrm{~mL})$. The combined extracts were dried over $\mathrm{MgSO}_{4}$. Evaporation of the solvents in vacuo and purification by flash chromatography (silica gel, pentane $/ \mathrm{Et}_{2} \mathrm{O}=9: 1$ ) afforded the diarylmethane $\mathbf{4 k}(492 \mathrm{mg}, 88 \%)$ as a colourless liquid.

${ }^{1} \mathbf{H}-\mathbf{N M R}\left(300 \mathrm{MHz}, \mathrm{CDCl}_{3}\right): \delta / \mathrm{ppm}=7.51-7.34(\mathrm{~m}, 4 \mathrm{H}), 7.26-7.20(\mathrm{~m}, 1 \mathrm{H}), 6.81-6.67$ (m, 3H), 3.97 (s, 2H), 3.78 (s, 3H). 
${ }^{13}$ C-NMR $\left(75 \mathrm{MHz}, \mathrm{CDCl}_{3}\right): \delta / \mathrm{ppm}=160.2,142.7,141.2,133.6,132.6,130.2,130.0$, $129.5,121.5,119.2,115.2,112.8,112.0,55.4,41.6$.

IR (Diamond-ATR, neat): $\widetilde{v} / \mathrm{cm}^{-1}=2937$ (w), 2228 (s), 1596 (s), 1582 (s), 1488 (s), 1453 (m), 1435 (m), 1257 (vs), 1151 (m), 1048 (s), 779 (m), 741 (m), $686(\mathrm{~s})$.

MS (EI, $70 \mathrm{eV}): \mathrm{m} / \mathrm{z}(\%)=224$ (15), $223\left(\mathrm{M}^{+}, 100\right), 222$ 812), 208 (13), 190 (10).

HRMS ( $\left.\mathbf{C}_{15} \mathbf{H}_{13} \mathbf{N O}\right)$ : calc.: 223.0997; found: $223.0988\left(\mathrm{M}^{+}\right)$.

\section{3-(3,3-Dimethyl-2-oxobutyl)benzonitrile (41):}<smiles>CC(C)(C)C(=O)Cc1cccc(C#N)c1</smiles>

According to TP4 2,3-dimethylpropionyl chloride (3j) (225 mg, $1.87 \mathrm{mmol}$ ) was added dropwise to a mixture of $\mathrm{CuCN} \cdot 2 \mathrm{LiCl}(2.6 \mathrm{~mL}, 2.6 \mathrm{mmol})$ and 3-cyanobenzylzinc chloride (1f) $(1.9 \mathrm{~mL}, 2.6 \mathrm{mmol}, 1.37 \mathrm{M}$ in $\mathrm{THF})$ at $-60{ }^{\circ} \mathrm{C}$. The reaction mixture was allowed to reach $-20{ }^{\circ} \mathrm{C}$ overnight and was quenched with a mixture of sat. aqueous $\mathrm{NH}_{4} \mathrm{Cl} / \mathrm{NH}_{3}(25 \%$ in $\left.\mathrm{H}_{2} \mathrm{O}\right)=5: 1(25 \mathrm{~mL})$. Purification by flash chromatography (silica gel, pentane $/ \mathrm{Et}_{2} \mathrm{O}=6: 1$ ) afforded the ketone $\mathbf{4 l}$ (292 $\mathrm{mg}, 78 \%)$ as a white solid.

$\mathbf{m p}\left({ }^{\circ} \mathrm{C}\right)=38.5-39.7$.

${ }^{1} \mathbf{H}-\mathbf{N M R}\left(600 \mathrm{MHz}, \mathrm{C}_{6} \mathrm{D}_{6}\right): \delta / \mathrm{ppm}=7.03-7.01(\mathrm{~m}, 1 \mathrm{H}), 6.98-6.95(\mathrm{~m}, 2 \mathrm{H}), 6.74(\mathrm{t}$, $J=7.8 \mathrm{~Hz}, 1 \mathrm{H}), 3.13$ (s, 2H), 0.89 (s, 9H).

${ }^{13}$ C-NMR (150 MHz, $\left.\mathrm{C}_{6} \mathrm{D}_{6}\right): \delta / \mathrm{ppm}=209.7,136.7,134.0,133.2,130.2,128.8,118.9,112.9$, $44.3,42.2,26.1$.

IR (Diamond-ATR, neat): $\widetilde{v} / \mathrm{cm}^{-1}=2956(\mathrm{~m}), 2226(\mathrm{~m}), 1700(\mathrm{~s}), 1482(\mathrm{~m}), 1364(\mathrm{~m})$, 1330 (s), 1058 (vs), 1020 (s), 808 (m), 770 (vs), 684 (vs).

MS (EI, $70 \mathrm{eV}): \mathrm{m} / \mathrm{z}(\%)=201\left(\mathrm{M}^{+},<1\right), 117(28), 116(22), 85$ (22), 57 (100), 41 (30).

HRMS ( $\left.\mathbf{C}_{13} \mathbf{H}_{15} \mathbf{N O}\right)$ : calc.: 201.1154; found: $201.1131\left(\mathrm{M}^{+}\right)$.

\section{1-[3-(2-Oxo-2-phenylethyl)phenyl]pentan-1-one (4m):}<smiles>O=C(CBr)c1cccc(CC(=O)c2ccccc2)c1</smiles>

According to TP4 benzoyl chloride (3k) $(278 \mathrm{mg}, 1.98 \mathrm{mmol})$ was added dropwise to a mixture of $\mathrm{CuCN} \cdot 2 \mathrm{LiCl}(2.6 \mathrm{~mL}, 3.9 \mathrm{mmol})$ and 3-pentanoylbenzylzinc chloride (1g) (2.3 $\mathrm{mL}, 2.6 \mathrm{mmol}, 1.15 \mathrm{M}$ in THF) at $-20^{\circ} \mathrm{C}$. The reaction mixture was stirred overnight at 
this temperature followed by quenching with a mixture of sat. aqueous $\mathrm{NH}_{4} \mathrm{Cl} / \mathrm{NH}_{3}(25 \%$ in $\mathrm{H} 2 \mathrm{O})=5: 1(25 \mathrm{~mL})$. Purification by flash chromatography (silica gel, pentane $/ \mathrm{Et}_{2} \mathrm{O}=9: 1$ ) afforded the ketone $\mathbf{4 m}(470 \mathrm{mg}, 85 \%)$ as a white solid.

$\mathbf{m p}\left({ }^{\circ} \mathrm{C}\right)=33.3-36.0$.

${ }^{1} \mathbf{H}-\mathbf{N M R}\left(600 \mathrm{MHz}, \mathrm{CDCl}_{3}\right): \delta / \mathrm{ppm}=8.03-8.00(\mathrm{~m}, 2 \mathrm{H}), 7.86-7.83(\mathrm{~m}, 2 \mathrm{H}), 7.59-7.55$ (m, 1H), 7.49-7.40 (m, 4H), $4.35(\mathrm{~s}, 2 \mathrm{H}), 2.94(\mathrm{t}, J=7.4 \mathrm{~Hz}, 2 \mathrm{H}), 1.73-1.67(\mathrm{~m}, 2 \mathrm{H}), 1.43-$ $1.35(\mathrm{~m}, 2 \mathrm{H}), 0.94(\mathrm{t}, J=7.4 \mathrm{~Hz}, 3 \mathrm{H})$.

${ }^{13}$ C-NMR $\left(150 \mathrm{MHz}, \mathrm{CDCl}_{3}\right): \delta / \mathrm{ppm}=200.4,197.0,137.4,136.4,135.0,134.1,133.4$, $129.2,128.8,128.7,128.5,126.7,45.1,38.4,26.4,22.4,13.9$.

IR (Diamond-ATR, neat): $\widetilde{v} / \mathrm{cm}^{-1}=2956$ (w), 2932 (w), 1678 (vs), 1594 (m), 1580 (m), 1446 (m), 1328 (m), 1266 (m), 1206 (s), 1164 (m), 994 (m), 974 (m), 748 (s), 692 (vs).

MS (EI, $70 \mathrm{eV}): \mathrm{m} / \mathrm{z}(\%)=280\left(\mathrm{M}^{+}, 6\right), 223$ (6), 105 (100), 77 (17).

HRMS $\left(\mathbf{C}_{19} \mathbf{H}_{20} \mathbf{O}_{2}\right)$ : calc.: 280.1463; found: $280.1439\left(\mathrm{M}^{+}\right)$.

\section{1-\{3-[2-(3,4-dichlorophenyl)-2-hydroxyethyl]phenyl\}pentan-1-one (4n):}<smiles>CC(C)CC(=O)c1cccc(CC(O)c2ccc(Cl)c(Cl)c2)c1</smiles>

According to TP3 3-pentanoylbenzylzinc chloride (1g) (2.4 mL, 2.6 mmol, $1.08 \mathrm{M}$ in THF) was reacted with 3,4-dichlorobenzaldehyde (3f) (350 mg, $2.0 \mathrm{mmol}$, in $1.5 \mathrm{~mL}$ THF). After $5.5 \mathrm{~h}$ the reaction mixture was quenched with sat. aqueous $\mathrm{NH}_{4} \mathrm{Cl}$ solution. Purification by flash chromatography (silica gel, pentane $/ \mathrm{Et}_{2} \mathrm{O}=2: 1$ ) afforded the alcohol 4n (665 mg, $95 \%)$ as a white solid.

$\operatorname{mp}\left({ }^{\circ} \mathrm{C}\right)=46.8-47.8$.

${ }^{1} \mathbf{H}-\mathbf{N M R}\left(300 \mathrm{MHz}, \mathrm{CDCl}_{3}\right): \delta / \mathrm{ppm}=7.84-7.80(\mathrm{~m}, 1 \mathrm{H}), 7.75-7.72(\mathrm{~m}, 1 \mathrm{H}), 7.45-7.30$ (m, $4 \mathrm{H}), 7.16-7.11(\mathrm{~m}, 1 \mathrm{H}), 4.92-4.85(\mathrm{~m}, 1 \mathrm{H}), 3.04-3.00(\mathrm{~m}, 2 \mathrm{H}), 2.01(\mathrm{t}, J=7.4 \mathrm{~Hz}, 2 \mathrm{H})$, 2.11-1.93 (s, 1H), 1.76-1.62 (m, 2H), 1.47-1.32 (m, 2H), 0.94 (t, J=7.3 Hz, 3H).

${ }^{13}$ C-NMR $\left(75 \mathrm{MHz}, \mathrm{CDCl}_{3}\right): \delta / \mathrm{ppm}=200.5,143.8,137.8,137.4,134.1,132.6,131.5$, $130.4,129.0,128.8,127.9,126.7,125.2,73.9,45.7,38.4,26.5,22.5,13.9$.

IR (Diamond-ATR, neat): $\widetilde{v} / \mathrm{cm}^{-1}=3434$ (w), $2871(\mathrm{w}), 2930(\mathrm{~m}), 1583(\mathrm{~m}), 2956(\mathrm{~m})$, 1379 (m), 885 (m), 1179 (m), 656 (m), 730 (m), 1163 (m), 1261 (m), 787 (m), 1042 (s), 1057 (s), 1466 (s), 675 (s), 820 (s), 1673 (s), 692 (s), 1028 (vs).

MS (EI, $70 \mathrm{eV}): \mathrm{m} / \mathrm{z}(\%)=350\left(\mathrm{M}^{+},<1\right), 293$ (7), 177 (15), 176 (100), 175 (14), 119 (8). 
HRMS $\left(\mathbf{C}_{19} \mathbf{H}_{20} \mathbf{C l}_{2} \mathbf{O}_{2}\right)$ : calc.: 350.0840 ; found: $350.0839\left(\mathrm{M}^{+}\right)$.

Ethyl 2-[2-(3-propionylphenyl)ethyl]acrylate (40):<smiles>C=C(CCc1cccc(C(=O)CC)c1)C(=O)OCC</smiles>

To a solution of ethyl 2-bromomethylacrylate (31) $(560 \mathrm{mg}, 2.90 \mathrm{mmol})$ in $1.5 \mathrm{~mL}$ THF at $-60{ }^{\circ} \mathrm{C}$ was added 3-propionylbenzylzinc chloride (1h) $(2.80 \mathrm{~mL}, 3.48 \mathrm{mmol}, 1.25 \mathrm{M}$ in THF) followed by adding $\mathrm{CuCN} \cdot 2 \mathrm{LiCl}$ solution ( 2 drops). The reaction mixture was stirred at $-60{ }^{\circ} \mathrm{C}$ for $30 \mathrm{~min}$, followed by stirring at $0{ }^{\circ} \mathrm{C}$ for additional $30 \mathrm{~min}$. Then, the reaction mixture was quenched by adding sat. aqueous $\mathrm{NH}_{4} \mathrm{Cl}$ solution. The layers were separated and the aqueous layer was extracted with $\mathrm{Et}_{2} \mathrm{O}(3 \times 20 \mathrm{~mL})$. The combined extracts were dried over $\mathrm{MgSO}_{4}$. Evaporation of the solvents in vacuo and purification by flash chromatography ( silica gel, pentane / $\left.\mathrm{Et}_{2} \mathrm{O}=95: 5\right)$ afforded the acrylate $40(694 \mathrm{mg}, 92 \%$ ) as a pale yellow liquid.

${ }^{1} \mathbf{H}-\mathbf{N M R}\left(300 \mathrm{MHz}, \mathrm{CDCl}_{3}\right): \delta / \mathrm{ppm}=7.80-7.74(\mathrm{~m}, 2 \mathrm{H}), 7.39-7.34(\mathrm{~m}, 2 \mathrm{H}), 6.16-6.14$ $(\mathrm{m}, 1 \mathrm{H}), 5.48$ (q, $J=1.3 \mathrm{~Hz}, 1 \mathrm{H}), 4.21$ (q, $J=7.1 \mathrm{~Hz}, 2 \mathrm{H}), 2.98$ (q, $J=7.1 \mathrm{~Hz}, 2 \mathrm{H}), 2.89-$ $2.80(\mathrm{~m}, 2 \mathrm{H}), 2.66-2.59(\mathrm{~m}, 2 \mathrm{H}), 1.30$ (t, $J=7.2 \mathrm{~Hz}, 3 \mathrm{H}), 1.21(\mathrm{t}, J=7.3 \mathrm{~Hz}, 3 \mathrm{H})$.

${ }^{13}$ C-NMR $\left(75 \mathrm{MHz}, \mathrm{CDCl}_{3}\right): \delta / \mathrm{ppm}=200.9,167.0,141.9,139.8,137.1,133.1,128.5$, $128.0,125.8,125.4,60.7,34.8,33.8,31.8,14.2,8.3$.

IR (Diamond-ATR, neat): $\widetilde{v} / \mathrm{cm}^{-1}=2978$ (w), 2938 (w), 1712 (vs), 1684 (vs), 1300 (m), 1240 (s), 1184 (vs), 1164 (s), 1132 (s), 1028 (m), 944 (m), 782 (s), 694 (s).

MS (EI, $70 \mathrm{eV}): \mathrm{m} / \mathrm{z}(\%)=260\left(\mathrm{M}^{+}, 23\right), 232$ (16), 231 (100), 214 (11), 213 (11), 185 (16), 147 (28), 129 (14), 128 (12), 118 (10), 91 (12), 90 (19), 57 (15).

HRMS $\left(\mathbf{C}_{16} \mathbf{H}_{20} \mathbf{O}_{3}\right)$ : calc.: 260.1412; found: $260.1419\left(\mathrm{M}^{+}\right)$.

\section{1-(3-Acetylphenyl)-4,4-dimethylpentan-2-one (4p):}<smiles>CC(=O)c1cccc(CC(=O)CC(C)(C)C)c1</smiles>

According to TP4 3,3-dimethylbutyryl chloride (3e) (192 mg, $1.44 \mathrm{mmol}$ ) was added dropwise to a mixture of $\mathrm{CuCN} \cdot 2 \mathrm{LiCl}(1.88 \mathrm{~mL}, 1.88 \mathrm{mmol})$ and 3-acetylbenzylzinc chloride (1i) $\left(1.68 \mathrm{~mL}, 1.88 \mathrm{mmol}, 1.12 \mathrm{M}\right.$ in THF) at $-60{ }^{\circ} \mathrm{C}$. The reaction mixture was stirred at 
$-20{ }^{\circ} \mathrm{C}$ overnight and quenched with a mixture of sat. aqueous $\mathrm{NH}_{4} \mathrm{Cl} / \mathrm{NH}_{3}(25 \%$ in $\left.\mathrm{H}_{2} \mathrm{O}\right)=5: 1\left(25 \mathrm{~mL}\right.$ ). Purification by flash chromatography (silica gel, pentane $/ \mathrm{Et}_{2} \mathrm{O}=7: 3$ ) afforded the ketone 4 p (248 $\mathrm{mg}, 74 \%$ ) as a pale yellow liquid.

${ }^{1} \mathbf{H}-\mathbf{N M R}\left(600 \mathrm{MHz}, \mathrm{CDCl}_{3}\right): \delta / \mathrm{ppm}=7.85-7.82(\mathrm{~m}, 1 \mathrm{H}), 7.75-7.74(\mathrm{~m}, 1 \mathrm{H}), 7.43-7.40$ (m, 1H), 7.38-7.36 (m, 1H), $3.73(\mathrm{~s}, 2 \mathrm{H}), 2.58(\mathrm{~s}, 3 \mathrm{H}), 2.37$ (s, 2H), $1.00(\mathrm{~s}, 9 \mathrm{H})$.

${ }^{13}$ C-NMR $\left(150 \mathrm{MHz}, \mathrm{CDCl}_{3}\right): \delta / \mathrm{ppm}=207.1,197.9,137.4,134.7,134.2,129.2,128.8$, $127.0,54.4,51.5,31.1,29.6,26.6$.

IR (Diamond-ATR, neat): $\widetilde{v} / \mathrm{cm}^{-1}=2868$ (w), 920 (w), 1602 (w), 1584 (w), 2953 (m), 1439 (m), 790 (m), 1063 (m), 1083 (m), 1189 (m), 1713 (m), 1356 (s), 693 (s), 1269 (s), 1681 (vs). MS (EI, $70 \mathrm{eV}): \mathrm{m} / \mathrm{z}(\%)=232\left(\mathrm{M}^{+}, 3\right), 134$ (18), 133 (50), 99 (100), 90 (15), 71 (17), 57 (72), 43 (27).

HRMS $\left(\mathbf{C}_{\mathbf{1 5}} \mathbf{H}_{\mathbf{2 0}} \mathbf{O}_{2}\right)$ : calc.: 232.1463; found: $232.1447\left(\mathrm{M}^{+}\right)$.

\section{5,5-Dimethyl-2-phenylhexan-3-one (4q):}<smiles>CC(CC(=O)CC(C)(C)C)c1ccccc1</smiles>

According to TP4 3,3-dimethylbutyryl chloride (3e) (382 mg, $2.84 \mathrm{mmol}$ ) was added dropwise to a mixture of $\mathrm{CuCN} \cdot 2 \mathrm{LiCl}(3.90 \mathrm{~mL}, 3.90 \mathrm{mmol})$ and 1-phenylethylzinc chloride (1j) $\left(2.73 \mathrm{~mL}, 3.90 \mathrm{mmol}, 1.43 \mathrm{M}\right.$ in THF) at $-60{ }^{\circ} \mathrm{C}$. The reaction mixture was allowed to reach $25{ }^{\circ} \mathrm{C}$ overnight and was quenched with a mixture of sat. aqueous $\mathrm{NH}_{4} \mathrm{Cl} / \mathrm{NH}_{3}(25 \%$ in $\left.\mathrm{H}_{2} \mathrm{O}\right)=9: 1(25 \mathrm{~mL})$. Purification by flash chromatography (silica gel, pentane $/ \mathrm{Et}_{2} \mathrm{O}=95: 5$ ) afforded the ketone $\mathbf{4 q}$ (556 $\mathrm{mg}, 96 \%$ ) as a colourless liquid.

${ }^{1} \mathbf{H}-\mathbf{N M R}\left(600 \mathrm{MHz}, \mathrm{CDCl}_{3}\right): \delta / \mathrm{ppm}=7.39-7.35(\mathrm{~m}, 2 \mathrm{H}), 7.32-7.28(\mathrm{~m}, 1 \mathrm{H}), 7.26-7.23$ (m, 2H), $3.76(\mathrm{q}, J=6.9 \mathrm{~Hz}, 1 \mathrm{H}), 2.37$ (d, $J=15.3 \mathrm{~Hz}, 1 \mathrm{H}), 2.23$ (d, $J=15.5 \mathrm{~Hz}, 1 \mathrm{H}), 1.40$ (d, $J=6.9 \mathrm{~Hz}, 3 \mathrm{H}), 1.00$ (s, 9H).

${ }^{13}$ C-NMR $\left(150 \mathrm{MHz}, \mathrm{CDCl}_{3}\right): \delta / \mathrm{ppm}=210.3,140.5,128.8,128.0,127.0,54.4,53.2,30.9$, 29.6, 17.4 .

IR (Diamond-ATR, neat): $\widetilde{v} / \mathrm{cm}^{-1}=2952$ (m), 2868 (w), 1712 (s), 1492 (w), 1452 (m), 1364 (m), 1068 (w), 1044 (w), 1028 (w), 1016 (w), 912 (w), 756 (m), 700 (vs), 548 (m), $520(\mathrm{w})$. MS (EI, $70 \mathrm{eV}): \mathrm{m} / \mathrm{z}(\%)=204\left(\mathrm{M}^{+}, 3\right), 105$ (63), 99 (74), 83 (14), 79 (11), 71 (29), 69 (13), 57 (100), 55 (13), 43 (23).

HRMS ( $\left.\mathbf{C}_{\mathbf{1 4}} \mathbf{H}_{20} \mathbf{O}\right)$ : calc.: 204.1514; found: $204.1525\left(\mathrm{M}^{+}\right)$. 
Ethyl 2-[2-(3,4,5-trimethoxyphenyl)ethyl]acrylate (4r):

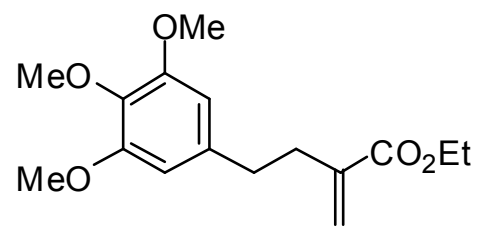

To a solution of ethyl 2-bromomethylacrylate (31) $(579 \mathrm{mg}, 3.00 \mathrm{mmol})$ in $1.5 \mathrm{~mL}$ THF at $-60{ }^{\circ} \mathrm{C}$ was added 3,4,5-trimethoxybenzylzinc chloride (1k) $(7.40 \mathrm{~mL}, 3.75 \mathrm{mmol}, 0.51 \mathrm{M}$ in $\mathrm{THF}$ ) followed by adding $\mathrm{CuCN} \cdot 2 \mathrm{LiCl}$ solution ( 3 drops). The reaction mixture was stirred at $-60{ }^{\circ} \mathrm{C}$ for $30 \mathrm{~min}$, followed by stirring at $0{ }^{\circ} \mathrm{C}$ for additional $30 \mathrm{~min}$. Then, the reaction mixture was quenched by adding sat. aqueous $\mathrm{NH}_{4} \mathrm{Cl}$ solution. The layers were separated and the aqueous layer was extracted with $\mathrm{Et}_{2} \mathrm{O}(3 \times 20 \mathrm{~mL})$. The combined extracts were dried over $\mathrm{MgSO}_{4}$. Evaporation of the solvents in vacuo and purification by flash chromatography (silica gel, pentane / $\left.\mathrm{Et}_{2} \mathrm{O}=7: 1\right)$ afforded the acrylate $\mathbf{4 0}(867 \mathrm{mg}, 98 \%)$ as colourless liquid. ${ }^{1}$ H-NMR $\left(300 \mathrm{MHz}, \mathrm{CDCl}_{3}\right): \delta / \mathrm{ppm}=6.36(\mathrm{~s}, 2 \mathrm{H}), 6.11(\mathrm{~s}, 1 \mathrm{H}), 5.48(\mathrm{~s}, 1 \mathrm{H}), 4.17(\mathrm{q}$, $J=7.1 \mathrm{~Hz}, 2 \mathrm{H}), 3.79(\mathrm{~s}, 6 \mathrm{H}), 3.77,(\mathrm{~s}, 3 \mathrm{H}), 2.73-2.63(\mathrm{~m}, 2 \mathrm{H}), 2.62-2.51(\mathrm{~m}, 2 \mathrm{H}), 1.26(\mathrm{t}$, $J=7.2 \mathrm{~Hz}, 3 \mathrm{H})$.

${ }^{13}$ C-NMR $\left(75 \mathrm{MHz}, \mathrm{CDCl}_{3}\right): \delta / \mathrm{ppm}=167.3,153.3,140.3,137.5,136.4,125.3,105.6,61.0$, $60.8,56.2,35.6,34.2,14.4$.

IR (Diamond-ATR, neat): $\widetilde{v} / \mathrm{cm}^{-1}=2936$ (w), 2840 (w), 1712 (m), 1588 (m), 1508 (m), 1456 (m), 1420 (m), 1332 (m), 1236 (s), 1184 (s), 1120 (vs), 1008 (m), 944 (m), 820 (m).

MS (EI, $70 \mathrm{eV}): \mathrm{m} / \mathrm{z}(\%)=294$ (31), 182 (20), 181 (100), 148 (7), 121 (9).

HRMS ( $\left.\mathbf{C}_{\mathbf{1 6}} \mathbf{H}_{\mathbf{2 2}} \mathbf{O}_{\mathbf{5}}\right)$ : calc.: 294.1467; found: $294.1457\left(\mathrm{M}^{+}\right)$.

\section{1-(6-Chloro-1,3-benzodioxol-5-yl)-4,4-dimethylpentan-2-one (4s):}<smiles>CC(C)(C)CC(=O)Cc1cc2c(cc1Cl)OCO2</smiles>

According to TP4 2,3-dimethylpropionyl chloride (3j) (377 mg, $2.80 \mathrm{mmol}$ ) was added dropwise to a mixture of $\mathrm{CuCN} \cdot 2 \mathrm{LiCl}(3.92 \mathrm{~mL}, 3.92 \mathrm{mmol})$ and 6-chloro-1,3-benzodioxol-5ylmethylzinc chloride (11) $\left(2.80 \mathrm{~mL}, 3.92 \mathrm{mmol}, 1.40 \mathrm{M}\right.$ in THF) at $-60{ }^{\circ} \mathrm{C}$. The reaction mixture was allowed to reach $25^{\circ} \mathrm{C}$ overnight and was quenched with a mixture of sat. aqueous $\mathrm{NH}_{4} \mathrm{Cl} / \mathrm{NH}_{3}\left(25 \%\right.$ in $\left.\mathrm{H}_{2} \mathrm{O}\right)=5: 1(25 \mathrm{~mL})$. Purification by flash chromatography 
(silica gel, pentane $\left./ \mathrm{Et}_{2} \mathrm{O}=95: 5\right)$ afforded the ketone $4 \mathbf{s}(703 \mathrm{mg}, 93 \%$ ) as a pale yellow liquid.

${ }^{1}$ H-NMR $\left(600 \mathrm{MHz}, \mathrm{CDCl}_{3}\right): \delta / \mathrm{ppm}=6.84(\mathrm{~s}, 1 \mathrm{H}), 6.63(\mathrm{~s}, 1 \mathrm{H}), 5.95(\mathrm{~s}, 2 \mathrm{H}), 3.70(\mathrm{~s}, 2 \mathrm{H})$, $2.38(\mathrm{~s}, 2 \mathrm{H}), 1.02(\mathrm{~s}, 9 \mathrm{H})$.

${ }^{13}$ C-NMR $\left(150 \mathrm{MHz}, \mathrm{CDCl}_{3}\right): \delta / \mathrm{ppm}=206.5,147.4,146.7,126.0,125.7,110.9,109.8$, 101.7, 54.3, 49.2, 31.0, 29.6.

IR (Diamond-ATR, neat): $\widetilde{v} / \mathrm{cm}^{-1}=2952$ (m), 2904 (w), 1716 (m), 1504 (s), 1480 (vs), 1364 (m), 1248 (s), 1232 (s), 1120 (s), 1036 (vs), 984 (m), 932 (s), 840 (s), 724 (w), 684 (w). MS (EI, $70 \mathrm{eV}):$ m/z (\%) = 268 (77), 171 (76), 169 (50), 110 (23), 99 (100), 71 (65), 57 (43), $41(33)$.

HRMS ( $\left.\mathbf{C}_{14} \mathrm{H}_{17} \mathrm{ClO}_{3}\right)$ : calc.: 268.0866; found: $268.0855\left(\mathrm{M}^{+}\right)$.

\section{Ethyl (2-chlorophenyl)acetate (5):}<smiles>CCOC(=O)Cc1ccccc1Cl</smiles>

Reaction 1 using ethyl chloroformate:

To 2-chlorobenzylzinc chloride (1a) $(2.62 \mathrm{~mL}, 4.00 \mathrm{mmol}, 1.5 \mathrm{M}$ in $\mathrm{THF})$ at $-30{ }^{\circ} \mathrm{C}$ was added THF $(0.5 \mathrm{~mL})$ followed by $\mathrm{Pd}\left(\mathrm{PPh}_{3}\right)_{4}(116 \mathrm{mg}, 5 \mathrm{~mol} \%)$. The reaction mixture was stirred for $5 \mathrm{~min}$. Then, ethyl chloroformate $(227 \mathrm{mg}, 2.09 \mathrm{mmol})$ was added dropwise. Stirring was continued for $10 \mathrm{~min}$ at $-30{ }^{\circ} \mathrm{C}$ followed by $6.25 \mathrm{~h}$ at $25^{\circ} \mathrm{C}$. The reaction mixture was quenched by adding mixture of sat. aqueous $\mathrm{NH}_{4} \mathrm{Cl} / \mathrm{NH}_{3}\left(25 \%\right.$ in $\left.\mathrm{H}_{2} \mathrm{O}\right)=4: 1(15 \mathrm{~mL})$. The layers were separated and the aqueous layer was extracted with DCM $(3 \times 50 \mathrm{~mL})$. The combined extracts were dried over $\mathrm{MgSO}_{4}$. Evaporation of the solvents in vacuo and purification by flash chromatography (silica gel, pentane $/ \mathrm{Et}_{2} \mathrm{O}=98: 2$ ) afforded the phenylacetic acid ester 5 (336 $\mathrm{mg}, 81 \%)$ as colourless liquid.

\section{Reaction 2 using ethyl cyanoformate:}

To 2-chlorobenzyl zinc chloride (1a) $(0.67 \mathrm{~mL}, 1.00 \mathrm{mmol}, 1.5 \mathrm{M}$ in $\mathrm{THF})$ at $-30{ }^{\circ} \mathrm{C}$ was added dropwise $\mathrm{TMSCH}_{2} \mathrm{Li}(1.00 \mathrm{~mL}, 1.00 \mathrm{mmol}, 1 \mathrm{M}$ in pentane). The reaction mixture was stirred for $30 \mathrm{~min}$. CuCN.2 $\mathrm{LiCl}$ solution $(1.00 \mathrm{~mL}, 1.00 \mathrm{mmol}, 1 \mathrm{M}$ in THF) was added dropwise and the mixture was stirred for additional $30 \mathrm{~min}$. Ethyl cyanoformate $(150 \mathrm{mg}$, $1.5 \mathrm{mmol}$ ) was added dropwise. Stirring was continued for $10 \mathrm{~min}$ at $-30{ }^{\circ} \mathrm{C}$ followed by $6 \mathrm{~h}$ at $0{ }^{\circ} \mathrm{C}$. The reaction mixture was quenched by adding mixture of sat. aqueous $\mathrm{NH}_{4} \mathrm{Cl} / \mathrm{NH}_{3}\left(25 \%\right.$ in $\left.\mathrm{H}_{2} \mathrm{O}\right)=2: 1(15 \mathrm{~mL})$. The layers were separated and the aqueous layer 
was extracted with DCM $(3 \times 50 \mathrm{~mL})$. The combined extracts were dried over $\mathrm{MgSO}_{4}$. Evaporation of the solvents in vacuo and purification by flash chromatography (silica gel, pentane $\left./ \mathrm{Et}_{2} \mathrm{O}=98: 2\right)$ afforded the phenylacetic acid ester $5(152 \mathrm{mg}, 77 \%)$ as colourless liquid.

${ }^{1} \mathbf{H}-\mathbf{N M R}\left(300 \mathrm{MHz}, \mathrm{CDCl}_{3}\right): \delta / \mathrm{ppm}=7.42-7.34(\mathrm{~m}, 1 \mathrm{H}), 7.32-7.17$ (m, 3H), 4.17 (q, $J=7.1 \mathrm{~Hz}, 2 \mathrm{H}), 3.76(\mathrm{~s}, 2 \mathrm{H}), 1.25(\mathrm{t}, J=7.2 \mathrm{~Hz}, 3 \mathrm{H})$.

${ }^{13}$ C-NMR $\left(150 \mathrm{MHz}, \mathrm{CDCl}_{3}\right): \delta / \mathrm{ppm}=170.5,134.5,132.5,131.4,129.4,128.6,126.8$, $61.0,39.2,14.1$.

IR (Diamond-ATR, neat): $\widetilde{v} / \mathrm{cm}^{-1}=2981(\mathrm{w}), 1367$ (m), 1335 (m), $1122(\mathrm{~m}), 1475(\mathrm{~m})$, 1445 (m), 1246 (m), 1053 (s), 681 (s), 1216 (s), 1028 (s), 741 (vs), 1156 (vs), 1731 (vs).

MS (EI, $70 \mathrm{eV}): \mathrm{m} / \mathrm{z}(\%)=198\left(\mathrm{M}^{+}, 4\right), 163$ (100), 135 (23) 127 (78), 125 (35, 89 (21).

HRMS ( $\left.\mathbf{C}_{10} \mathbf{H}_{11} \mathbf{C l O}_{2}\right)$ : calc.: 198.0448; found: $198.0462\left(\mathrm{M}^{+}\right)$.

\section{Preparation of papaverine.}

\section{Starting materials. ${ }^{4,5}$}

$N$-[(1E)-(3,4-Dimethoxyphenyl)methylene]-2,2-dimethoxyethanamine (9):<smiles>COc1ccc(/C=N/CC(OC)OC)cc1OC</smiles>

To a solution of 3,4-dimethoxybenzyaldehyde $(8.31 \mathrm{~g}, 50.0 \mathrm{mmol})$ in $150 \mathrm{~mL}$ toluene was added aminoacetaldehyde dimethylacetal $(8.24 \mathrm{~mL}, 76 \mathrm{mmol})$. The reaction mixture was refluxed for $6 \mathrm{~h}$ and the water was removed by using Dean-Stark apparatus. After cooling to $25^{\circ} \mathrm{C}$, the solvent was removed in vacuo. The yellow oil was dissolved in DCM (50 mL) and washed with water $(4 \times 50 \mathrm{~mL})$, then dried over $\mathrm{Na}_{2} \mathrm{SO}_{4}$. Evaporation of the solvents in vacuo gives the crude product $9(12.8 \mathrm{~g}, 100 \%)$ as a pale yellow solid, which was used without further purification.

$\mathbf{m p}\left({ }^{\circ} \mathrm{C}\right)=54.7-56.3$.

${ }^{1}$ H-NMR $\left(300 \mathrm{MHz}, \mathrm{CDCl}_{3}\right): \delta / \mathrm{ppm}=8.18-8.17(\mathrm{~m}, 1 \mathrm{H}), 7.41(\mathrm{~d}, J=1.9 \mathrm{~Hz}, 1 \mathrm{H}), 7.14$ $(\mathrm{dd}, J=8.1 \mathrm{~Hz}, J=1.9 \mathrm{~Hz}, 1 \mathrm{H}), 6.85(\mathrm{~d}, J=8.4 \mathrm{~Hz}, 1 \mathrm{H}), 4.65(\mathrm{t}, J=5.4 \mathrm{~Hz}, 1 \mathrm{H}), 3.91(\mathrm{~s}$, $3 \mathrm{H}), 3.89$ (s, 3H), $3.73(\mathrm{dd}, J=5.4 \mathrm{~Hz}, J=1.3 \mathrm{~Hz}, 2 \mathrm{H}), 3.40(\mathrm{~s}, 6 \mathrm{H})$.

${ }^{13}$ C-NMR $\left(150 \mathrm{MHz}, \mathrm{CDCl}_{3}\right): \delta / \mathrm{ppm}=163.0,151.4,149.2,129.3,123.3,110.3,108.8$, $103.9,63.4,55.9,55.9,54.1$.

IR (Diamond-ATR, neat): $\widetilde{v} / \mathrm{cm}^{-1}=2832$ (w), 1600 (m), 1583 (s), 1641 (s), 1422 (s), 1512 (s), 1158 (s), 868 (s), 752 (s), 809 (s), 1137 (vs), 1238 (vs), 959 (vs), 1015 (vs), 1263 (vs). 
(3,4-Dimethoxybenzyl)(2,2-dimethoxyethyl)amine (10):<smiles>COc1ccc(CNCC(OC)OC)cc1OC</smiles>

To a solution of $N$-[(1E)-(3,4-dimethoxyphenyl)methylene]-2,2-dimethoxyethanamine (9) $(12.8 \mathrm{~g}, 50.0 \mathrm{mmol})$ in ethanol $(50 \mathrm{~mL})$ was added sodium borohydride $(3.78 \mathrm{~g}, 100 \mathrm{mmol})$ and the reaction mixture was stirred for $60 \mathrm{~h}$ at $25^{\circ} \mathrm{C}$. Then, water $(150 \mathrm{~mL})$ was added carefully. The layers were separated and the aqueous phase was extracted with DCM $(3 \times 300 \mathrm{~mL})$. The combined extracts were washed with water $(3 \times 300 \mathrm{~mL})$, brine $(1 \times 300 \mathrm{~mL})$ and then dried over $\mathrm{Na}_{2} \mathrm{SO}_{4}$. . Evaporation of the solvents in vacuo gives the crude product $10(11.0 \mathrm{~g}, 86 \%)$ as a pale yellow liquid which was used without further purification.

${ }^{1}$ H-NMR $\left(300 \mathrm{MHz}, \mathrm{CDCl}_{3}\right): \delta / \mathrm{ppm}=6.88-6.85(\mathrm{~m}, 1 \mathrm{H}), 6.84-6.76(\mathrm{~m}, 2 \mathrm{H}), 4.46(\mathrm{t}$, $J=5.5 \mathrm{~Hz}, 1 \mathrm{H}), 3.86(\mathrm{~s}, 3 \mathrm{H}), 3.83(\mathrm{~s}, 3 \mathrm{H}), 3.72(\mathrm{~s}, 2 \mathrm{H}), 3.34(\mathrm{~s}, 6 \mathrm{H}), 2.72(\mathrm{~d}, J=5.8 \mathrm{~Hz}, 2 \mathrm{H})$.

${ }^{13}$ C-NMR $\left(75 \mathrm{~Hz}, \mathrm{CDCl}_{3}\right): \delta / \mathrm{ppm}=148.8,147.9,132.6,120.1,111.2,110.9,103.7,55.7$, $55.7,53.7,53.5,50.3$.

MS (EI, $\left.70 \mathrm{eV}): \mathrm{m} / \mathrm{z}(\%)=255, \mathrm{M}^{+}, 2\right), 180$ (5), 151 (100), 107 (3), 75 (14).

HRMS $\left(\mathbf{C}_{13} \mathbf{H}_{21} \mathbf{N O}_{4}\right)$ : calc.: 255.1464; found: 255.1471( $\left.{ }^{+}\right)$.

\section{$N$-(3,4-dimethoxybenzyl)- $N$-(2,2-dimethoxyethyl)-4-methylbenzenesulfonamide (11):}

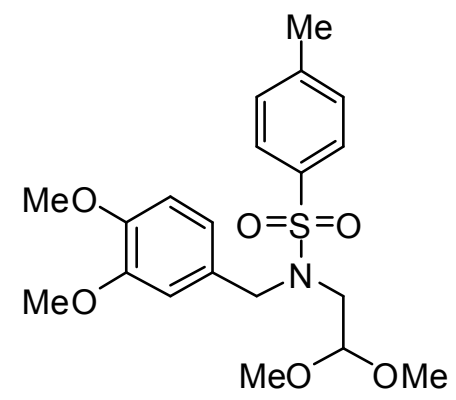

Pyridine $(3.40 \mathrm{~mL}, 42.0 \mathrm{mmol})$ was added dropwise at $0{ }^{\circ} \mathrm{C}$ to a solution of 3,4dimethoxybenzyl)(2,2-dimethoxyethyl)amine (10) $(7.66 \mathrm{~g}, 30.0 \mathrm{mmol})$ in DCM (60 mL). Tosyl chloride $(7.43 \mathrm{~g}, 39.0 \mathrm{mmol})$ was added and the reaction mixture was allowed to warm to $25{ }^{\circ} \mathrm{C}$ overnight and then poured on sat. aqueous $\mathrm{NaHCO}_{3}$ solution. The layers were separated and the aqueous phase was extracted with DCM $(3 \times 100 \mathrm{~mL})$, then dried over $\mathrm{MgSO}_{4}$. Evaporation of the solvents in vacuo and purification by flash chromatography (silica gel, pentane $\left./ \mathrm{Et}_{2} \mathrm{O}=1: 2\right)$ afforded the sulphonamide 11 (12.2 g, 99\%) as a pale yellow liquid. 
${ }^{1} \mathbf{H}-\mathbf{N M R}\left(300 \mathrm{MHz}, \mathrm{CDCl}_{3}\right): \delta / \mathrm{ppm}=7.74-7.68(\mathrm{~m}, 2 \mathrm{H}), 7.30-7.24(\mathrm{~m}, 2 \mathrm{H}), 6.73-6.71$ $(\mathrm{m}, 2 \mathrm{H}), 6.66-6.64(\mathrm{~m}, 1 \mathrm{H}), 4.38(\mathrm{~s}, 2 \mathrm{H}), 4.33(\mathrm{t}, J=5.4 \mathrm{~Hz}, 1 \mathrm{H}), 3.81(\mathrm{~s}, 3 \mathrm{H}), 3.71(\mathrm{~s}, 3 \mathrm{H})$, $3.23(\mathrm{~s}, 6 \mathrm{H}), 3.18(\mathrm{~d}, J=5.4 \mathrm{~Hz}, 2 \mathrm{H}), 2.39(\mathrm{~s}, 3 \mathrm{H})$.

${ }^{13}$ C-NMR $\left(150 \mathrm{MHz}, \mathrm{CDCl}_{3}\right): \delta / \mathrm{ppm}=149.0,148.5,143.1,137.7,129.5,128.4,127.1$, 121.0, 111.3, 110.7, 110.7, 103.8, 55.8, 55.6, 54.5, 52.2, 48.3, 21.4.

IR (Diamond-ATR, neat): $\widetilde{v} / \mathrm{cm}^{-1}=2935$ (w), 2834 (w), 1595 (w), 1514 (s), 1438 (m), 1337 (s), 1255 (s), 1236 (s), 1155 (vs), 1066 (s), 1026 (vs), 997 (s), 911 (s), 813 (s), 760 (s), 706 (m), 658 (vs).

MS (EI, $70 \mathrm{eV}): \mathrm{m} / \mathrm{z}(\%)=409\left(\mathrm{M}^{+},<1\right), 254$ (5), 151 (28), 91 (4), 75 (100).

HRMS ( $\left.\mathbf{C}_{20} \mathbf{H}_{27} \mathbf{N O}_{6} \mathrm{~S}\right)$ : calc.: 409.1559; found: $409.1546\left(\mathrm{M}^{+}\right)$.

\section{6,7-Dimethoxyisoquinoline (7):}<smiles>COc1cc2ccncc2cc1OC</smiles>

To a solution of $N$-(3,4-dimethoxybenzyl)- $N$-(2,2-dimethoxyethyl)-4methylbenzenesulfonamide (11) $(12.1 \mathrm{~g}, 29.5 \mathrm{mmol})$ in dioxane $(280 \mathrm{~mL})$ was added $6 \mathrm{~N} \mathrm{HCl}$ (22 mL). The reaction mixture was refluxed for $22 \mathrm{~h}$. After cooling to $25^{\circ} \mathrm{C}$, the solution was poured on water. The layers were separated and the aqueous phase was extracted with $\mathrm{Et}_{2} \mathrm{O}$ $(2 \times 250 \mathrm{~mL}), \mathrm{DCM}(3 \times 250 \mathrm{~mL})$. The combined aqueous phases were treated with $\mathrm{NaOH}$ $(10 \%)$ solution until $\mathrm{pH}>9$. The aqueous phase was extracted with $\mathrm{Et}_{2} \mathrm{O}(2 \times 250 \mathrm{~mL}), \mathrm{DCM}$ $(3 \times 250 \mathrm{~mL})$ and the combined extracts were dried over $\mathrm{MgSO}_{4}$. Evaporation of the solvents in vacuo and purification by flash chromatography (silica gel, EtOAc) afforded the isoquinoline $7(4.78 \mathrm{~g}, 86 \%)$ as a white solid.

$\mathbf{m p}\left({ }^{\circ} \mathrm{C}\right)=93.3-95.4$.

${ }^{1}$ H-NMR $\left(300 \mathrm{MHz}, \mathrm{CDCl}_{3}\right): \delta / \mathrm{ppm}=8.98(\mathrm{~s}, 1 \mathrm{H}), 8.33(\mathrm{~d}, J=5.6 \mathrm{~Hz}, 1 \mathrm{H}), 7.42(\mathrm{~d}$, $J=5.83 \mathrm{~Hz} .1 \mathrm{H}), 7.11(\mathrm{~s}, 1 \mathrm{H}), 6.98(\mathrm{~s}, 1 \mathrm{H}), 3.95$ (s, 3H).

${ }^{13}$ C-NMR $\left(150 \mathrm{MHz}, \mathrm{CDCl}_{3}\right): \delta / \mathrm{ppm}=152.8,150.1,149.8,141.8,132.3,124.6,119.0$, 105.1, 104.4, 55.9, 55.9 .

IR (Diamond-ATR, neat): $\widetilde{v} / \mathrm{cm}^{-1}=3015$ (w), 2836 (w), 1573 (m), 1502 (s), 1477 (s), 1459 (m), 1433 (m), 1412 (s), 1335 (s), 1248 (vs), 1206 (s), 1138 (vs), 1001 (s), 923 (s), 852 (vs), $755(\mathrm{~s}), 632(\mathrm{~s})$.

MS (EI, $70 \mathrm{eV}): \mathrm{m} / \mathrm{z}(\%)=189\left(\mathrm{M}^{+}, 100\right), 174$ (11), 146 (24), 117 (8), 103 (6), 91 (6).

HRMS ( $\left.\mathbf{C}_{\mathbf{1 1}} \mathbf{H}_{\mathbf{1 1}} \mathbf{N O}_{2}\right)$ : calc.: 189.0790; found: $189.0788\left(\mathrm{M}^{+}\right)$. 


\section{1-Iodo-6,7-dimethoxyisoquinoline (8):}<smiles>COc1cc2ccnc(I)c2cc1OC</smiles>

To a solution of 6,7-dimethoxyisoquinoline (7) $(946 \mathrm{mg}, 5.00 \mathrm{mmol})$ in THF (5 mL) was added TMPMgCl$\cdot \mathrm{LiCl}(5.13 \mathrm{~mL}, 6.00 \mathrm{mmol}, 1.17 \mathrm{M}$ in THF $)$ at $25^{\circ} \mathrm{C}$. The reaction mixture was stirred for $4 \mathrm{~h}$. Iodine $(1.52 \mathrm{~g}, 6.00 \mathrm{mmol})$ was dissolved in THF $(3 \mathrm{~mL})$ in a second flask at $-40{ }^{\circ} \mathrm{C}$. To this solution, the magnesium compound was added dropwise. The solution was stirred $10 \mathrm{~min}$ at $-40{ }^{\circ} \mathrm{C}$, then $10 \mathrm{~min}$ at $0{ }^{\circ} \mathrm{C}$. The reaction mixture was quenched by adding a mixture of sat. aqueous $\mathrm{NH}_{4} \mathrm{Cl}$ solution and sat. aqueous $\mathrm{Na}_{2} \mathrm{~S}_{2} \mathrm{O}_{3}$ solution, then sat. aqueous $\mathrm{NaHCO}_{3}$ until $\mathrm{pH}>7$. The layers were separated and the aqueous phase was extracted with DCM $(3 \times 100 \mathrm{~mL})$, then dried over $\mathrm{MgSO}_{4}$. Evaporation of the solvents in vacuo and purification by flash chromatography (silica gel, pentane $/ \mathrm{Et}_{2} \mathrm{O}=1: 4$ ) afforded the isoquinoline $8(1.14 \mathrm{~g}, 73 \%)$ as a pale yellow solid.

$\mathbf{m p}\left({ }^{\circ} \mathrm{C}\right)=140.0-141.1$ (decomposition).

${ }^{1}$ H-NMR $\left(300 \mathrm{MHz}, \mathrm{CDCl}_{3}\right): \delta / \mathrm{ppm}=8.06(\mathrm{~d}, J=5.6 \mathrm{~Hz}, 1 \mathrm{H}), 7.37(\mathrm{~d}, J=5.1 \mathrm{~Hz}, 1 \mathrm{H})$, $7.29(\mathrm{~s}, 1 \mathrm{H}), 6.59(\mathrm{~s}, 1 \mathrm{H}), 4.03(\mathrm{~s}, 3 \mathrm{H}), 4.00(\mathrm{~s}, 3 \mathrm{H})$.

${ }^{13}$ C-NMR $\left(150 \mathrm{MHz}, \mathrm{CDCl}_{3}\right): \delta / \mathrm{ppm}=153.3,151.3,141.8,132.4,127.9,124.6,120.0$, $111.0,105.0,56.3,56.1$.

IR (Diamond-ATR, neat): $\tilde{v} / \mathrm{cm}^{-1}=2936$ (w), 1504 (s), 1473 (s), 1458 (s), 1431 (s), 1392 (s), 1296 (s), 1251 (s), 1226 (s), 1140 (vs), 1006 (s), 929 (s), 858 (vs), 774 (s), 671 (s).

MS (EI, $70 \mathrm{eV}): \mathrm{m} / \mathrm{z}(\%)=314\left(\mathrm{M}^{+}, 539\right), 189$ (12), 188 (100), 145 (3), 94 (6).

HRMS ( $\left.\mathbf{C}_{11} \mathbf{H}_{10} \mathrm{INO}_{2}\right)$ : calc.: 314.9751 ; found: $314.9756\left(\mathrm{M}^{+}\right)$.

\section{4-(Chloromethyl)-1,2-dimethoxybenzene (2m):}

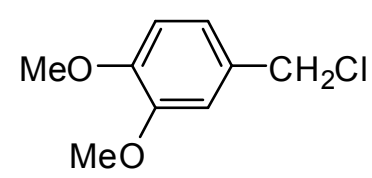

To a solution of $\mathrm{LiCl}\left(6.87 \mathrm{~g}, 60.0 \mathrm{mmol}\right.$, dried for $10 \mathrm{~min}$ under high vacuum at $400{ }^{\circ} \mathrm{C}$ using a heat gun) in THF $(50 \mathrm{~mL})$ was added 3,4-dimethoxybenzylic alcohol (3.30 g, $20.0 \mathrm{mmol})$ at $0{ }^{\circ} \mathrm{C}$. Then, $\mathrm{NEt}_{3}(5.60 \mathrm{~mL}, 40.0 \mathrm{mmol})$ was added dropwise, followed by mesyl chloride $(2.33 \mathrm{~mL}, 30.0 \mathrm{mmol})$. The reaction mixture was allowed to reach $25^{\circ} \mathrm{C}$ overnight. Then, DCM $(300 \mathrm{~mL})$ was added and the solution was washed with water ( $3 \times 250 \mathrm{~mL}$ ). The combined extracts were dried over $\mathrm{MgSO}_{4}$. Evaporation of the solvents in 
vacuo and purification by flash chromatography (silica gel, pentane $/ \mathrm{Et}_{2} \mathrm{O}=4: 1$ ) afforded the benzylic chloride $\mathbf{2 m}(2.56 \mathrm{~g}, 69 \%)$ as a white solid.

$\mathbf{m p}\left({ }^{\circ} \mathrm{C}\right)=54.8-56.0$.

${ }^{1} \mathbf{H}-\mathrm{NMR}\left(300 \mathrm{MHz}, \mathrm{CDCl}_{3}\right): \delta / \mathrm{ppm}=6.94-6.88(\mathrm{~m}, 1 \mathrm{H}), 6.89(\mathrm{~s}, 1 \mathrm{H}), 6.83-6.77(\mathrm{~m}, 1 \mathrm{H})$, $4.54(\mathrm{~s}, 2 \mathrm{H}), 3.87(\mathrm{~s}, 3 \mathrm{H}), 3.85(\mathrm{~s}, 3 \mathrm{H})$.

${ }^{13}$ C-NMR $\left(150 \mathrm{MHz}, \mathrm{CDCl}_{3}\right): \delta / \mathrm{ppm}=149.1,149.0,129.9,121.0,111.6,110.9,55.8,55.8$, 46.6.

IR (Diamond-ATR, neat): $\tilde{v} / \mathrm{cm}^{-1}=3010$ (w), 2935 (w), 2838 (w), 1593 (m), 1514 (s), 1463

(s), 1450 (m), 1437 (m), 1259 (s), 1232 (vs), 1154 (vs), 1139 (vs), 1036 (s), 1022 (vs), 848 (s), $815(\mathrm{~s}), 685$ (vs).

MS (EI, $70 \mathrm{eV}): \mathrm{m} / \mathrm{z}(\%)=186\left(\mathrm{M}^{+}, 17\right), 151$ (100), 107 (9), 91 (3), 77 (4).

HRMS ( $\left.\mathbf{C}_{9} \mathbf{H}_{11} \mathbf{C l O}_{2}\right)$ : calc.: 186.0448; found: $186.0434\left(\mathrm{M}^{+}\right)$.

\section{3,4,-Dimethoxybenzyl zinc chloride (1m):}

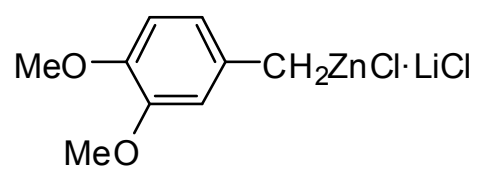

According to TP2 3,4-dimethoxybenzyl chloride (2m) (933 mg, $5.00 \mathrm{mmol}$, in $2 \mathrm{~mL}$ THF) was added dropwise at $0{ }^{\circ} \mathrm{C}$ to a suspension of $\mathrm{LiCl}(848 \mathrm{mg}, 20.0 \mathrm{mmol})$ and zinc dust $(1.31 \mathrm{~g}, 20.0 \mathrm{mmol})$ in $2 \mathrm{~mL}$ THF (activation: $\mathrm{BrCH}_{2} \mathrm{CH}_{2} \mathrm{Br}(0.02 \mathrm{~mL}, 5 \mathrm{~mol} \%)$, TMSCl ( 3 drops, $1 \mathrm{~mol} \%)$ ). The reaction mixture was stirred for $2 \mathrm{~h}$ at $0{ }^{\circ} \mathrm{C}$ followed by stirring for $2.5 \mathrm{~h}$ at $25{ }^{\circ} \mathrm{C}$. After centrifugation iodometric titration ${ }^{2}$ of $1 \mathrm{~m}$ indicates a yield of $72 \%$.

\section{Papaverine (6):}<smiles>COc1ccc(Cc2nccc3cc(OC)c(OC)cc23)cc1OC</smiles>

To a solution of 1-iodo-6,7-dimethoxyisoquinoline (8) $(315 \mathrm{mg}, 1.00 \mathrm{mmol})$ in THF (3 mL) was added S-Phos (20.5 mg, $0.05 \mathrm{mmol}, 5 \mathrm{~mol} \%), \mathrm{Pd}(\mathrm{OAc})_{2} \quad(5.61 \mathrm{mg}, 0.03 \mathrm{mmol}$, $2.5 \mathrm{~mol} \%)$. Then, 3,4,-dimethoxybenzylzinc chloride (1 m) $(2.0 \mathrm{~mL}, 1.40 \mathrm{mmol}, 0.70 \mathrm{M}$ in THF) was added dropwise. The reaction mixture was stirred for $1.25 \mathrm{~h}$ at $25^{\circ} \mathrm{C}$, then 
quenched by adding a mixture of sat. aqueous $\mathrm{NH}_{4} \mathrm{Cl} / \mathrm{NH}_{3}\left(25 \%\right.$ in $\left.\mathrm{H}_{2} \mathrm{O}\right)=5: 1$. The layers were separated and the aqueous layer was extracted with DCM $(5 \times 50 \mathrm{~mL})$. The combined extracts were dried over $\mathrm{MgSO}_{4}$. Evaporation of the solvents in vacuo and purification by flash chromatography (silica gel, pentane $/ \mathrm{Et}_{2} \mathrm{O}=1: 4,2 \% \mathrm{NEt}_{3}, 2 \% \mathrm{EtOH}$ ) afforded papaverine 6 (229 $\mathrm{mg}, 68 \%$ ) as pale yellow solid.

$\mathbf{m p}\left({ }^{\circ} \mathrm{C}\right)=144.2-145.8$.

${ }^{1}$ H-NMR $\left(300 \mathrm{MHz}, \mathrm{CDCl}_{3}\right): \delta / \mathrm{ppm}=8.35(\mathrm{~d}, J=6.0 \mathrm{~Hz}, 1 \mathrm{H}), 7.46(\mathrm{~d}, J=5.7 \mathrm{~Hz}, 1 \mathrm{H})$, $7.37(\mathrm{~s}, 1 \mathrm{H}), 7.06(\mathrm{~s}, 1 \mathrm{H}), 6.83-6.80(\mathrm{~m}, 2 \mathrm{H}), 6.75(\mathrm{~d}, J=8.1 \mathrm{~Hz}, 1 \mathrm{H}), 4.58(\mathrm{~s}, 2 \mathrm{H}), 4.00(\mathrm{~s}$, $3 \mathrm{H}), 3.91(\mathrm{~s}, 3 \mathrm{H}), 3.81(\mathrm{~s}, 3 \mathrm{H}), 3.77(\mathrm{~s}, 3 \mathrm{H})$.

${ }^{13}$ C-NMR $\left(150 \mathrm{MHz}, \mathrm{CDCl}_{3}\right): \delta / \mathrm{ppm}=157.7,152.3,149.7,149.0,147.4,140.9,133.4$, 132.2, 122.8, 120.4, 118.6, 111.8, 111.1, 105.2, 104.1, 55.9, 55.8, 55.8, 55.7, 42.2.

IR (Diamond-ATR, neat): $\widetilde{v} / \mathrm{cm}^{-1}=2956$ (w),2939 (w),2835 (w),1504 (vs),1478 (s),1463 (s),1454 (m),1434 (s),1414 (s),1257 (vs),1232 (vs),1202 (s),1157 (s),1153 (s),1147 (s),1139 (vs),1075 (m),1045 (m),1028 (vs),986 (s),875 (s),867 (m),860 (s),843 (s),822 (s),805 (m),785 (s),768 (m),736 (m),732 (m),661 (s),645 (m).

MS (EI, $70 \mathrm{eV}): \mathrm{m} / \mathrm{z}(\%)=339\left(\mathrm{M}^{+}, 55\right), 324$ (75), 308 (20), 154 (13).

HRMS ( $\left.\mathbf{C}_{\mathbf{2 0}} \mathbf{H}_{21} \mathbf{N O}_{4}\right)$ : calc.: 339.1471; found: $339.1455\left(\mathrm{M}^{+}\right)$.

\section{$\underline{\text { References: }}$}

1) Knochel, P.; Yeh, M. C. P.; Berk, S. C.; Talbert, J. J. Org. Chem. 1988, 53, 2390.

2) Krasovskiy, A.; Knochel, P. Synthesis 2006, 5, 890.

3) Zinc dust, commercial available by Aldrich; $<10$ micron.

4) Product 9 and $\mathbf{1 0}$ were prepared according to (a) Arnold, N. J.; Arnold, R.; Beer, D.; Bhalay, G.; Collingwood, S. P.; Craig, S.; Devereux, N.; Dodds, M.; Dunstan, A. R.; Fairhurst, R. A.; Farr, D.; Fullerton, J. D.; Glen, A.; Gomez, S.; Haberthuer, S.; Hatto, J. D. I.; Howes, C.; Jones, D.; Keller, T. H.; Leuenberger, B.; Moser, H. E.; Muller, I.; Naef, R.; Nicklin, P. A.; Sandham, D. A.; Turner, K. L.; Tweed, M. F.; Watson, S. J.; Zurini, M. Bioorg. \& Medicinal Chem. Lett. 2007, 17, 2376; (b) Ioanaviciu, A.; Antony, S.; Pommier, Y.; Staker, B. L.; Stewart, L.; Cushman, M. J. Med. Chem. 2005, 48, 4803.

5) Product 7 was prepared according to: Boger, D. L.; Brotherton, C. E.; Kelley, M. D. Tetrahedron 1981, 37, 5181. 


\section{NMR-SPECTRA}

3-(Chloromethyl)benzonitrile (2f)
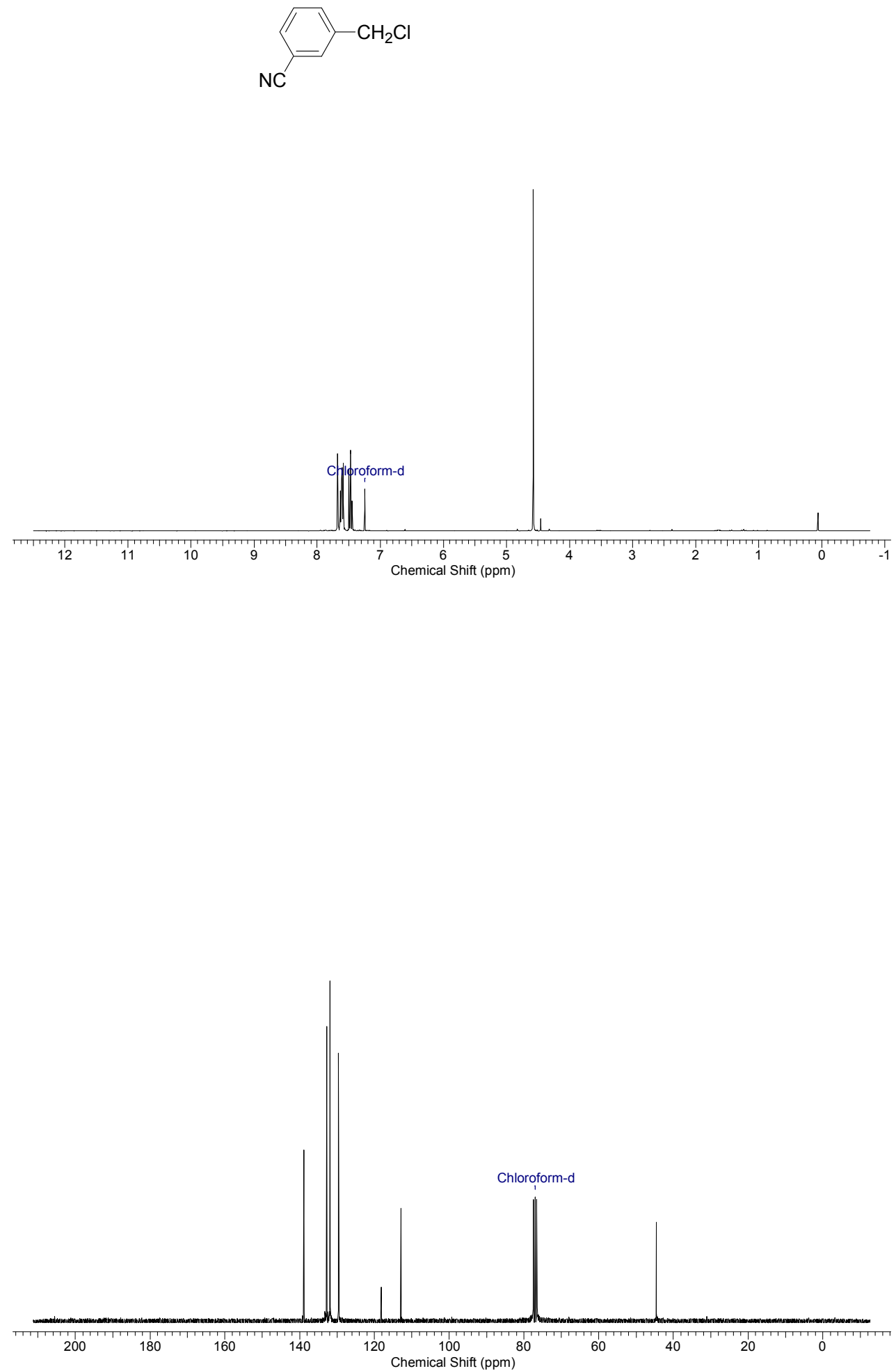
1-[3-(Chloromethyl)phenyl]pentan-1-one (2g)
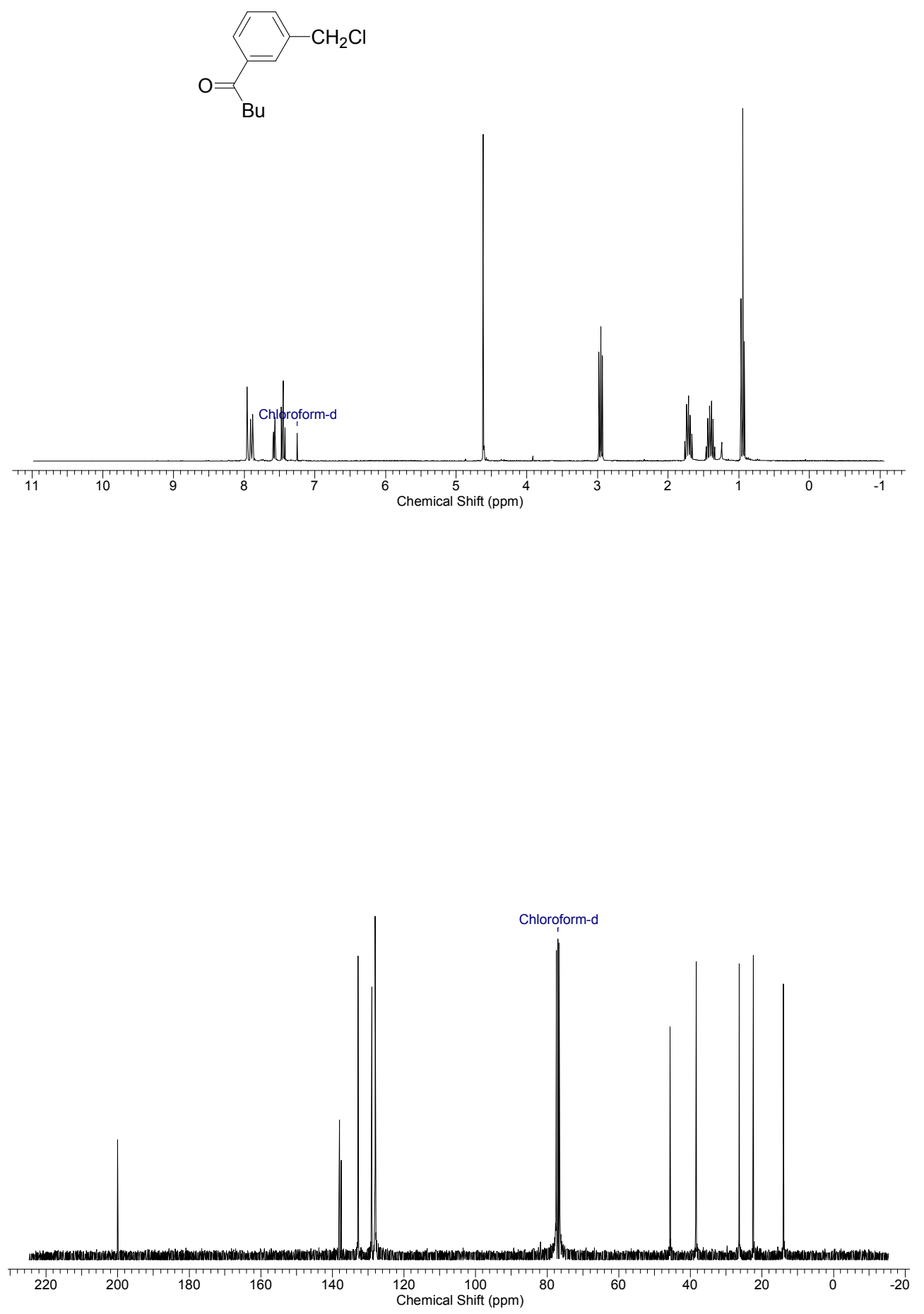
1-[3-(Chloromethyl)phenyl]propan-1-one (2h)
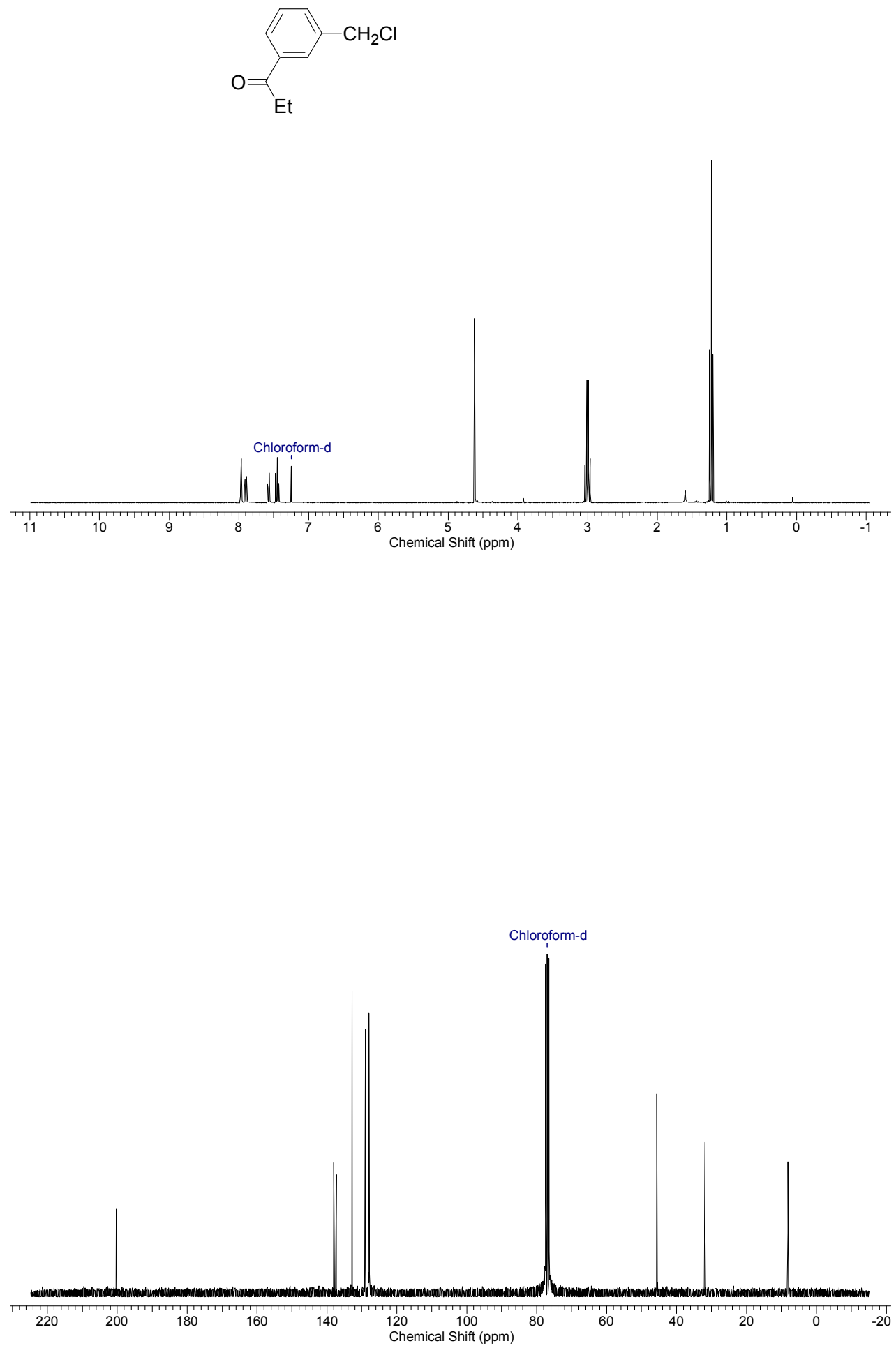
1-[3-(Chloromethyl)phenyl]propan-1-one (2i)
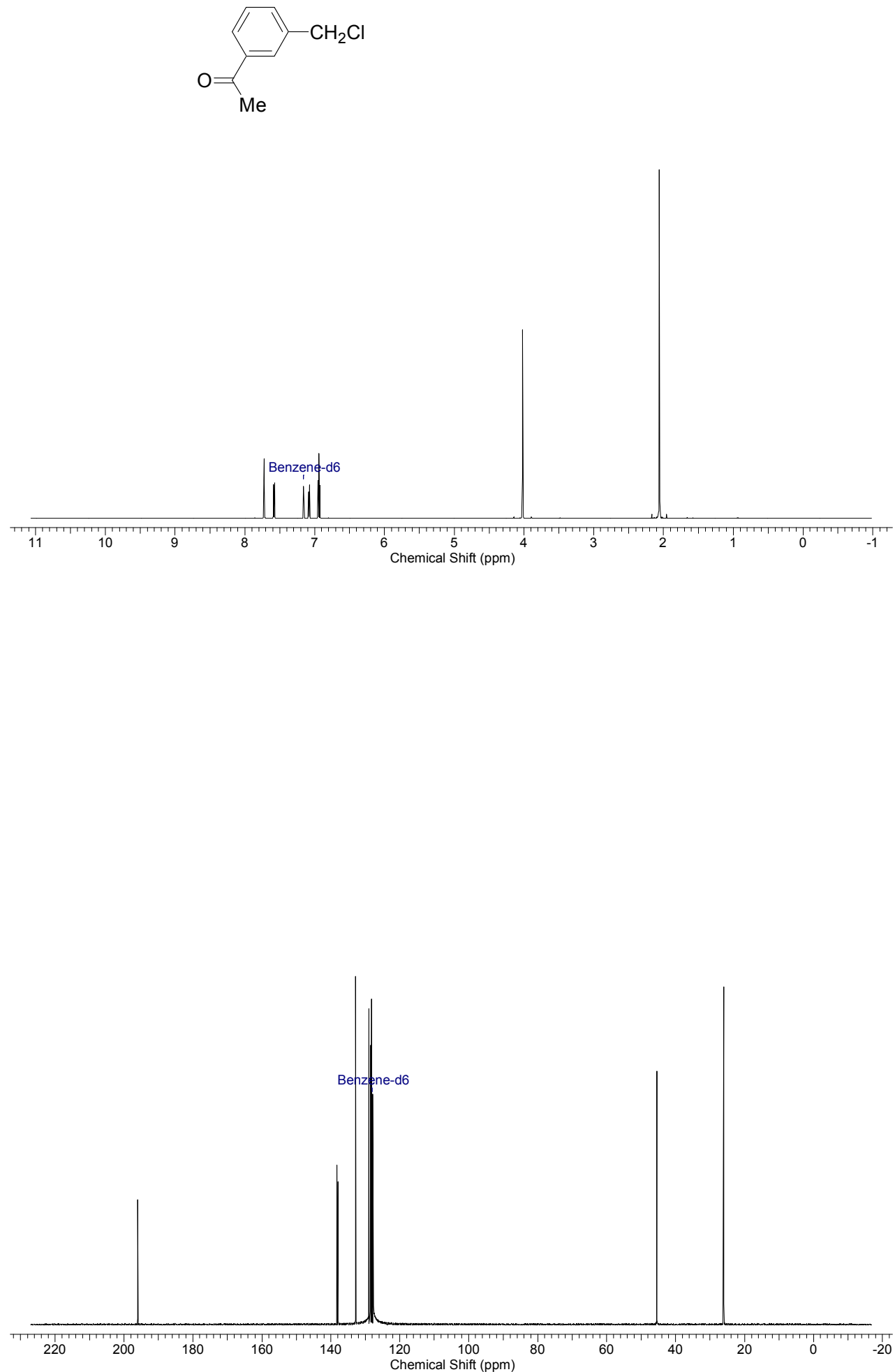
1-Chloro-2-(cyclohex-2-en-1-ylmethyl)benzene (4a)
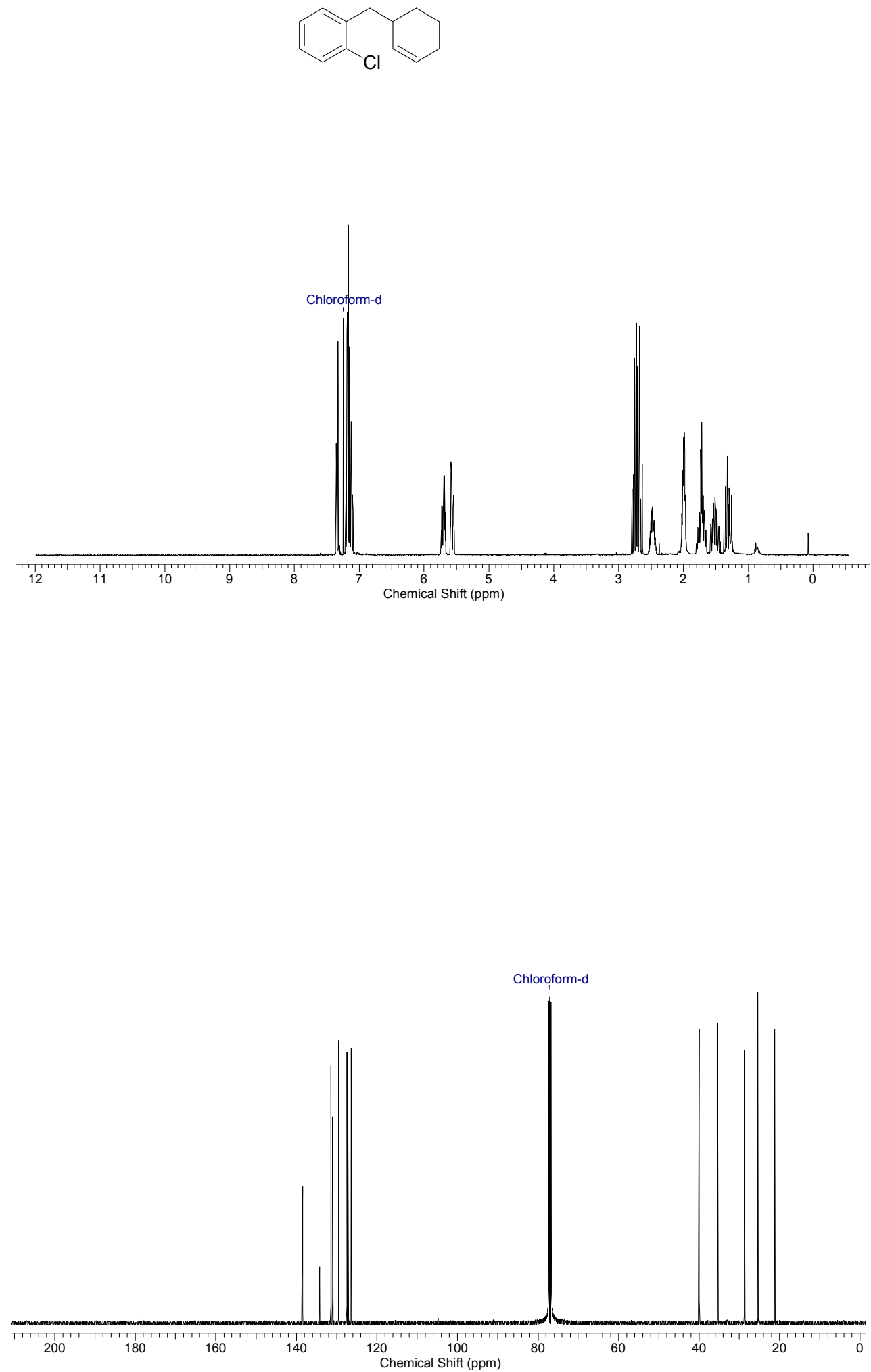
1-Chloro-2-[2-(4-nitrophenyl)ethyl]benzene (4b)
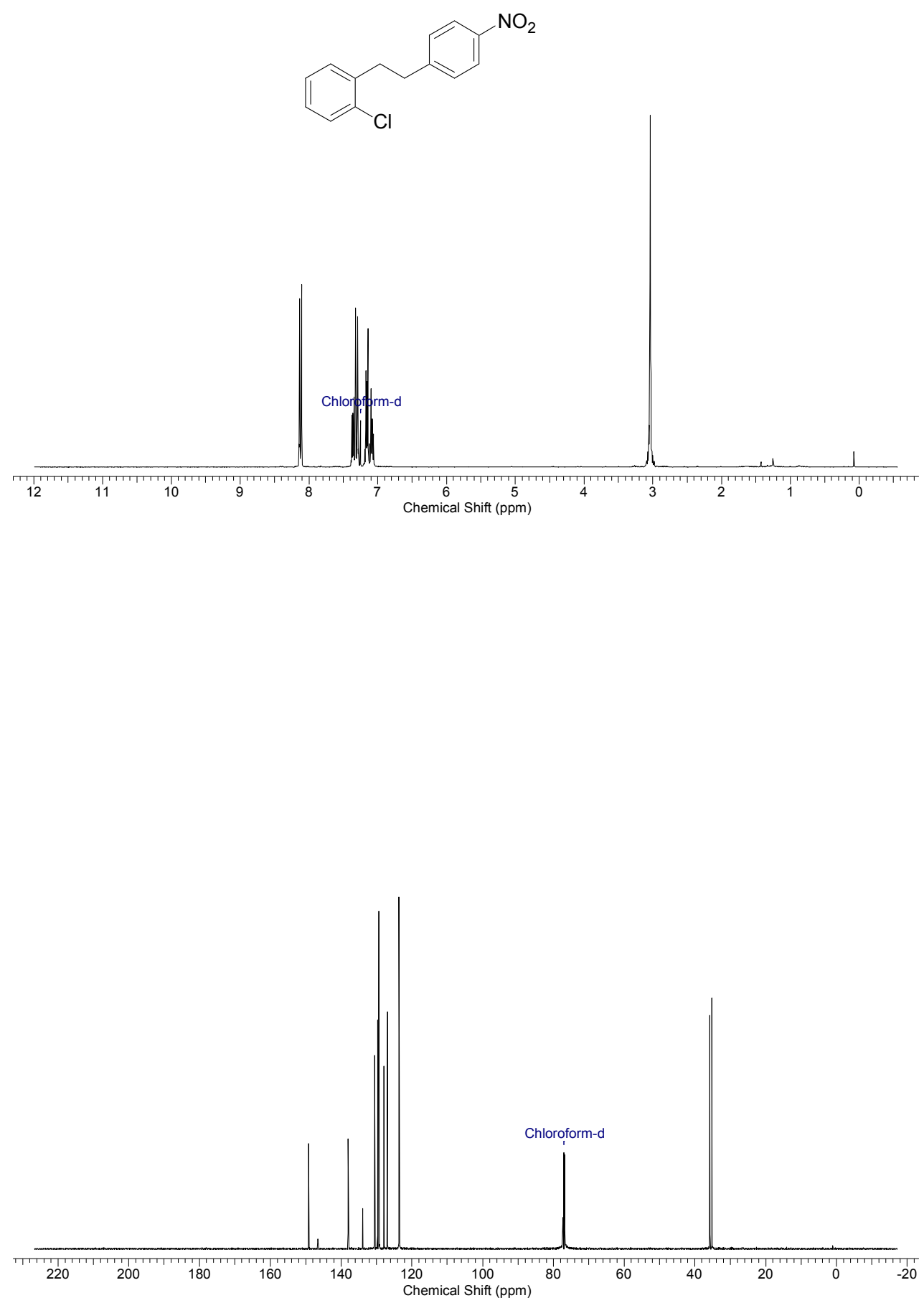
Ethyl 4-(2-chlorobenzyl)benzoate (4c)
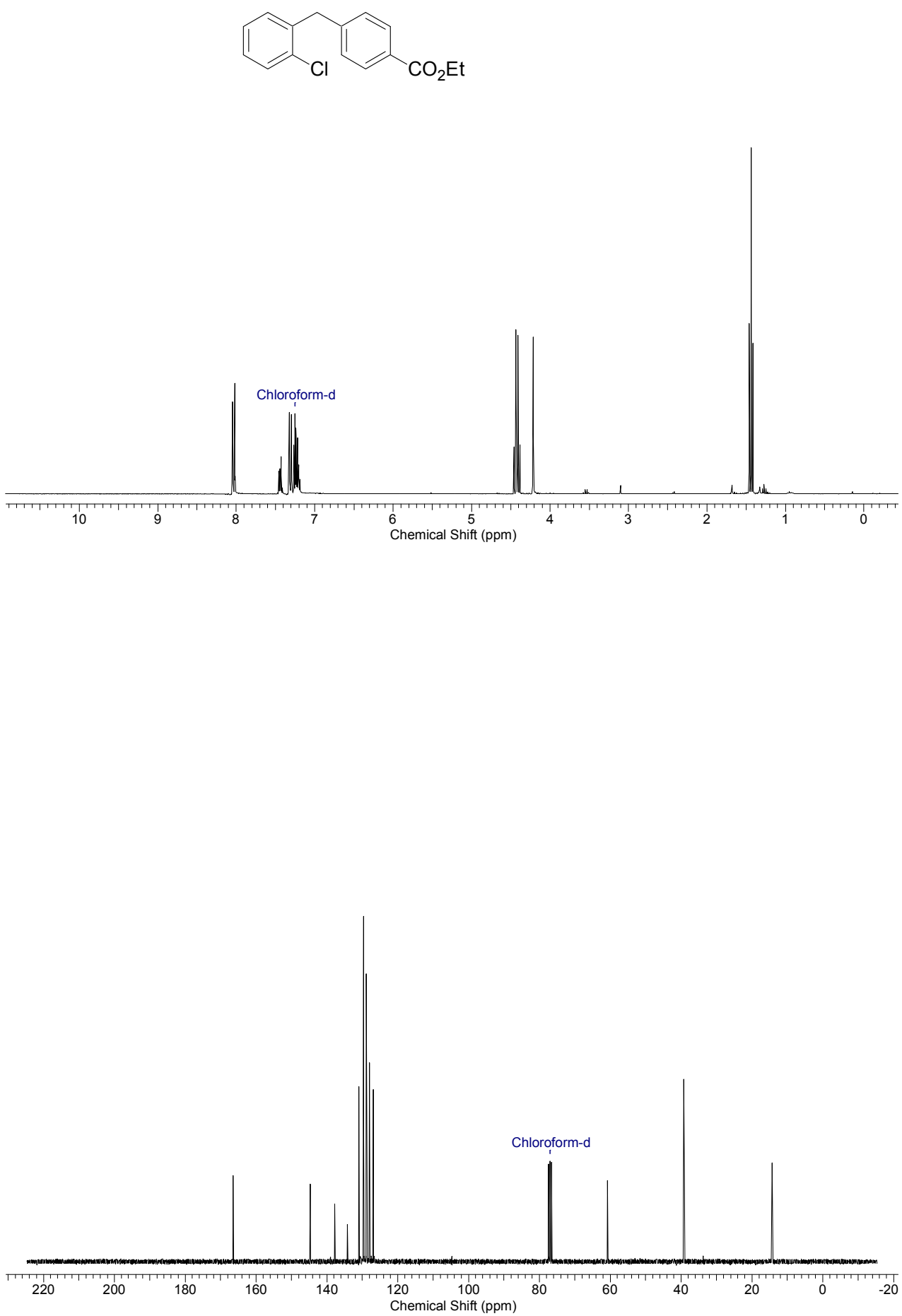

36 
3-(2-Chlorobenzyl)cyclohexanone (4d)
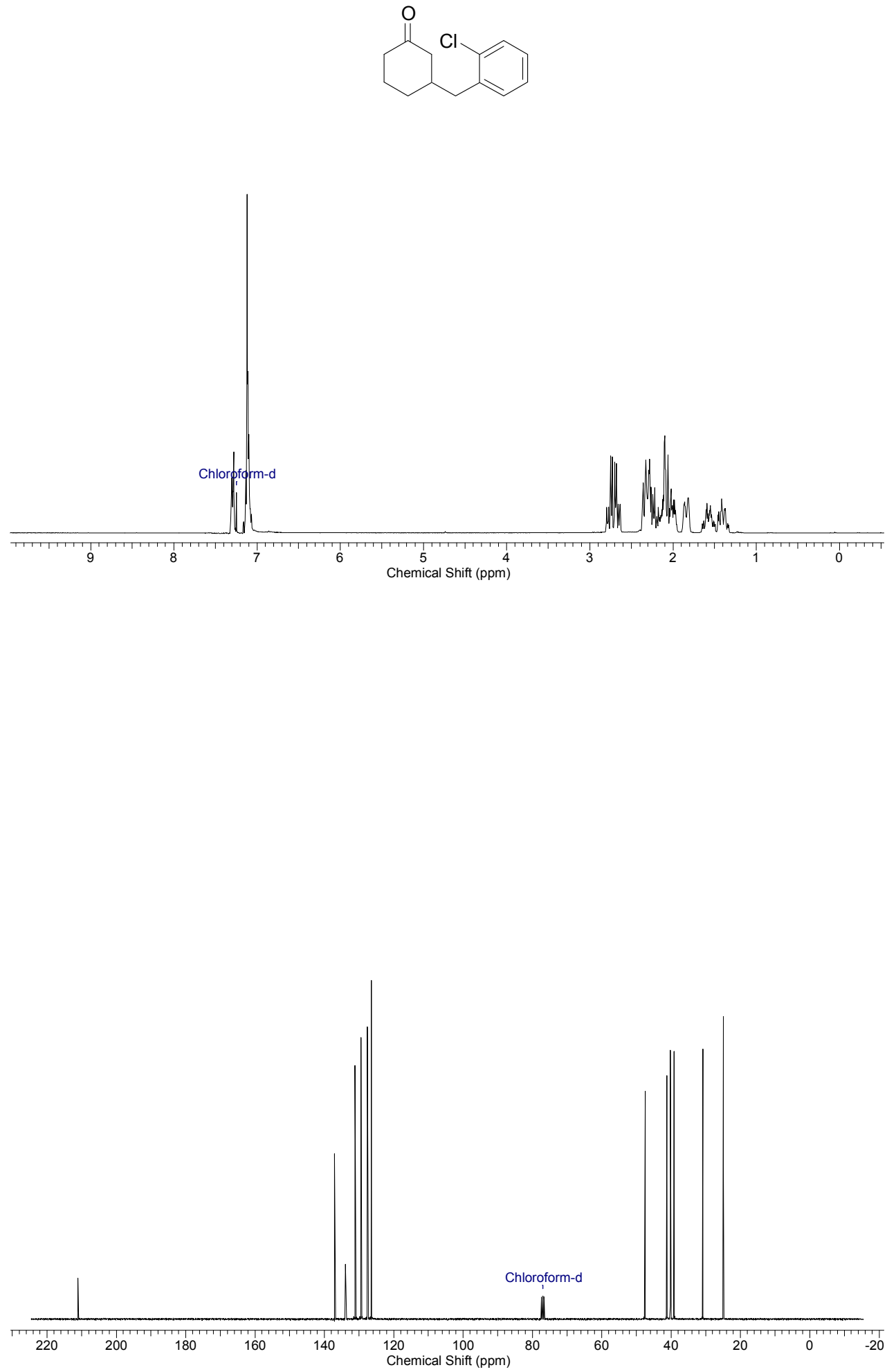
1-(4-Fluorophenyl)-3,3-dimethylbutan-2-one (4e)
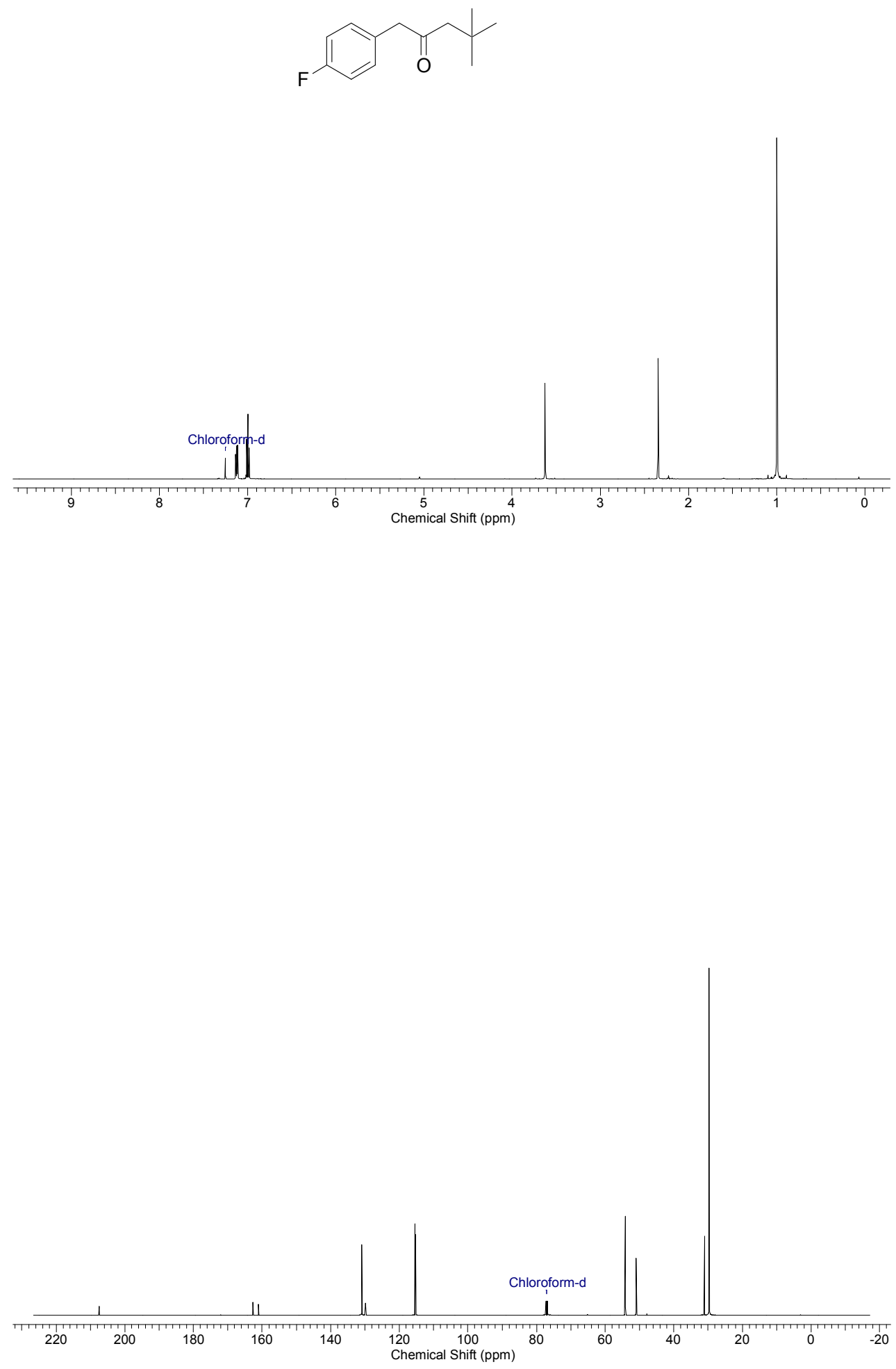
2-(3-Bromophenyl)-1-(3,4-dichlorophenyl)ethanol (4f)
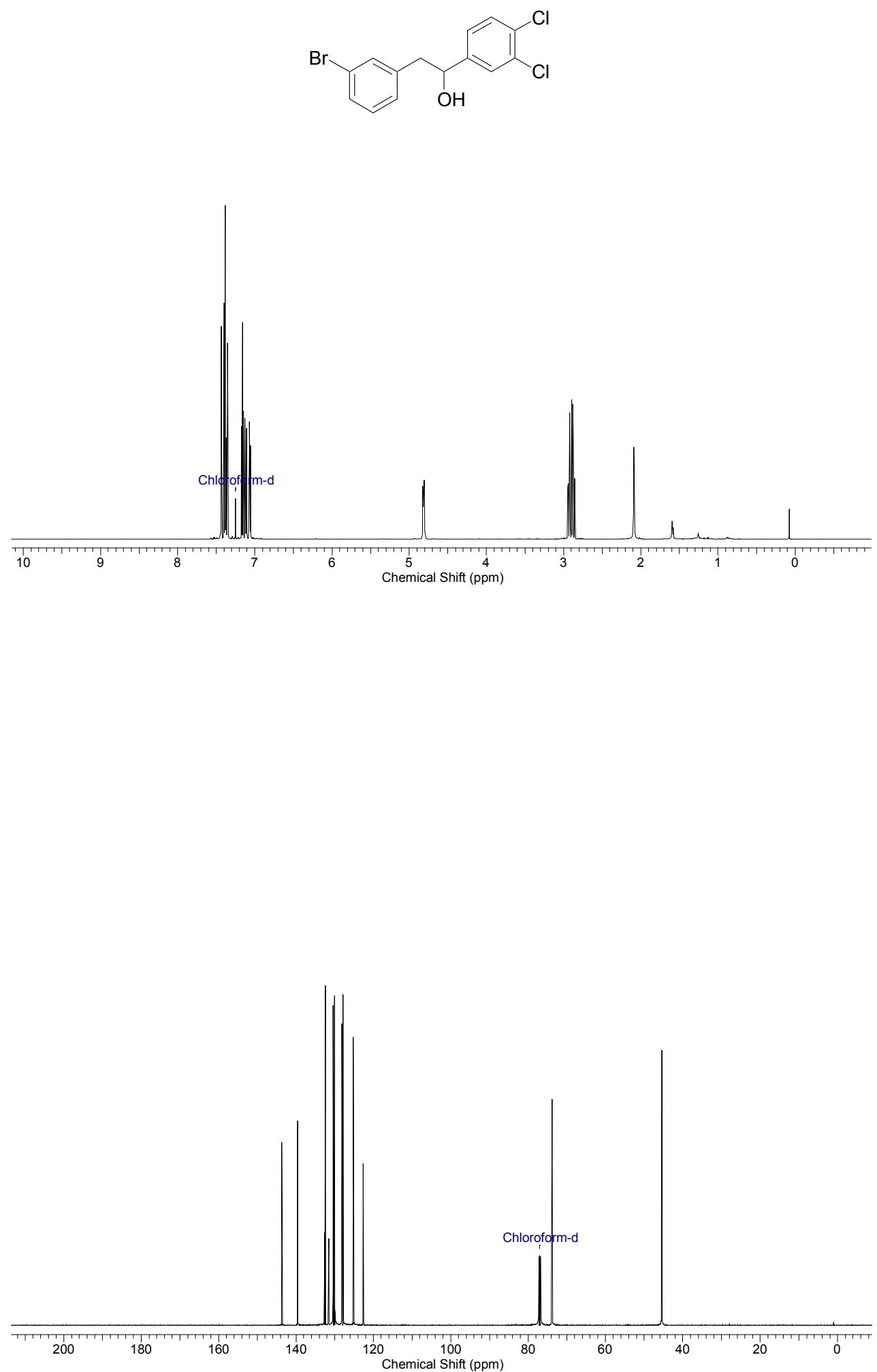
1-(3-Chlorophenyl)-2-(2-iodophenyl)ethanol (4g)
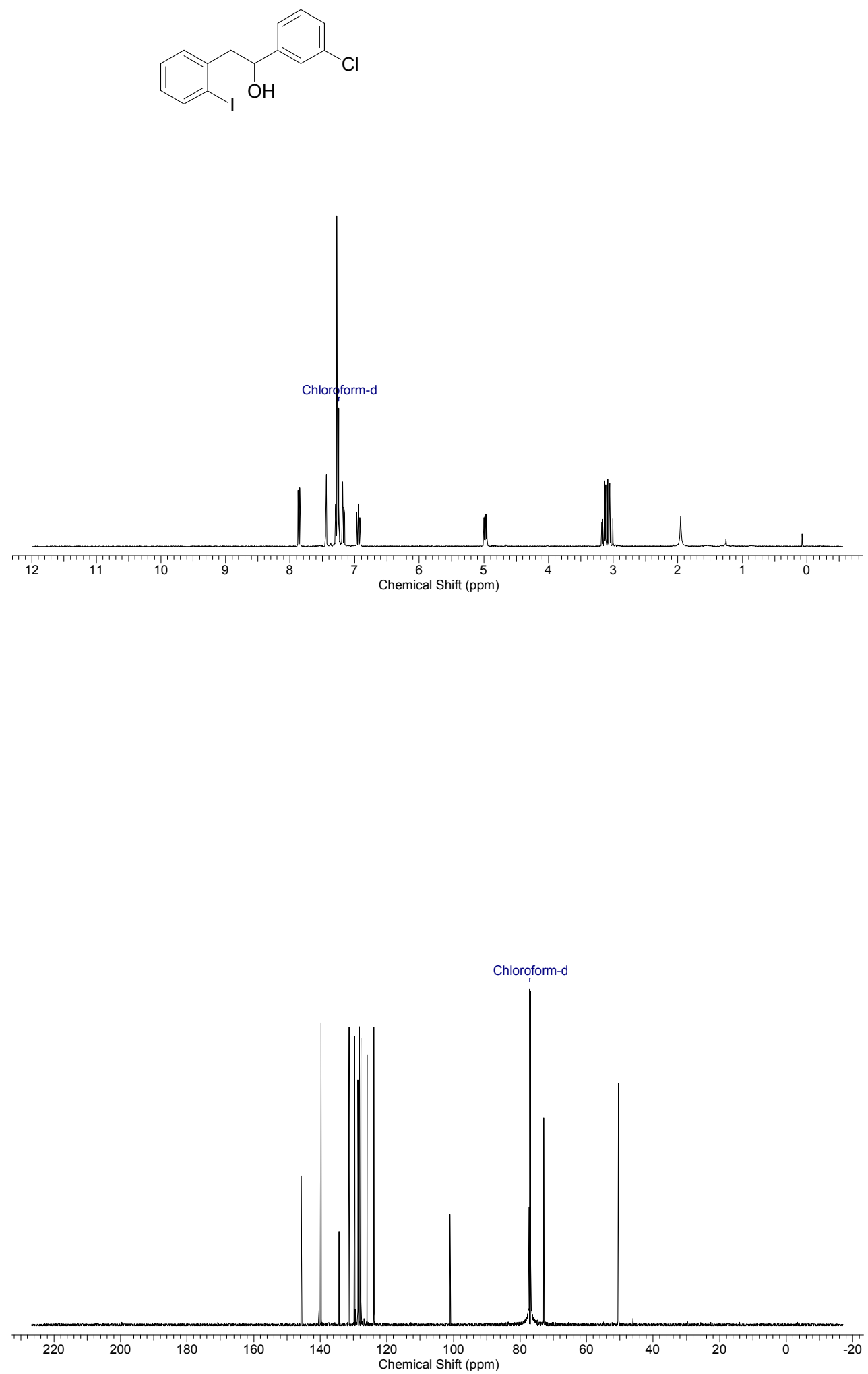
3-(2-Iodobenzyl)cyclohexanone (4h)
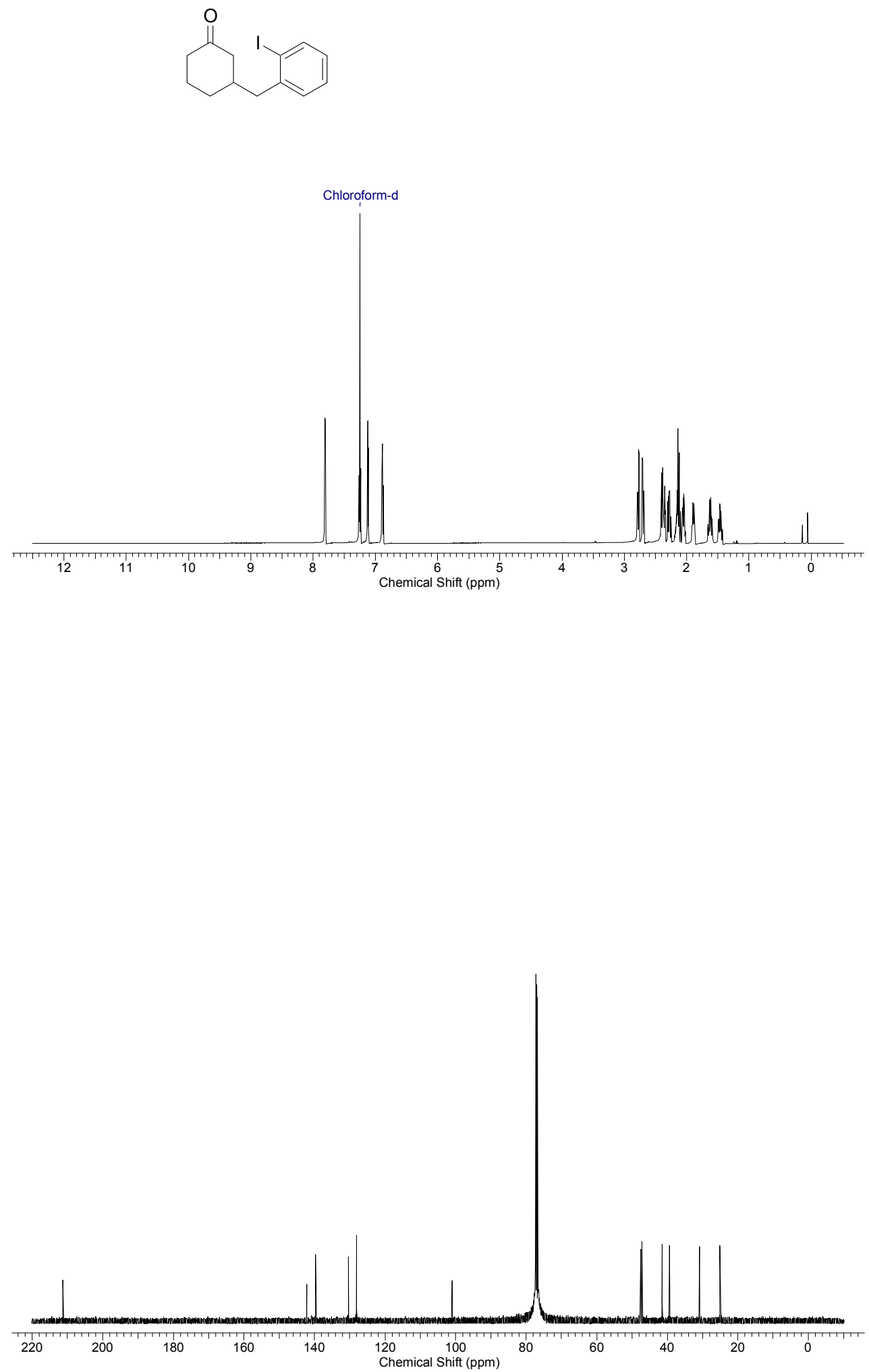
Ethyl 3-[2-(4-bromophenyl)-2-hydroxyethyl]benzoate (4i)
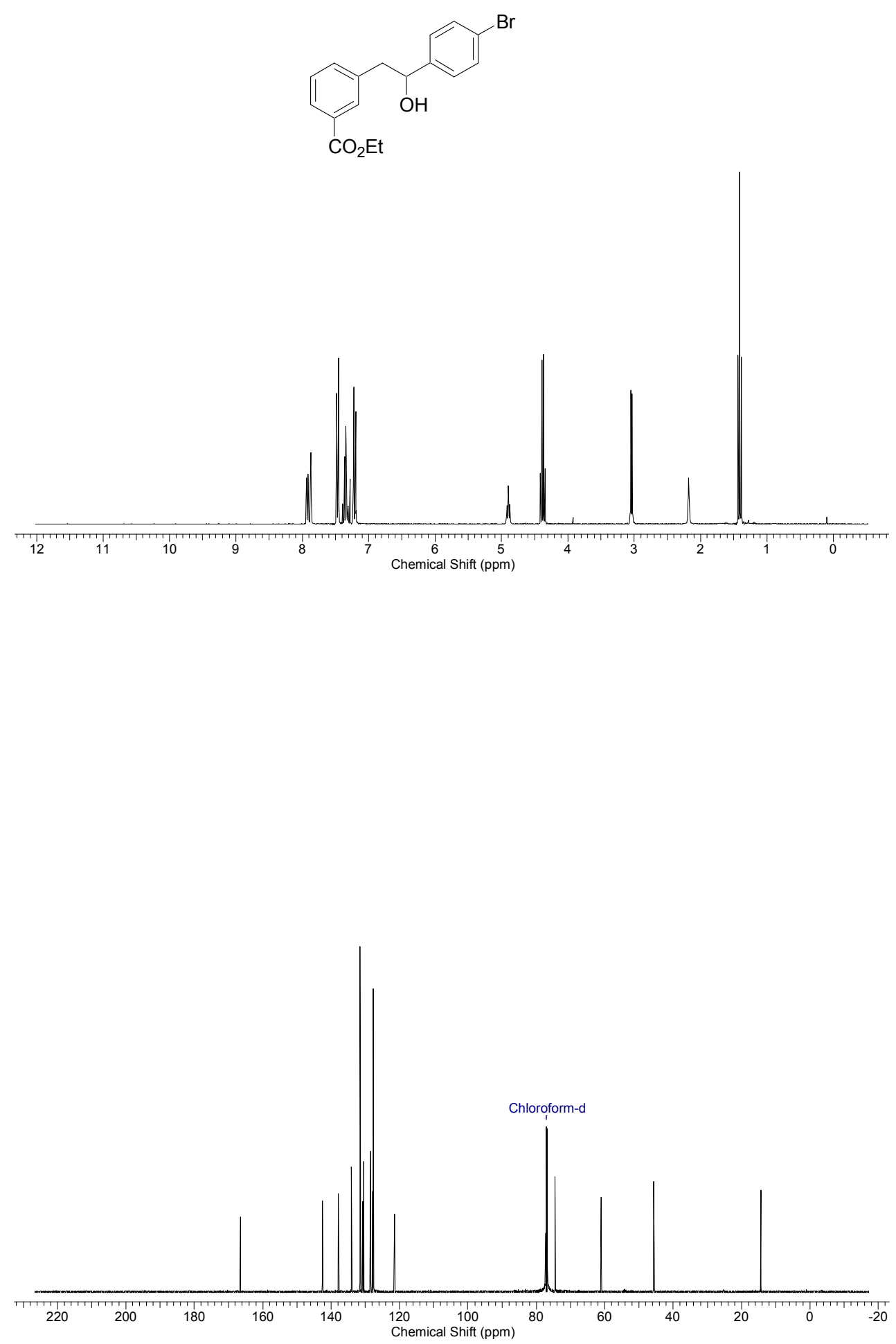
Ethyl 3-[(3-oxocyclohexyl)methyl]benzoate (4j)
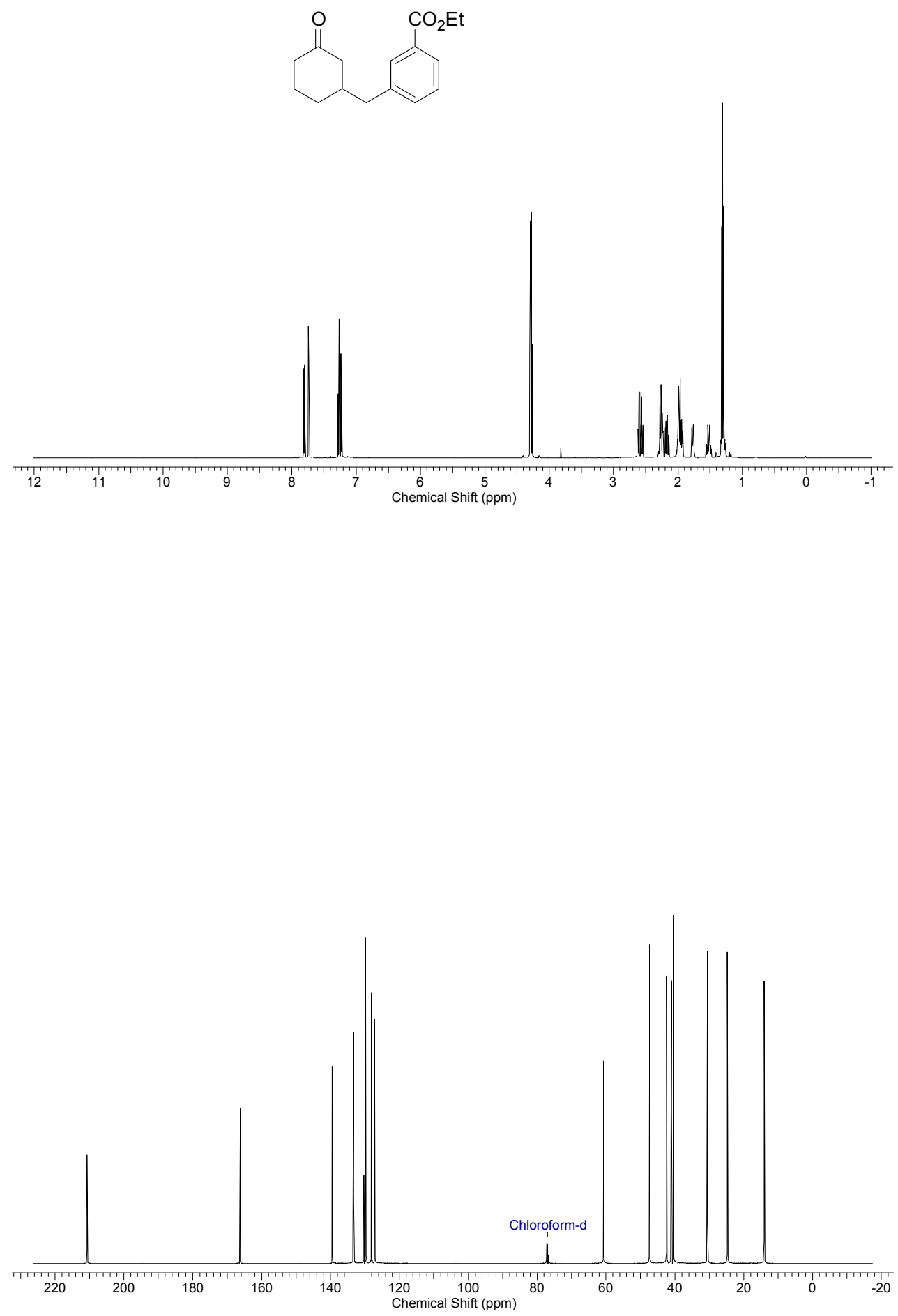
3-(3-Methoxybenzyl)benzonitrile (4k)

${ }^{\mathrm{NC}} \mathrm{OMe}$
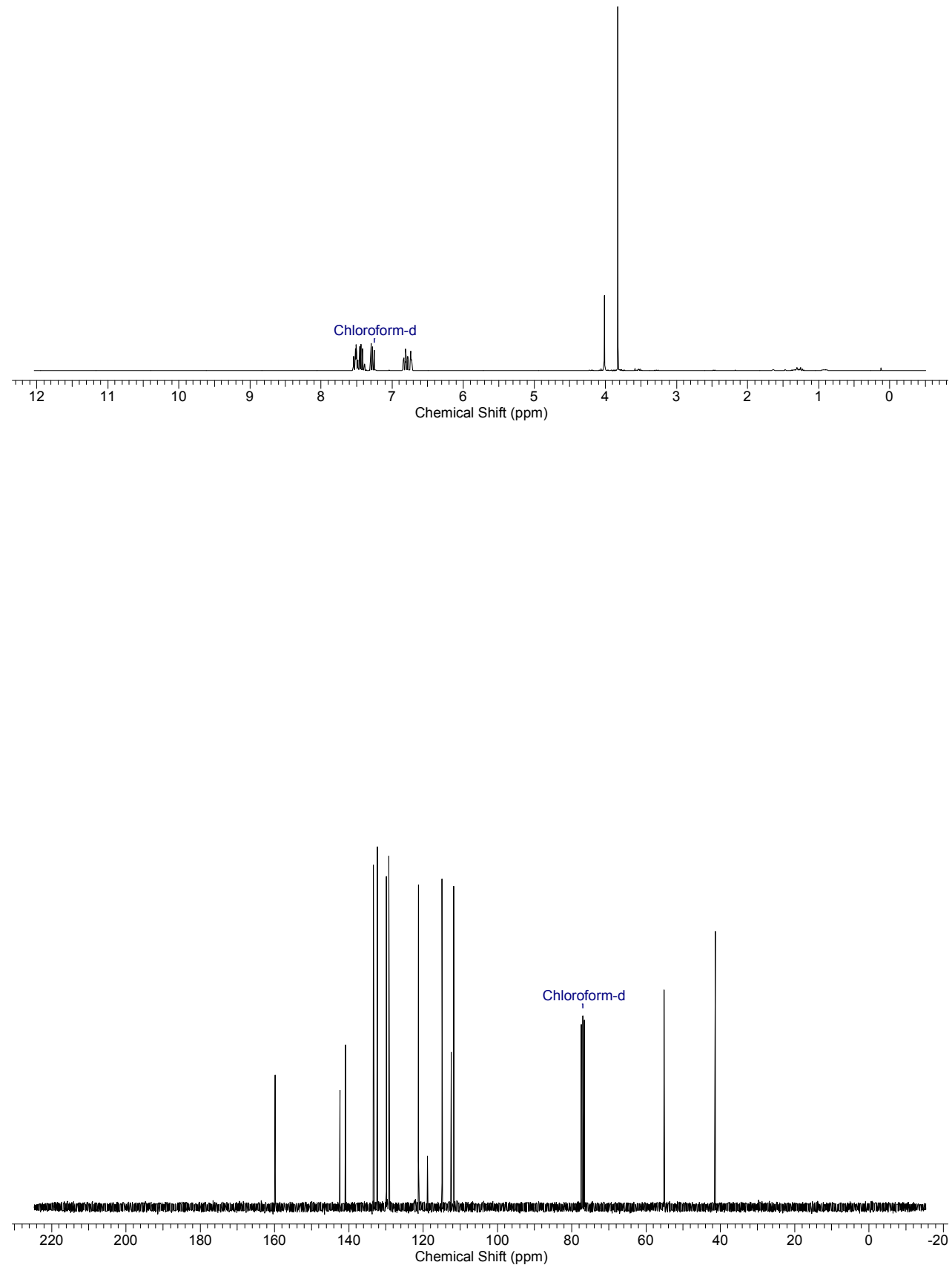
3-(3,3-Dimethyl-2-oxobutyl)benzonitrile (4l)
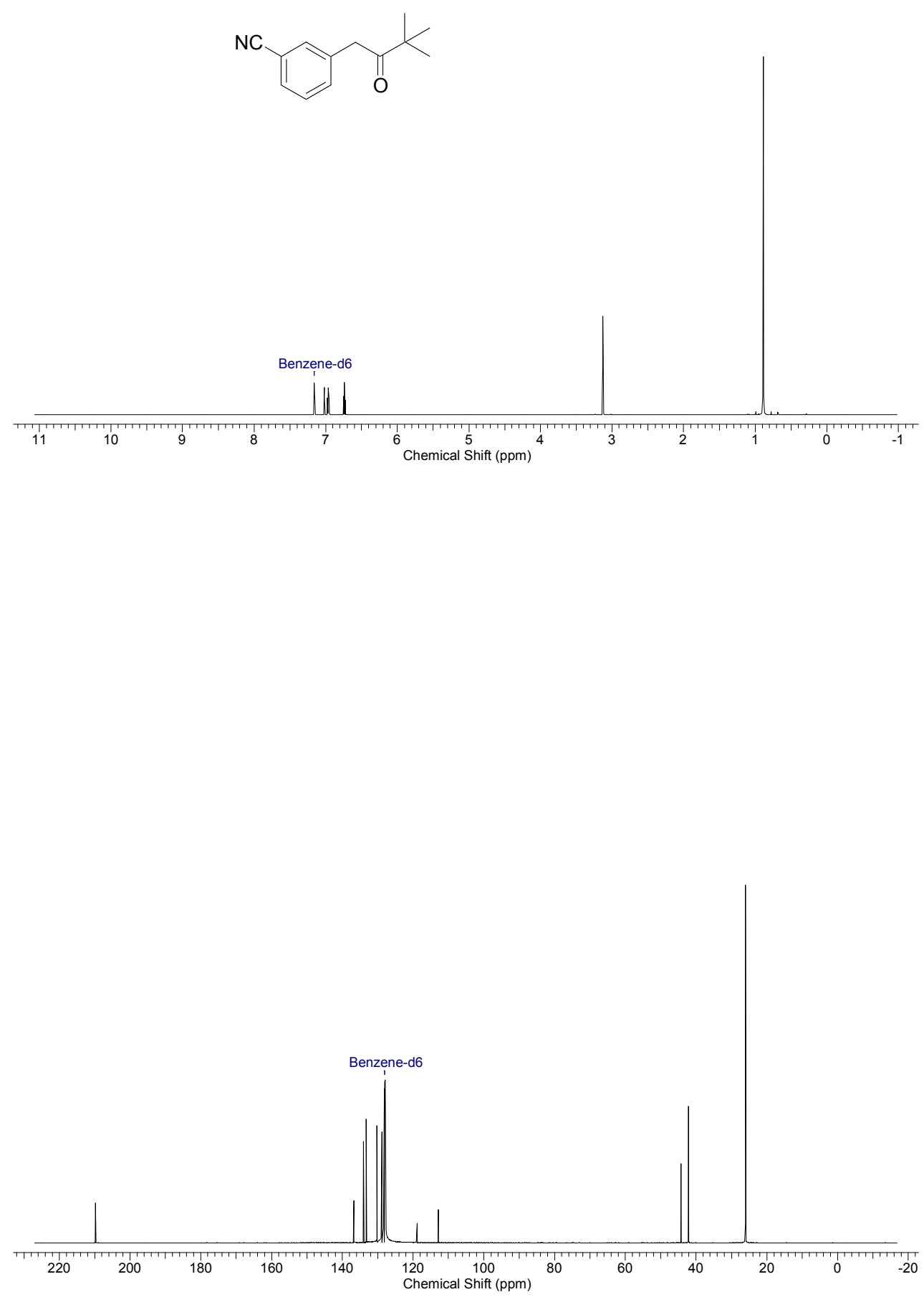
1-[3-(2-Oxo-2-phenylethyl)phenyl]pentan-1-one (4m)<smiles>O=C(Cc1cccc(C(=O)Cc2ccccc2)c1)c1ccccc1</smiles>
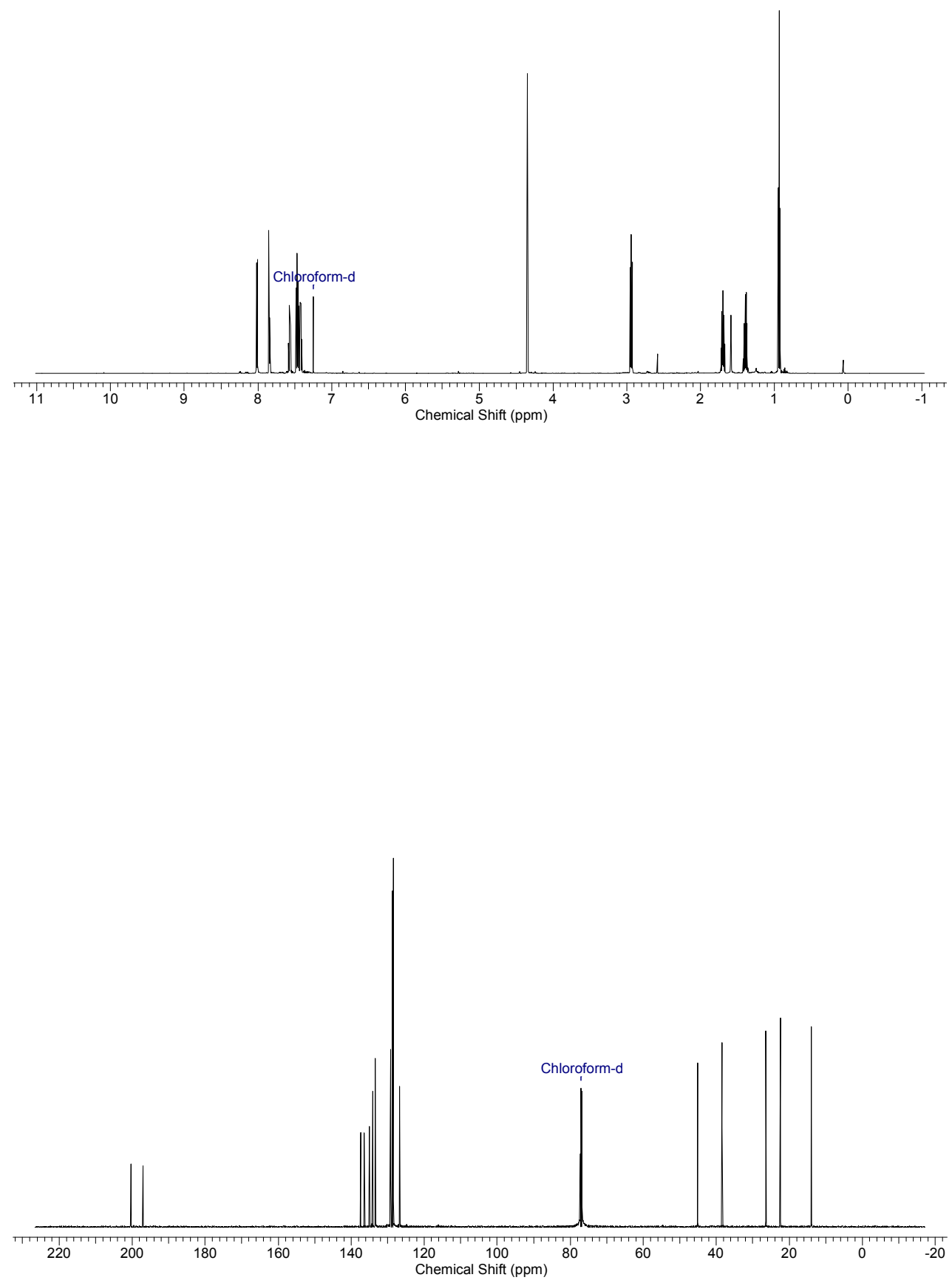
1-\{3-[2-(3,4-dichlorophenyl)-2-hydroxyethyl]phenyl\}pentan-1-one (4n)<smiles>O=C(Br)c1cccc(CC(O)c2ccc(Cl)c(Cl)c2)c1</smiles>
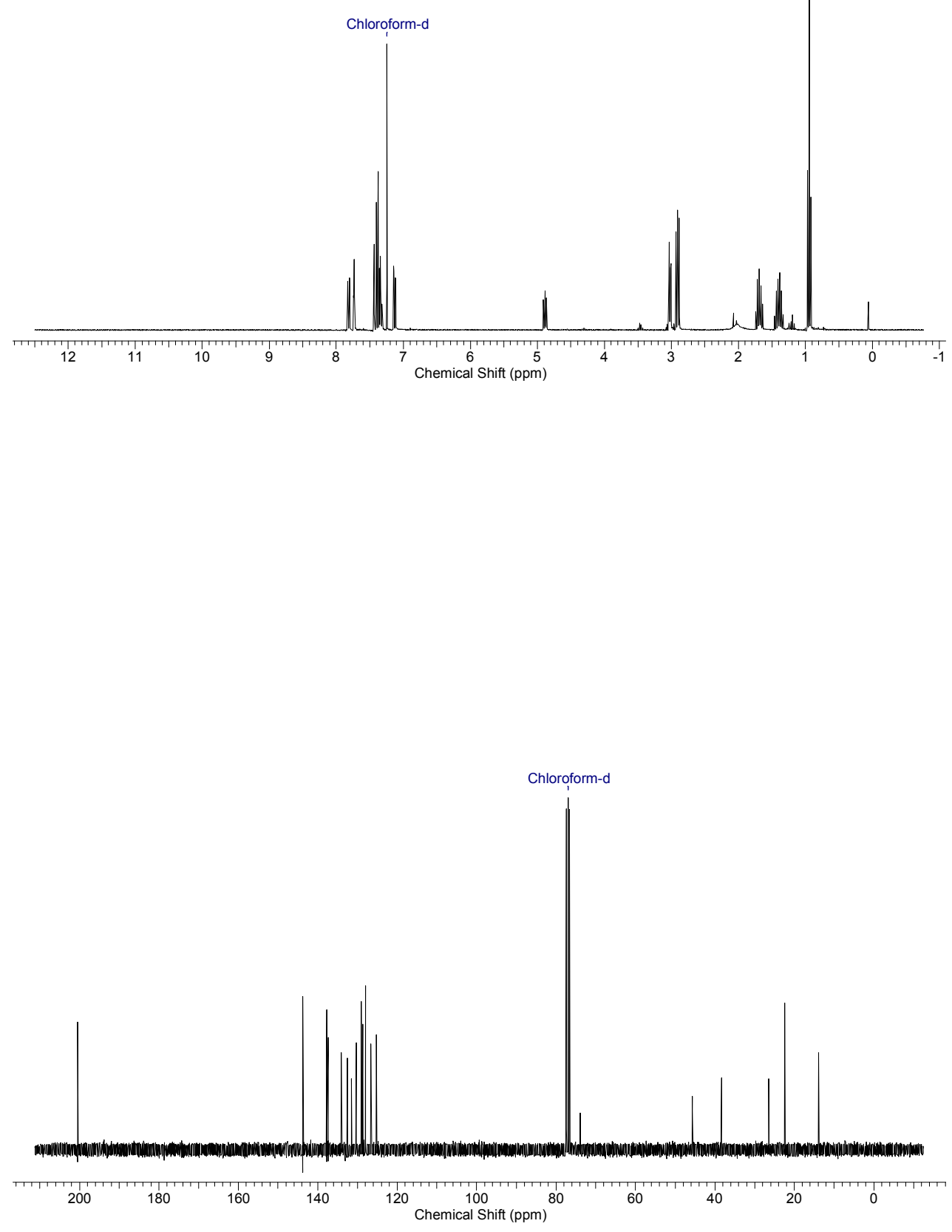
Ethyl 2-[2-(3-propionylphenyl)ethyl]acrylate (40)
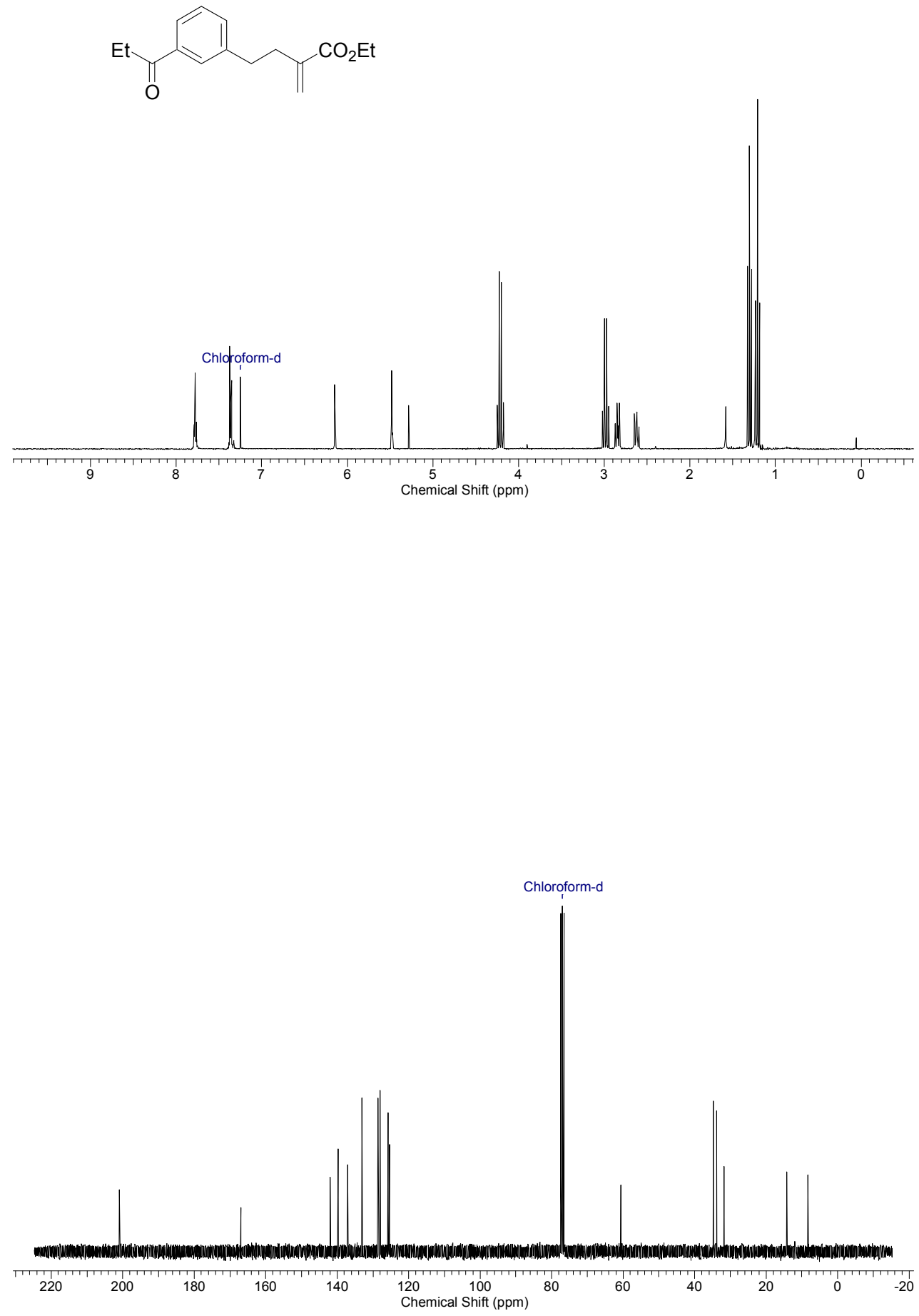
1-(3-Acetylphenyl)-4,4-dimethylpentan-2-one (4p)
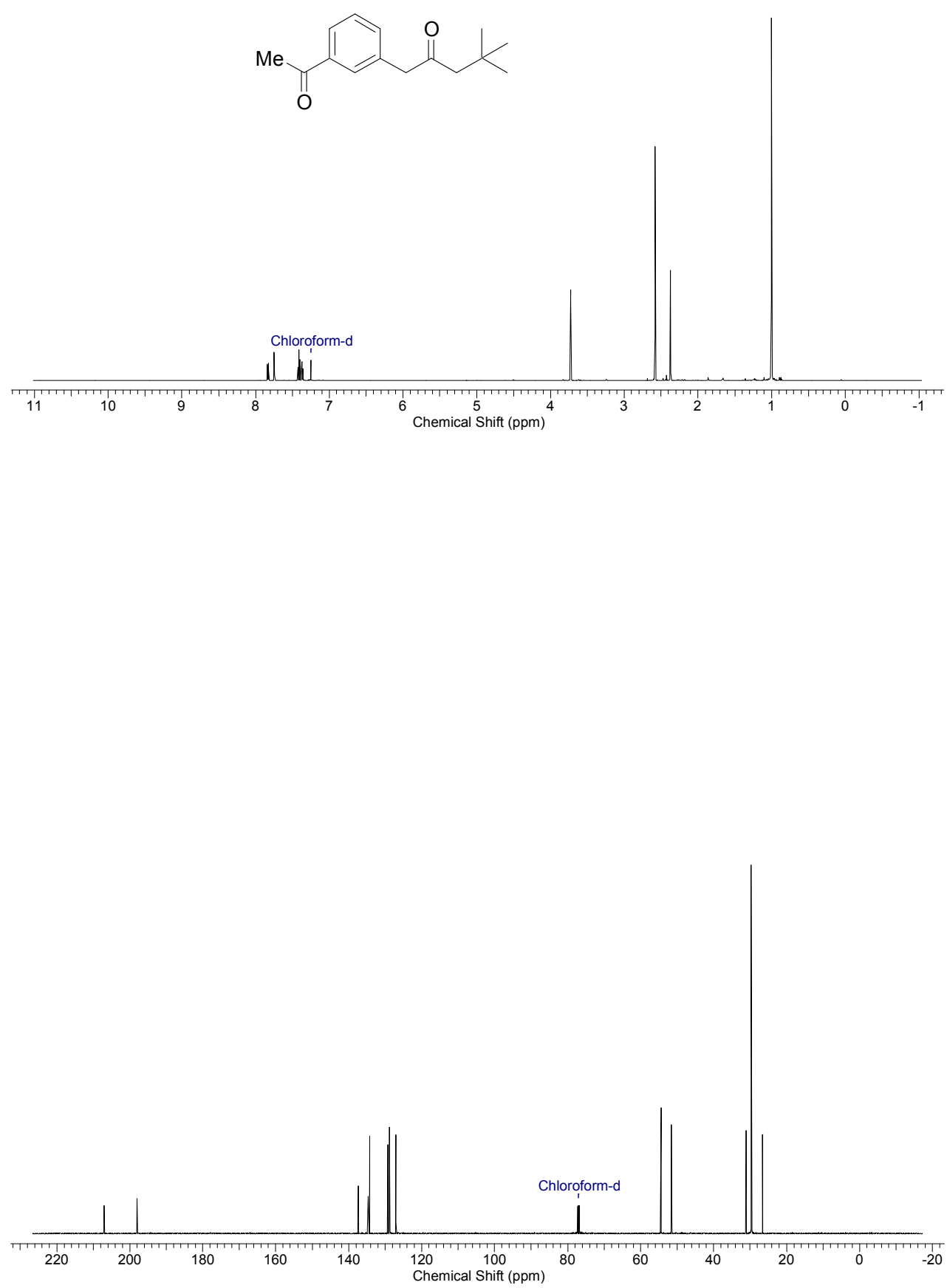
5,5-Dimethyl-2-phenylhexan-3-one (4q)
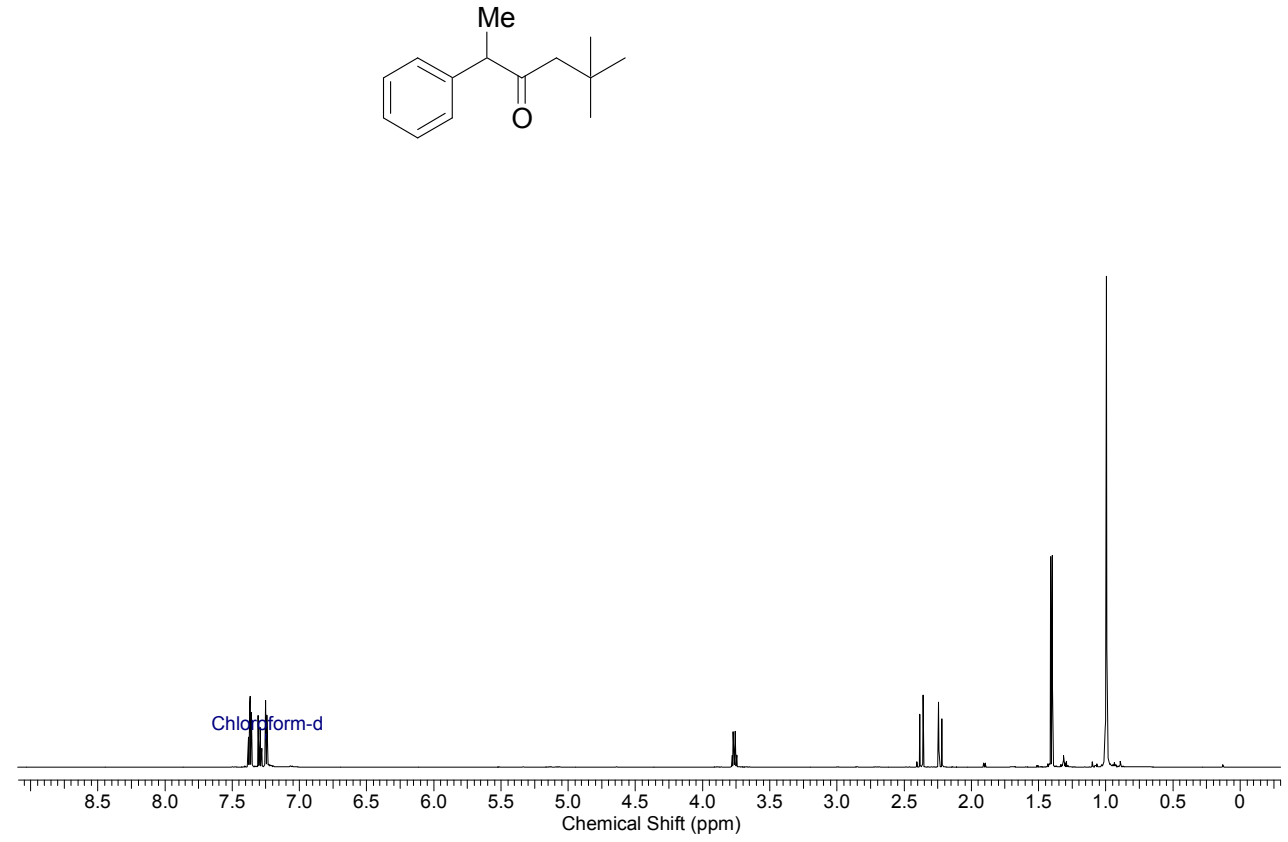
Ethyl 2-[2-(3,4,5-trimethoxyphenyl)ethyl]acrylate (4r)
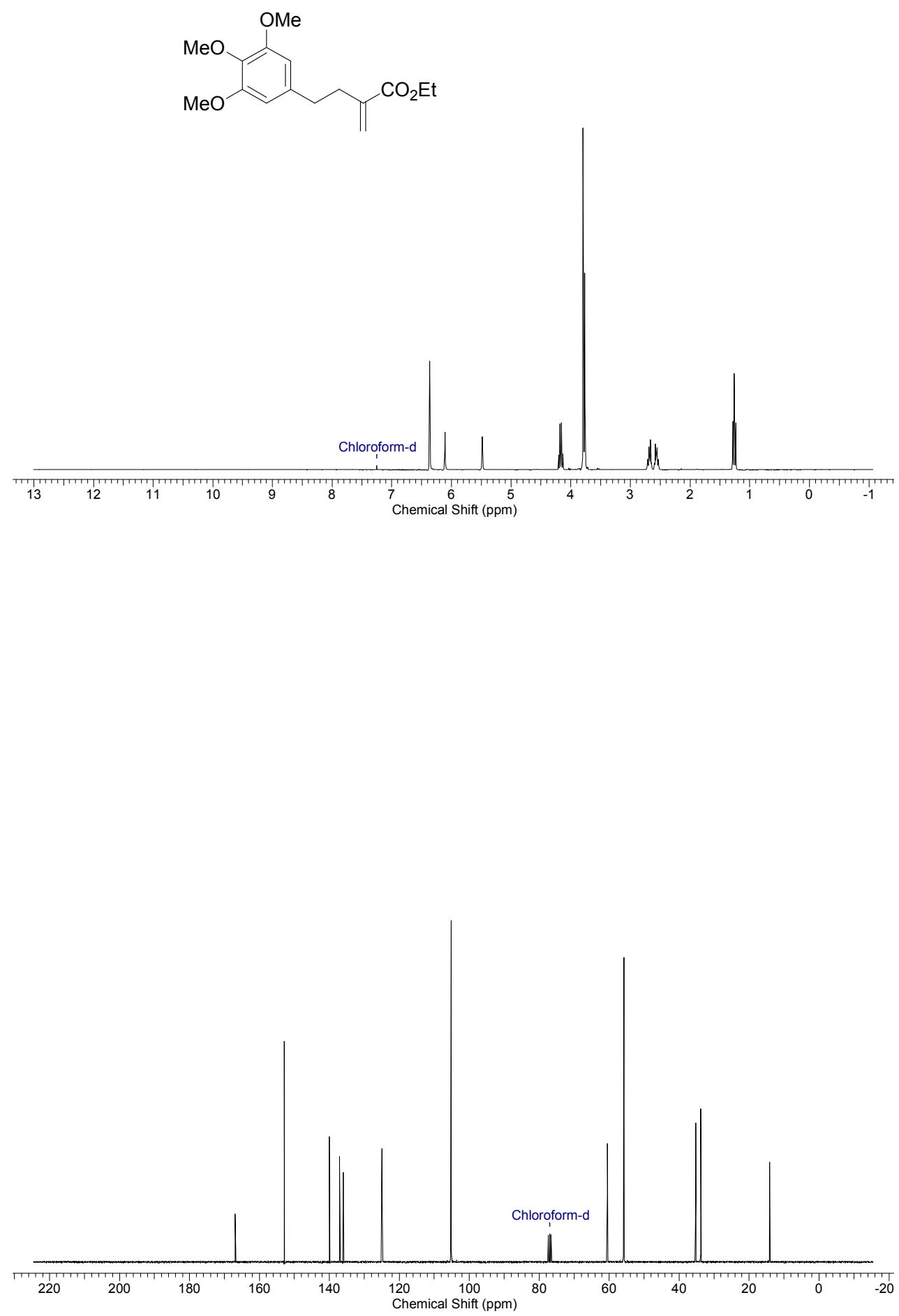
1-(6-Chloro-1,3-benzodioxol-5-yl)-4,4-dimethylpentan-2-one (4s)
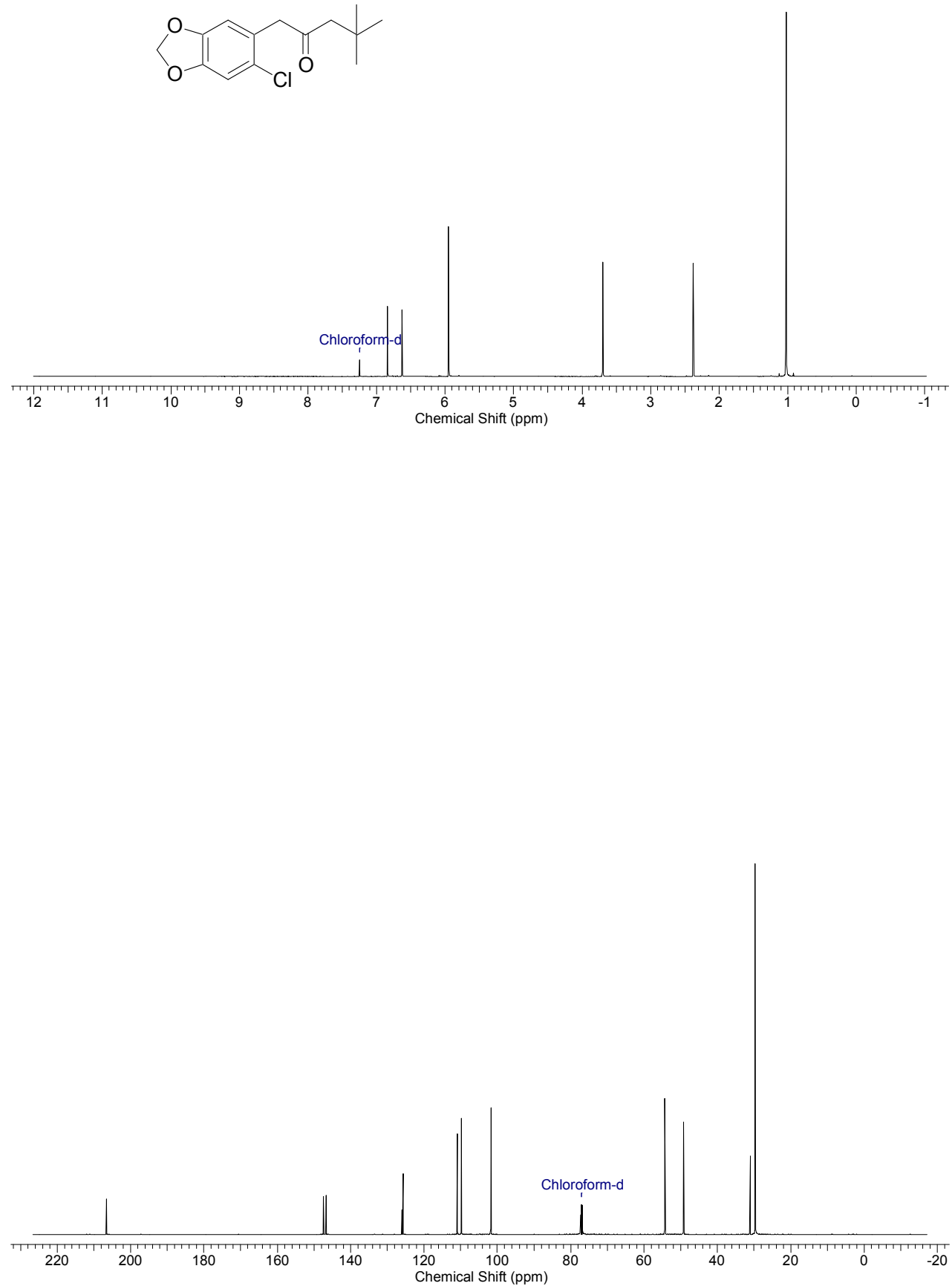
Ethyl (2-chlorophenyl)acetate (5)
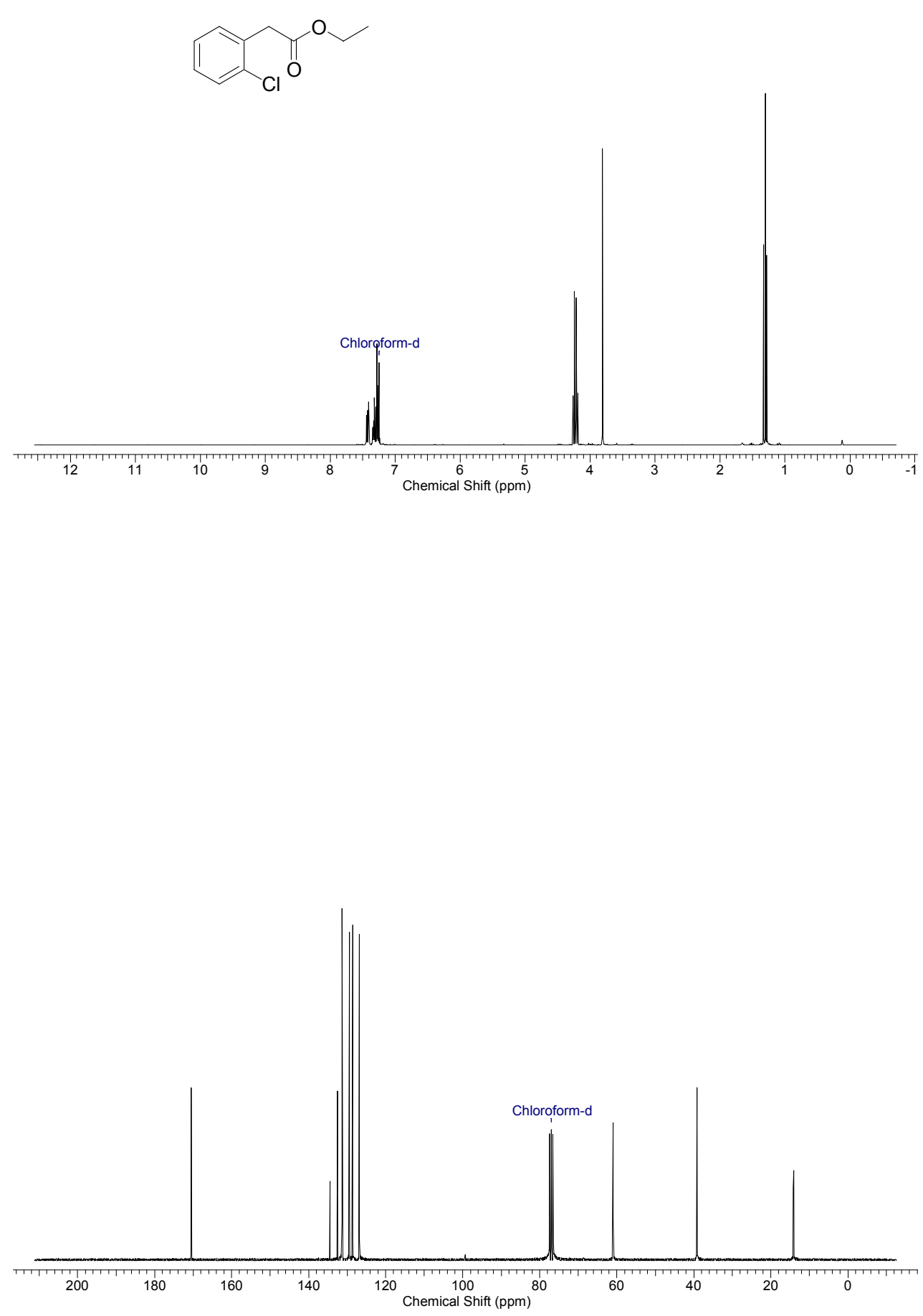
$N$-[(1E)-(3,4-dimethoxyphenyl)methylene]-2,2-dimethoxyethanamine (9)
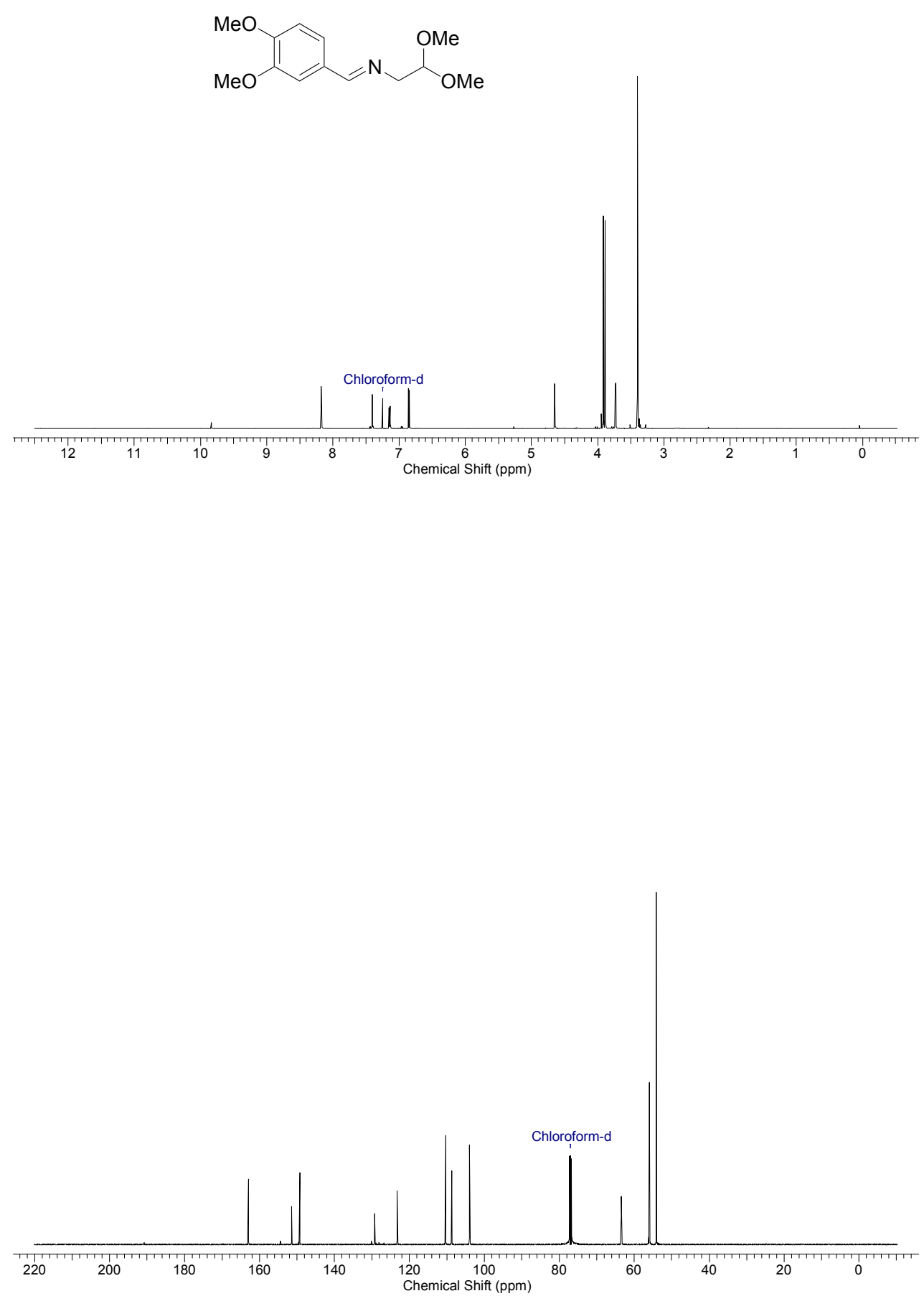


\section{(3,4-Dimethoxybenzyl)(2,2-dimethoxyethyl)amine (10)}
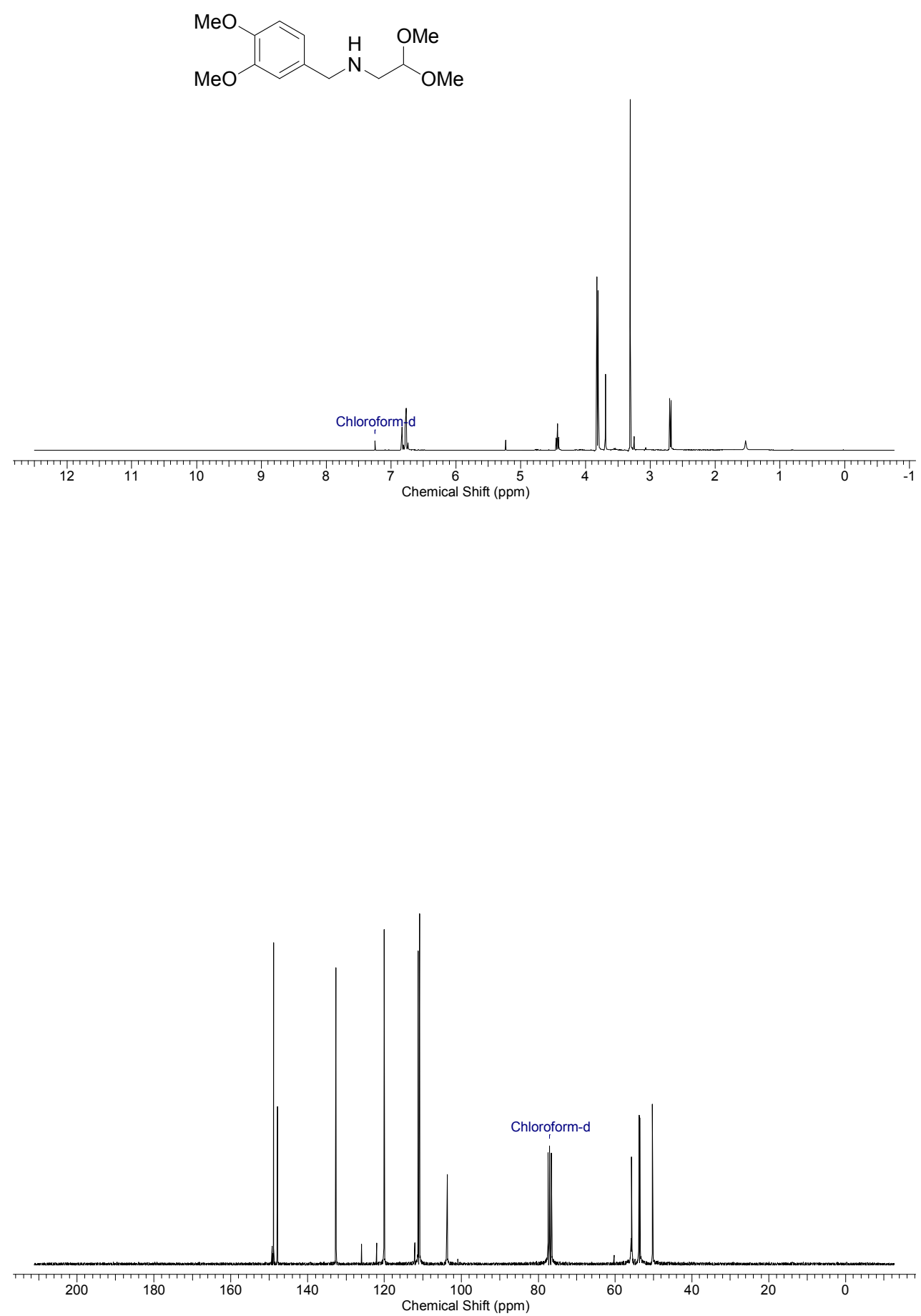

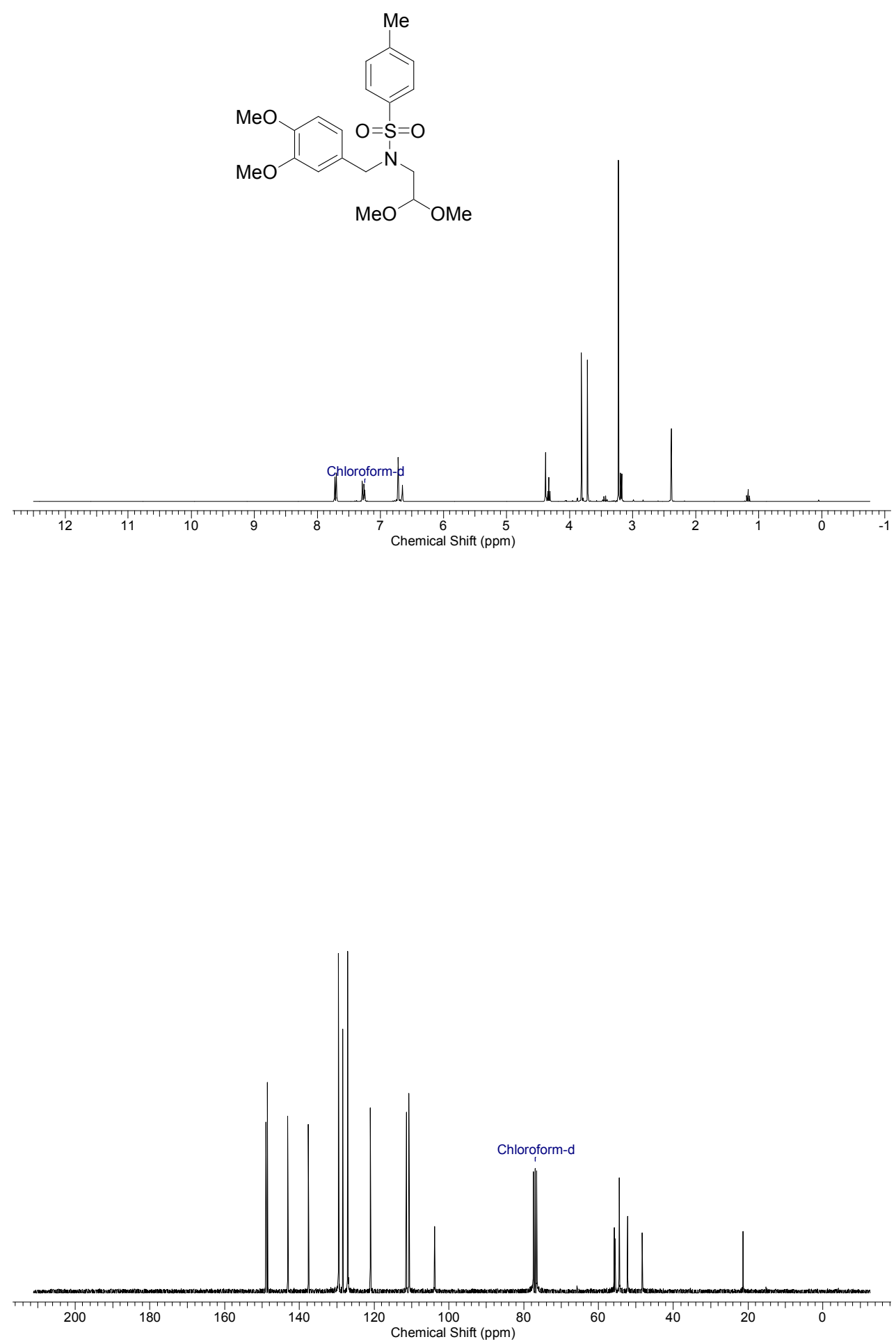
6,7-Dimethoxyisoquinoline (7)
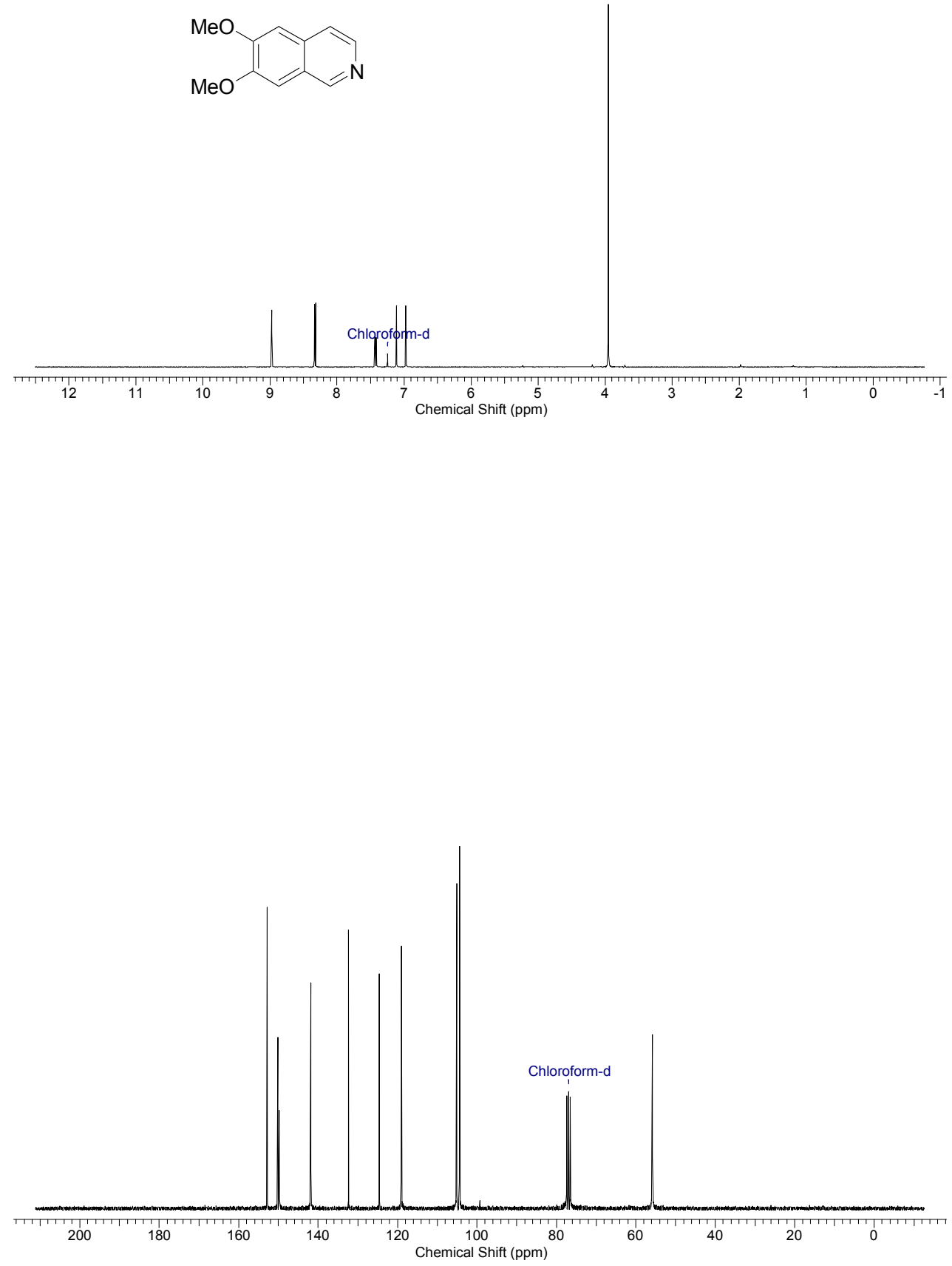
1-Iodo-6,7-dimethoxyisoquinoline (8) 474
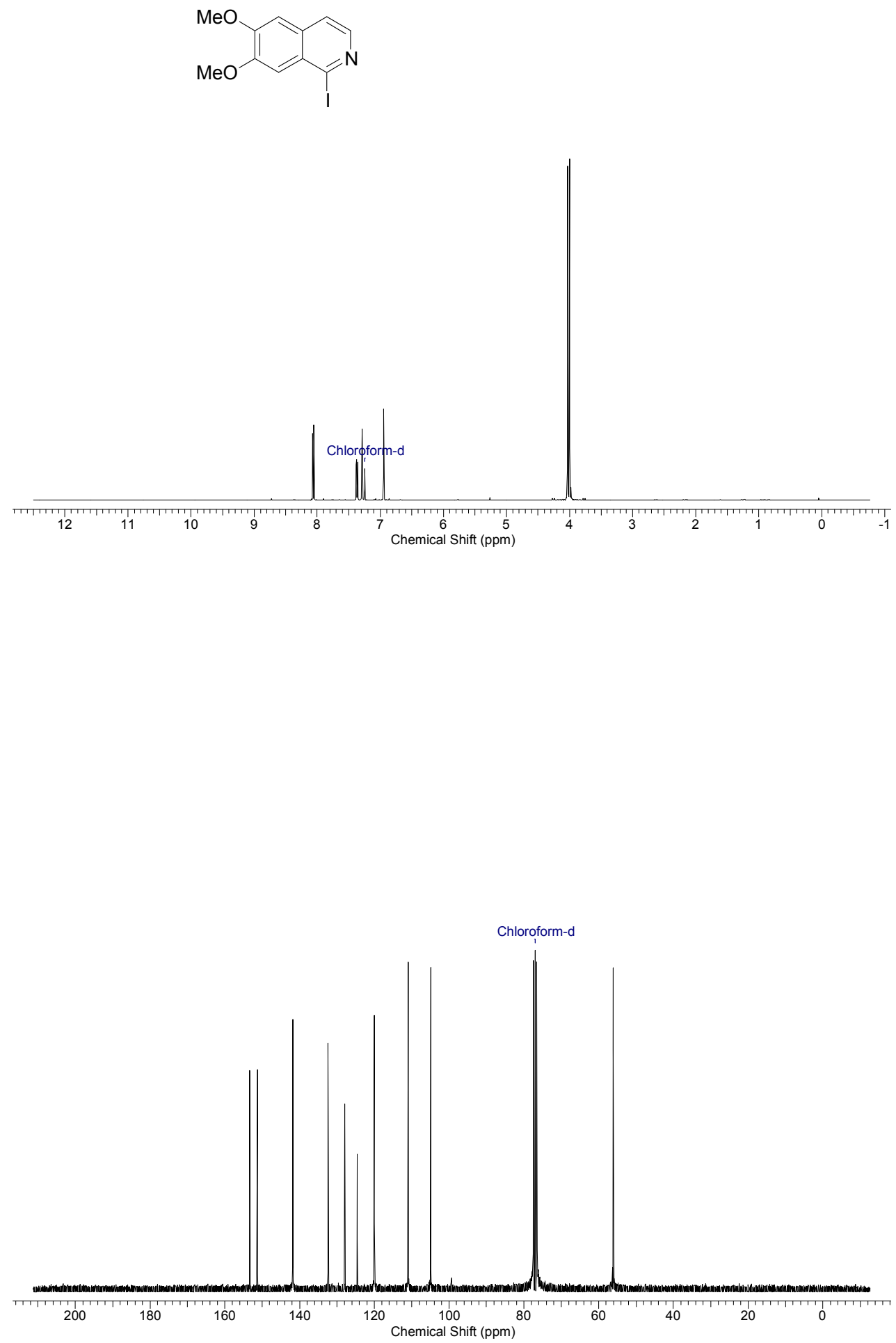
4-(Chloromethyl)-1,2-dimethoxybenzene (2m)
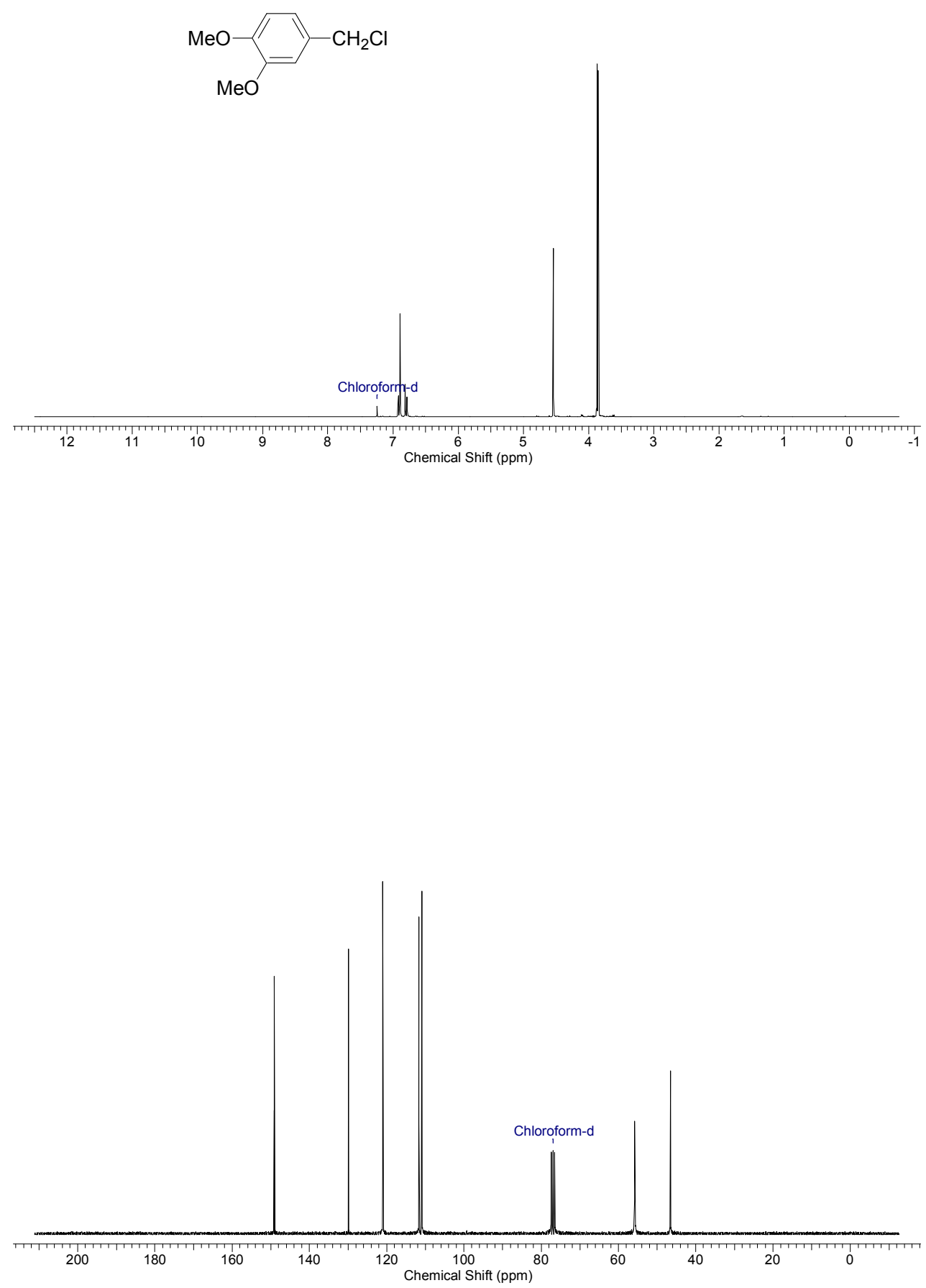
Papaverine (6)
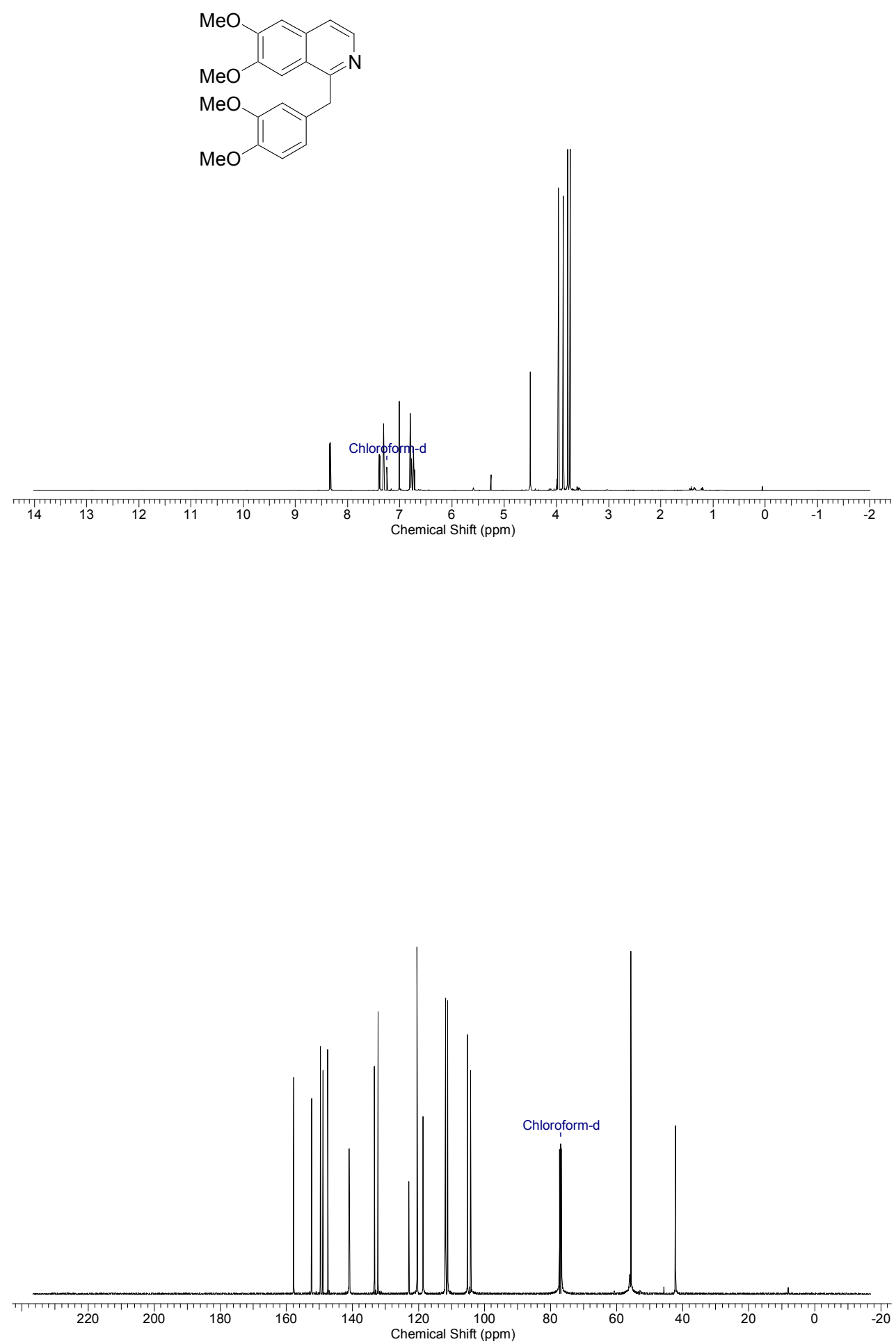